NIST

PUBLICATIONS

\title{
Detection of Incipient Defects \\ in Cables by \\ Partial Discharge Signal Analysis
}

F. D. Martzloff
E. Simmon
J. P. Steiner
R. J. Van Brunt

U.S. DEPARTMENT OF COMMERCE

Technology Administration

National Institute of Standards

and Technology

Electronics and Electrical Engineering Laboratory

Electricity Division

Gaithersburg, MD 20899

Prepared for:

United States Nuclear Regulatory

Commission

$-Q C$ 



\section{Detection of Incipient Defects} in Cables by

\author{
F. D. Martzloff \\ E. Simmon \\ J. P. Steiner \\ R. J. Van Brunt
}

U.S. DEPARTMENT OF COMMERCE Technology Administration National Institute of Standards and Technology

Electronics and Electrical Engineering Laboratory

Electricity Division

Gaithersburg, MD 20899

Prepared for:

United States Nuclear Regulatory

Commission

July 1992

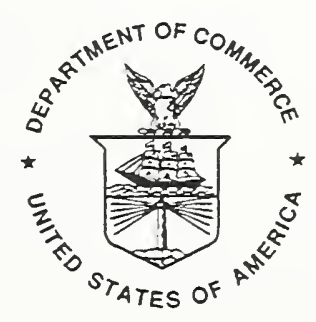

U.S. DEPARTMENT OF COMMERCE Barbara Hackman Franklin, Secretary

TECHNOLOGY ADMINISTRATION

Robert M. White, Under Secretary for Technology

NATIONAL INSTITUTE OF STANDARDS

AND TECHNOLOGY

John W. Lyons, Director 


\begin{abstract}
As one of the objectives of a program aimed at assessing test methods for in-situ detection of incipient defects in cables due to aging, a laboratory test system was implemented to demonstrate that the partial discharge analysis method can be successfully applied to low-voltage cables. Previous investigations generally involved cables rated $5 \mathrm{kV}$ or higher, while the objective of this program focused on the lower voltages associated with the safety systems of nuclear power plants.

The defect detection system implemented for this project was based on commercially available signal analysis hardware and software packages, customized for the specific purposes of the project. The test specimens included several cables of the type found in nuclear power plants, including artificial defects introduced at various points of the cable.

The results indicate that indeed, partial discharge analysis is capable of detecting incipient defects in low-voltage cables. There are, however, some limitations of technical and non-technical nature that need further exploration before this method can be accepted in the industry.
\end{abstract}




\section{Table of Contents}

1 Summary and Recommendations 1

1.1 Summary of results . . . . . . . . . . . . . . . . . . 1

1.2 Recommendations for future work . . . . . . . . . . . . . 2

1.2.1 Basics on PD phenomena ............... 2

1.2.2 Hardware development . . . . . . . . . . . . . . 3

1.2 .3 Software development. . . . . . . . . . . . . . 3

2 Introduction 5

2.1 The need for cable defect detection ............. 5

2.2 Detection of defects by partial discharge analysis . . . . . . . . 7

3 Review of Defect Detection Methods 8

3.1 General appearance of the material ............. 8

3.2 Mechanical properties of the cable insulation . . . . . . . . 8

3.3 Chemical and physical properties of the insulation . . . . . . . 8

3.4 Electrical properties of the bulk insulation . . . . . . . . . 9

3.5 Detection of point defects . . . . . . . . . . . . 9

4 The Partial Discharge Analysis Method 11

4.1 General ........................... 11

4.2 Point defect detection methods . . . . . . . . . . . 12

5 Partial Discharge Physics $\quad 15$

5.1 Basic physics of partial discharges . . . . . . . . . . . 15

5.2 Measurement of partial discharges (general) . . . . . . . . . 19

5.3 Partial discharge-induced degradation of insulation $\ldots \ldots . . .22$

6 Partial Discharges in Cables 24

6.1 Nature of the measured signals . . . . . . . . . . . . . . . 24

6.2 Cable propagation . . . . . . . . . . . . . . . 28

6.3 Estimation of charge . . . . . . . . . . . . . . 36

6.4 Partial discharge location . . . . . . . . . . . . . . . . 43

6.4 .1 Matched filter . . . . . . . . . . . . . 47

6.4 .2 Multiple sites . . . . . . . . . . . . . . . . 49

6.5 Enhancements in the signal analysis . . . . . . . . . . . . 51

6.5.1 Generalized matched filter . . . . . . . . . . . . 51

6.5 .2 Embedding . . . . . . . . . . . . . . 52

6.5 .3 Cross-correlation . . . . . . . . . . . . . . 52

$\begin{array}{lll}7 & \text { Experimental Apparatus } & 57\end{array}$ 
7.1 Introduction . . . . . . . . . . . . . . . . . 57

7.1.1 Circuit disconnection . . . . . . . . . . . . 57

7.1 .2 Automation . . . . . . . . . . . . . 57

7.1.3 Trend or single measurement. . . . . . . . . . . 58

7.2 Measurement system . . . . . . . . . . . . . . 58

7.2 .1 High voltage system . . . . . . . . . . . 58

7.2.2 PD signal processing system - Initial design . . . . . . . 58

7.2.3 PD signal processing system - Final design . . . . . . . 60

7.3 Data acquisition system . . . . . . . . . . . . . 62

7.4 Data processing system . . . . . . . . . . . . . . 64

7.4 .1 Hardware . . . . . . . . . . . . . . . . . . 64

7.4 .2 System software . . . . . . . . . . . . . . 65

7.4 .2 .1 General ................... 65

7.4 .2 .2 Asystdaq . . . . . . . . . . . 6 66

7.4 .2 .3 Asystred ................... 69

7.4 .2 .4 Asystanl . . . . . . . . . . . 72

8 Experimental Work $\quad 78$

8.1 Artificial versus natural defects . . . . . . . . . . . . 78

8.2 Cable specimens . . . . . . . . . . . . . . . 79

8.2 .1 Simulation of defects . . . . . . . . . . . . 80

8.3 Measurement results ... . . . . . . . . . . . . 83

8.3.1 Initial experiments . . . . . . . . . . . . 83

8.3 .2 Preprocessing . . . . . . . . . . . . . . 87

8.3 .3 Pulse height analysis . . . . . . . . . . . . 88

8.3 .4 Delay analysis . . . . . . . . . . . . . . . . 91

8.3.5 Experimental procedure ................ . . 93

8.3.6 Coaxial cable experiments . . . . . . . . . . . . 94

8.3.7 Multiconductor cable experiments . . . . . . . . . . 95

8.3.8 Two-conductor cable . . . . . . . . . . . . . . 9 95

8.3.9 Effect of test voltage . . . . . . . . . . . . . 96

8.3.9.1 Final blind test ............. 100

9 Discussion of Results $\quad 104$

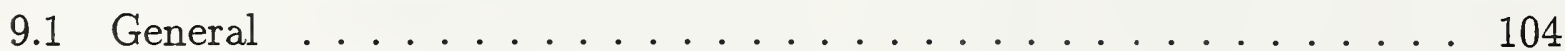

9.2 Technical aspects . . . . . . . . . . . . . . . . . 104

9.2.1 Nature of the cables . . . . . . . . . . . . . . . . 104

9.2.2 Natural vs. artificial defects ................. 104

9.2.3 Practical methods for field tests . . . . . . . . . . . . 105

9.2.4 Operator skill vs. expert system . . . . . . . . . . . . 105

9.2 .5 Test voltage . . . . . . . . . . . . . . . . 106 
9.2 .6 Retrospective .................. 106

10 Possible Further Developments $\quad 107$

10.1 State-of-the-art . . . . . . . . . . . . . . . . 107

10.2 Refinements to defect location techniques . . . . . . . . . . . 108

10.3 Refinements to defect identification techniques . . . . . . . . . . 109

10.3.1 Theoretical modelling and discharge physics . . . . . . . 109

10.3.2 Stochastic analysis . . . . . . . . . . . . . . 111

10.3.3 Artificial enhancement of PDs. . . . . . . . . . . . . 114

11 Conclusions and Recommendations $\quad 115$

11.1 Limitations . . . . . . . . . . . . . . . . . . . 115

11.2 Promising aspects and further work needed . . . . . . . . 116

11.2.1 Technical work . . . . . . . . . . . . . . . 116

11.2.2 Electromagnetic field analysis . . . . . . . . . . 116

11.2 .3 Signal processing . . . . . . . . . . . . . . . 118

11.2.4 Blind spot elimination . . . . . . . . . . . . . . . . 119

11.2.5 Pulsed power supply . . . . . . . . . . . . . . 119

11.2 .6 Cable termination . . . . . . . . . . . . . . . . 119

11.2.7 Defect identification ... . . . . . . . . . . . 119

11.2.8 Acceptance of the method by utilities . . . . . . . . . 120

11.3 Recommendations for action . . . . . . . . . . . . . 120

11.3.1 Basics on partial discharge phenomena . . . . . . . . . . 121

11.3.2 Hardware development . . . . . . . . . . . . . . . . 121

11.3.3 Software development. . . . . . . . . . . . . . . 122

12 Acknowledgements 123

13 References $\quad 124$ 


\section{List of Figures}

5-1. Diagrammic representation of an ac-generated PD process. . . . . 17

5-2. Equivalent circuit of a PD detection system applied to a cable. . . . . 21

6 -1. Elementary circuit diagram for cable . . . . . . . . . . . 28

6-2. Cross-section of coaxial cable . . . . . . . . . . . . . . 29

6-3. Cross-section of two-conductor cable . . . . . . . . . . . . . 30

6-4. Terminated transmission line with PD current source . . . . . . . 32

6-5. Lattice diagram for propagation of $\mathrm{PD}$ pulses . . . . . . . . . . 34

6-6. Lattice diagram and volt-time plot of PD pulses at point within cable 35

6-7. Lattice diagram and volt-time plot of PD pulses at near end . . . . 36

6-8. Relative performance of estimators . . . . . . . . . . . 42

6-9. Delay histogram for two PD sites located $2 \mathrm{~m}$ apart . . . . . . . 50

6-10. Block diagram of generalized cross-correlator . . . . . . . . . . . 54

6-11. Cross-correlation function of a corrupted partial discharge signal. . . 55

6-12. Cross-correlation after pre-filtering . . . . . . . . . 55

7-1. Lowpass filter for high-voltage power supply . . . . . . . . . . . 59

7-2. Circuit diagram and component values for the receiving circuit ... 61

7-3. Sleeve installed over two-conductor cable to minimize PD at the splice 62

7-4. Block diagram of the PD analysis system . . . . . . . . 65

7-5. Flowchart of PD detection . . . . . . . . . . . . 66 66

7-6. Block diagram of Asystdaq . . . . . . . . . . . 67

7-7. Asystdaq main menu . . . . . . . . . . . . . . . 6 67

7-8. Asystdaq parameter menu . . . . . . . . . . . . . . 67

7-9. Asystdaq RECORD CONTENT . . . . . . . . . . . . . 68

7-10. Block diagram of Asystred . . . . . . . . . . . 69

7-11. Asystred main menu . . . . . . . . . . . . . . . 70

7-12. Asystred PARAMETER menu . . . . . . . . . . . . . . 70

7-13. Asystred DATA REDUCTION submenu . . . . . . . . . 71

7-14. Asystanl Block diagram . . . . . . . . . . . . . 72

7-15. Asystanl CALIBRATION menu . . . . . . . . . . . . . 73

7-16. Asystanl PHA menu . . . . . . . . . . . . . . . 74

7-17. PHA Statistics . . . . . . . . . . . . . 75

7-18. Asystanl HISTOGRAM . . . . . . . . . . . . 75

7-19. Aystanl picture of DISCRIMINATION submenu . . . . . . . . 76

7-20. Typical Asystanl PD statistics. . . . . . . . . . . . 76

7-21. Typical Asystanl reduced data in tabular form. . . . . . . . . . . . . 77

8-1. Artificial defects created in two-conductor cable . . . . . . . . . . 81

8-2. Artificial defect created in multiconductor cable . . . . . . . . . 81

8-3. Artificial defects created in coaxial cable . . . . . . . . . . 82 
8-4. Adjacent metal objects near cable ... . . . . . . . . . 83

8-5. Single record of a calibration pulse . . . . . . . . . . . 84

8-6. Response of measuring system to a $0.01 \mathrm{pC}$ PD calibration pulse . . 85

8-7. Histogram of the distribution of $\mathrm{PD}$ pulses . . . . . . . . . . 89

8-8. Typical pulse height distribution. . . . . . . . . . . . . 89

8-9. Histogram of negative partial discharges . . . . . . . . . . 9 90

8-10. Histogram of time delays, converted into feet from the open end . . . 91

8-11. Histogram of delay times between the two major pulses . . . . . . . 9 92

8-12. Histogram of time delays in nuclear-rated coaxial cable . . . . . . . 95

8-13. Histogram of time delays in a multiconductor cable with single defect 96

8-14. Histogram of time delays for $5 \mathrm{kV}$ applied voltage . . . . . . . 97

8-15. Histogram of time delays for $5.2 \mathrm{kV}$ applied voltage . . . . . . . . 98

8-16. Histogram of time delays for $5.5 \mathrm{kV}$ applied voltage . . . . . . . . 98

8-17. Histogram of time delays for $6 \mathrm{kV}$ applied voltage . . . . . . . . 99

8-18. Histogram of time delays for $6.5 \mathrm{kV}$ applied voltage . . . . . . . . 99

8-19. Histogram of time delays for $7 \mathrm{kV}$ applied voltage . . . . . . . 100

8-20. Histogram of time delays at $5 \mathrm{kV}$ and pruned data . . . . . . 101

8-21. Histogram of time delays at $5.2 \mathrm{kV}$ and pruned data . . . . . 101

8-22. Histogram of time delays at $6 \mathrm{kV}$ and pruned data . . . . . . 102

8-23. Emerging defect in the test cable . . . . . . . . . . . 103

11-1. Growth of world-wide literature on partial discharge . . . . . . . 120 


\section{List of Symbols}

Notation

$\alpha \quad$ scale factor in PD estimate 6-39

$\alpha_{i} \quad$ gas ionization coefficient 5-1

$\alpha(\omega)$ attenuation in propagation along the cable $6-24$

$\beta(\omega)$ phase factor of propagation along the cable $6-25$ velocity of propagation in cable $6-22$ permeability of cable conductor $6-14$ reflection coefficient at near end of cable $\ldots \ldots \ldots \ldots \ldots \ldots \ldots \ldots, 6-26$ 
a unknown, nonrandom amplitude scaling factor $\ldots \ldots \ldots \ldots \ldots .6-31$

$\hat{a} \quad$ estimate of the PD pulse amplitude $\ldots \ldots \ldots \ldots \ldots \ldots \ldots \ldots .6 .6 \ldots \ldots$

$\hat{a}_{i} \quad$ estimate of random PD amplitude ..................... 6-40

$\overline{a^{2}} \quad$ mean square value of the PD pulse amplitudes .............. 6-39

$a_{\text {calib }} \quad$ reference level obtained by calibration of measurement system .....6-44

$a_{i} \quad$ random amplitude of $\mathrm{PD}$ impulse $\ldots \ldots \ldots \ldots \ldots \ldots \ldots \ldots, 6,6$

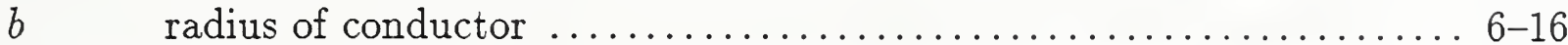

$b \quad$ radius of conductors in parallel wire line $\ldots \ldots \ldots \ldots \ldots \ldots \ldots, 6 \ldots \ldots$

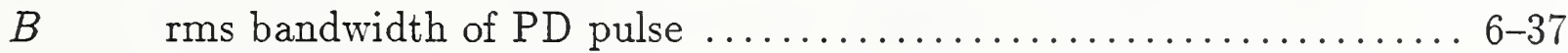

$C \quad$ capacitance per unit length of line $\ldots \ldots \ldots \ldots \ldots \ldots \ldots \ldots .6,12$

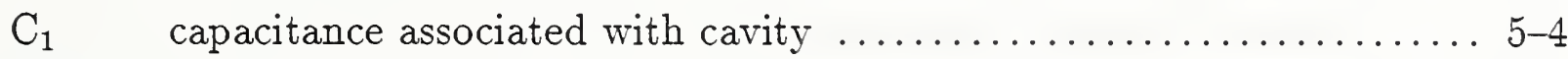

$\mathrm{C}_{2} \quad$ capacitance associated with remaining insulation $\ldots \ldots \ldots \ldots \ldots .5-4$

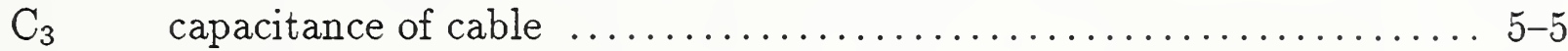

$\mathrm{C}_{\mathrm{k}} \quad$ capacitance of coupling to voltage source $\ldots \ldots \ldots \ldots \ldots \ldots \ldots \ldots$

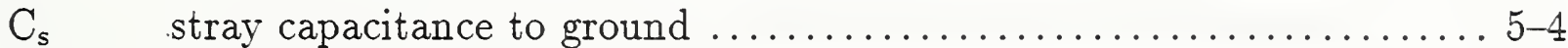

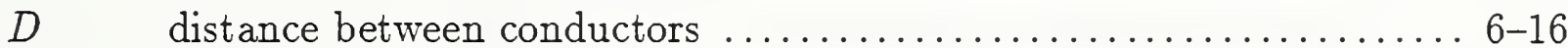

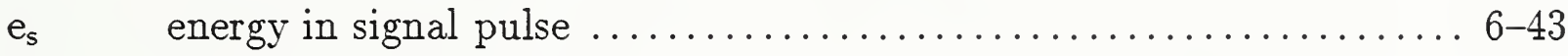

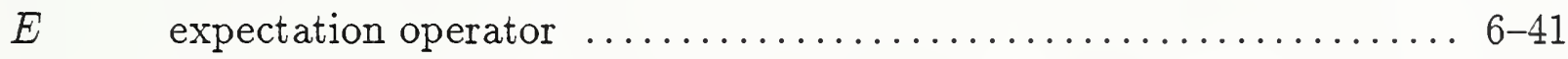

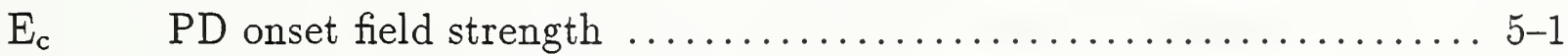

$\mathrm{E} / \mathrm{N} \quad$ electric field-to-gas density ratio $\ldots \ldots \ldots \ldots \ldots \ldots \ldots \ldots \ldots \ldots \ldots \ldots \ldots$

$(\mathrm{E} / \mathrm{N})_{\mathrm{c}}$ critical value, reduced field for $\mathrm{PD}$ onset $\ldots \ldots \ldots \ldots \ldots \ldots \ldots . . \ldots \ldots$

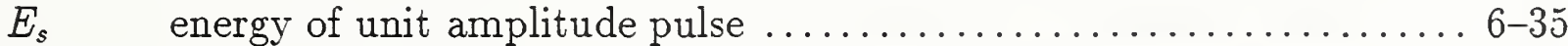

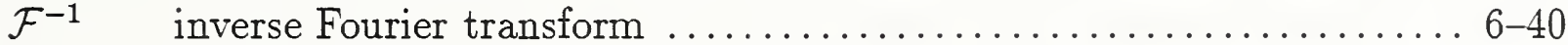

$G \quad$ admittance per unit length of line $\ldots \ldots \ldots \ldots \ldots \ldots \ldots \ldots \ldots, 6 \ldots \ldots$

$h_{c}(t) \quad \mathrm{PD}$ pulse shape determined by cable characteristics $\ldots \ldots \ldots \ldots \ldots 6-6$

$h_{c}(\mathrm{t}, z)$ PD pulse shape at time $t$ and position $z \ldots \ldots \ldots \ldots \ldots \ldots \ldots, 6 \ldots \ldots$

$h_{m}(t) \quad$ direct term in time-reversed calibration $\ldots \ldots \ldots \ldots \ldots \ldots \ldots .6-61$

$H_{c}(\omega)$ propagation characteristic (filter) in loss-less cable ............6-49 49

$H_{e}(\omega)$ bandpass characteristic of instrument ..................... $6-9$

$\mathrm{H}_{\mathrm{G}}(\omega)$ cross-correlation filter function $\ldots \ldots \ldots \ldots \ldots \ldots \ldots \ldots \ldots \ldots \ldots \ldots \ldots \ldots$

$H_{I}(\omega)$ distributed filter of coupling into cable $\ldots \ldots \ldots \ldots \ldots \ldots \ldots \ldots, 6-5$

$H(\omega) \quad$ frequency-domain response of filter $\ldots \ldots \ldots \ldots \ldots \ldots \ldots \ldots, 6$

$\mathrm{H}_{\mathrm{W}}(\omega)$ generalized matched filter function $\ldots \ldots \ldots \ldots \ldots \ldots \ldots \ldots, 60$

$i(t) \quad$ instantaneous current in partial discharge $\ldots \ldots \ldots \ldots \ldots \ldots . . \ldots 5-3$

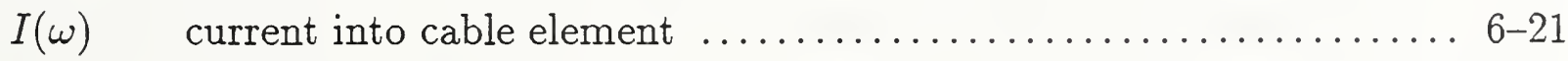


$\overline{I^{2}} \quad$ mean square value of the marks in Poisson process $\ldots \ldots \ldots \ldots \ldots 6-5$

$I_{s}(\omega) \quad$ Fourier transform of a single PD current pulse $\ldots \ldots \ldots \ldots \ldots .6-9$

$k \quad$ Boltzmann's constant ................................6. 6-35

$k_{a} \quad$ attenuation of autocorrelation function $\ldots \ldots \ldots \ldots \ldots \ldots, 6-74$

$k_{c} \quad$ attenuation of correlation function $\ldots \ldots \ldots \ldots \ldots \ldots \ldots .6 .73$

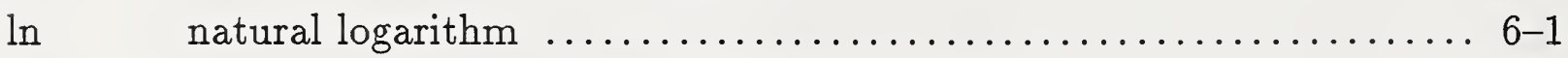

$L \quad$ inductance per unit length of wire $\ldots \ldots \ldots \ldots \ldots \ldots \ldots, 6-15$

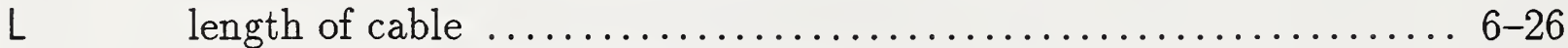

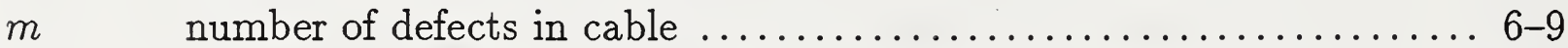

$m(t) \quad$ matched filter in time domain $\ldots \ldots \ldots \ldots \ldots \ldots \ldots \ldots \ldots .6 .61$

$M \quad$ number of Poisson process in cable $\ldots \ldots \ldots \ldots \ldots \ldots \ldots \ldots .6,11$

$M(t) \quad$ matched filter in frequency domain $\ldots \ldots \ldots \ldots \ldots \ldots \ldots .6 .63$

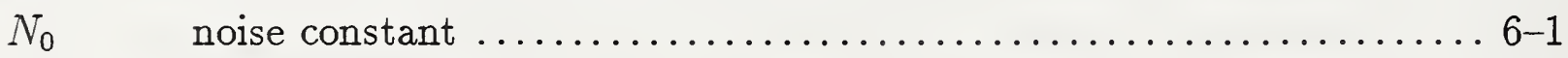

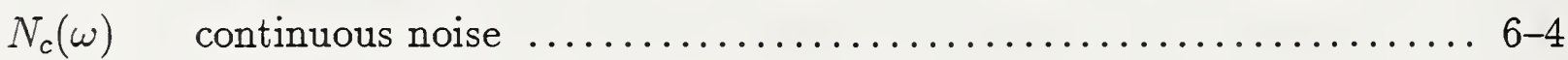

$N_{\Delta t} \quad$ number of PD pulses in time interval $\Delta t \ldots \ldots \ldots \ldots \ldots \ldots, 6-1$

$n_{e} \quad$ number of electrons generated by a partial discharge $\ldots \ldots \ldots \ldots .5-1$

$N(\omega) \quad$ power spectral density of noise $\ldots \ldots \ldots \ldots \ldots \ldots \ldots \ldots, 6,1$

$N_{Q} \quad$ quantization noise $\ldots \ldots \ldots \ldots \ldots \ldots \ldots \ldots \ldots \ldots \ldots \ldots \ldots, 6 \ldots \ldots$

$N_{T} \quad$ number of $\mathrm{PD}$ pulses in time interval $(\mathrm{T}) \ldots \ldots \ldots \ldots \ldots \ldots 6,6$

$p_{\mathrm{d}}^{\prime} \quad$ probability per unit time for release of an electron $\ldots \ldots \ldots \ldots \ldots .5-2$

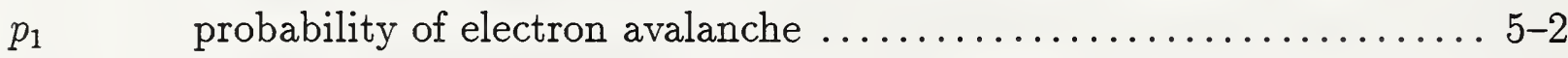

$q \quad$ charge transfer in a single $\mathrm{PD}$ pulse $\ldots \ldots \ldots \ldots \ldots \ldots \ldots \ldots \ldots, 3$

$Q_{\min } \quad$ minimum measurable charge transfer in PD pulse $\ldots \ldots \ldots \ldots .6-36$

$r_{1} \quad$ radius of core conductor in coaxial cable $\ldots \ldots \ldots \ldots \ldots \ldots .6-12$

$r_{2} \quad$ radius of shield of coaxial cable $\ldots \ldots \ldots \ldots \ldots \ldots \ldots \ldots, 6,12$

$R \quad$ resistance per unit length of line $\ldots \ldots \ldots \ldots \ldots \ldots \ldots \ldots, 6-14$

$R_{m} \quad$ resistance of PD measurement element .................. 5-6

$s_{s}(t) \quad$ unit amplitude of single PD pulse $\ldots \ldots \ldots \ldots \ldots \ldots \ldots \ldots \ldots .6 .6 \ldots \ldots$

$s(t) \quad$ noiseless received PD signal in time domain $\ldots \ldots \ldots \ldots \ldots \ldots .66$

$S_{\text {direct }}^{*}(\omega)$ the directly arriving component of the time-reversed PD pulse ... 6-62

$S(\omega) \quad$ power spectral density of Poisson process $\ldots \ldots \ldots \ldots \ldots \ldots \ldots 6-7$

$\mathrm{SNR}_{\text {out }} \quad$ subjective criterion of measurement quality $\ldots \ldots \ldots \ldots \ldots \ldots 6-35$

$S_{s}(\omega) \quad$ noiseless received PD signal in frequency domain $\ldots \ldots \ldots \ldots \ldots 6-31$

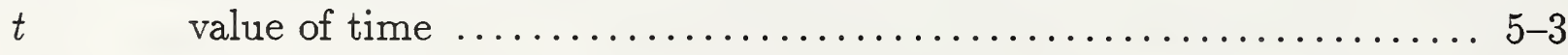

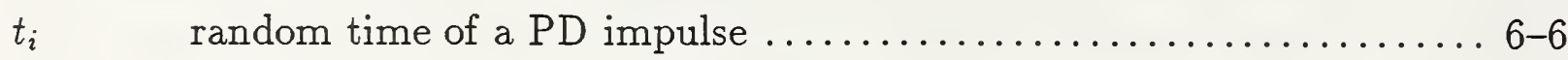




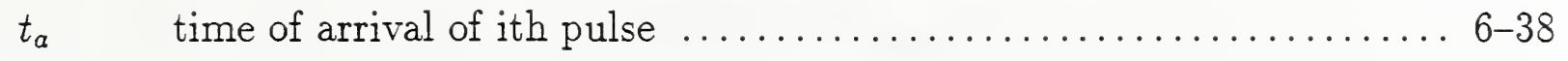

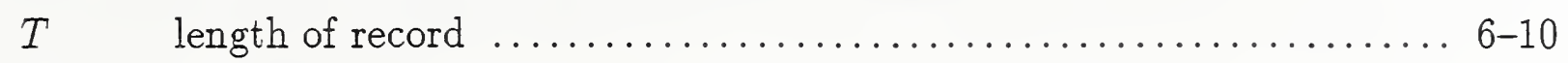

$T_{e q} \quad$ equivalent noise temperature of measurement system $\ldots \ldots \ldots \ldots 6-35$

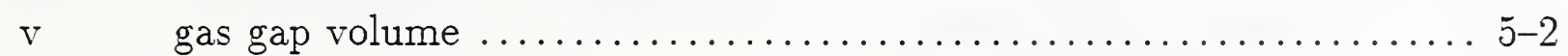

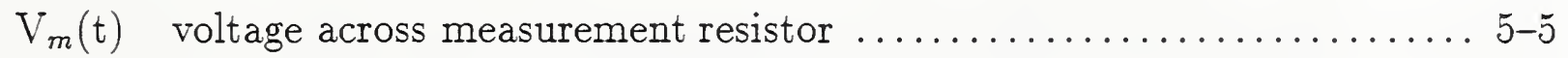

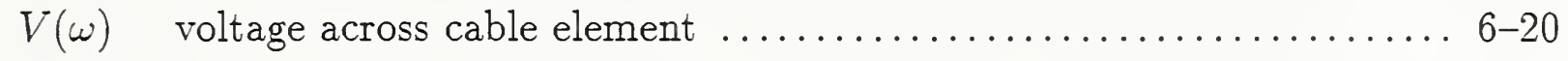

$x_{i} \quad$ location (distance) along the cable length $\ldots \ldots \ldots \ldots \ldots \ldots \ldots 6-26$

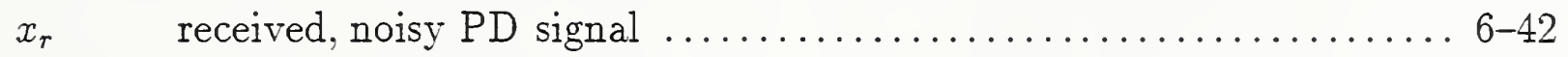

$\vec{x} \quad$ distance along path of electron avalanche $\ldots \ldots \ldots \ldots \ldots \ldots \ldots . \ldots .2$

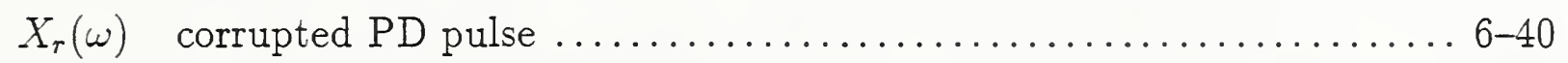

$y \quad$ value of time autocorrelation function $\ldots \ldots \ldots \ldots \ldots \ldots \ldots, 42$

$z_{i} \quad$ specific location of a partial discharge $\ldots \ldots \ldots \ldots \ldots \ldots .6 .6 .6$

$Z_{0} \quad$ characteristic impedance of cable ...................... 6-23

$Z_{f} \quad$ terminating impedance at far end of cable $\ldots \ldots \ldots \ldots \ldots \ldots \ldots .6 .29$

$Z_{n} \quad$ terminating impedance at near end of cable $\ldots \ldots \ldots \ldots \ldots \ldots .6-28$

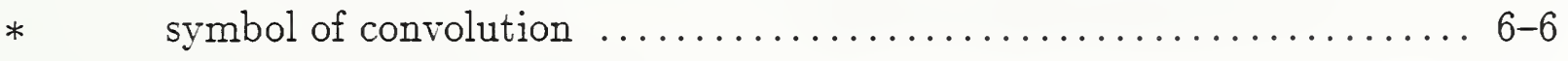

* symbol of complex conjugate $\ldots \ldots \ldots \ldots \ldots \ldots \ldots \ldots \ldots \ldots .6 \ldots \ldots$

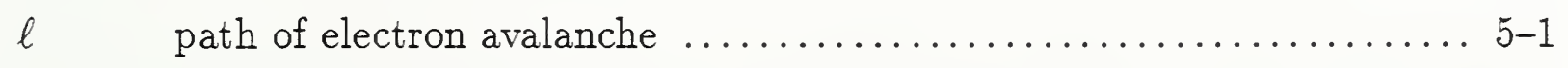




\section{List of Acronyms}

$\begin{array}{ll}\text { AWG } & \text { American Wire Gage } \\ \text { CPU } & \text { Central Processing Unit } \\ \text { DTDR } & \text { Differential Time-Domain Reflectometry } \\ \text { GPIB } & \text { General Purpose Interface Bus } \\ \text { IEEE } & \text { Institute of Electronics and Electrical Engineers } \\ \text { IR } & \text { Insulation Resistance } \\ \text { LOCA } & \text { Loss of Cooling Accident } \\ \text { LMMSE } & \text { Linear Minimum Mean Square Error } \\ \text { NIST } & \text { National Institute of Standards and Technology } \\ \text { PC } & \text { Personal Computer } \\ \text { PHA } & \text { Pulse Height Analysis } \\ \text { PIG } & \text { Pre-Ionized Gas } \\ \text { RF } & \text { Radio Frequency } \\ \text { SNR } & \text { Signal-to-Noise Ratio } \\ \text { TDR } & \text { Time-Domain Reflectometry } \\ \text { TDS } & \text { Time-Domain Spectroscopy }\end{array}$




\section{Summary and Recommendations}

\subsection{Summary of results}

As one of the objectives of a program aimed at assessing existing test methods for in-situ detection of incipient defects in cables due to aging, a laboratory test system was implemented to demonstrate that the partial discharge (PD) analysis method can be successfully applied to low-voltage cables. Previous investigations generally involved cables rated $5 \mathrm{kV}$ or higher, while the objective of the program focused on the lower voltages associated with the safety systems of nuclear power plants.

The system implemented for this demonstration was based on commercially available signal analysis hardware and software packages, customized for the specific purposes of the project. The test specimens included several cables of the type found in nuclear power plants, with artificial defects introduced at various points of the cable. The final demonstration of the system included a cable where several defects were inflicted at locations kept undisclosed to the test operator. Using a combination of the standard analysis built into the system and experience-based detailed analysis of selected portions of the data base, the operator was able to identify the existence and location of all these undisclosed defects.

These results indicate that, indeed, PD analysis is capable of detecting incipient defects in low-voltage cables. There are, however, some limitations of technical and non-technical nature that need further exploration before this method can be accepted in the industry:

- The inception voltage for a cable containing a defect does not appear to be lower than the inception voltage for a cable that is free of defects. Thus, a relatively high voltage is necessary, which may become objectionable to power plant operators. Thus, an "education" program will be necessary to gain acceptance of the method by industry.

- This relatively high voltage is most likely unacceptable for the equipment connected at the far end of the cable. Thus, a requirement for applying the partial discharge test will be that equipment be disconnected at the far end of the cable.

- In its present configuration, connections of the test leads to the cable to be tested have been the sites of substantial discharges that tend to drown out the discharges originating from defect sites. While in principle the signal processing should be able to discriminate these, they place an added burden on the system.

- Unshielded, single conductor cables (unless part of a bundle) may not be amenable to the detection of partial discharges resulting from an incipient 
defect. Similarly, in a two-conductor cable a defect limited to one side of one conductor, oriented away from the other conductor, has a low probability of detection.

- The ultimate goal of having the personnel working in nuclear power plants, who are skilled but not highly specialized in partial discharge detection techniques, perform the in-situ tests will require a substantial amount of signal processing and "expert system" development - an activity that was not included in the scope of the project.

\subsection{Recommendations for future work}

These recommendations are offered from the technical point of view, independently from considerations of budget priorities and scope of sponsoring organizations. Three areas of investigations can be considered: basics of partial discharge phenomena, hardware development, and software development.

\subsubsection{Basics on PD phenomena}

Little information is available on the nature of partial discharge occurring at the site of an incipient defect. Thus, cables removed from the field and presumed to contain incipient defects should be subjected to laboratory tests to investigate the nature of partial discharge in such cables, as opposed to those with artificial defects.

Once the PD pulse has been launched in the cable, the propagation characteristics of that cable will influence the waveform of the pulse arriving at the instrument. Hence, better understanding of propagation characteristics is needed to take advantage of methods that include the waveform patterns in the analysis of the data.

Different cable geometries are stressed differently when high voltage is applied. Fundamental to the success of the method is the understanding of the relationship between the applied voltage and the electric field applied across the dielectric. Experimental and numerical modeling would greatly aid in the understanding of this relationship and the level of applied electric field necessary to cause partial discharge.

The use of ionizing gases has been established as one means of reducing the voltage necessary to reach the PD inception voltage. Technology is readily available for pumping gas down the conductors of insulated wires. This is one avenue that could prove fruitful as an enhancement to the existing method.

The method depends upon the statistical nature of partial discharge. Very little information is available on the statistical nature of the partial discharges due to defects as well as the partial discharges due to innocuous anomalies in insulation. 
Statistical measures could be used to discriminate between innocuous background partial discharges and those due to defects.

\subsubsection{Hardware development}

One of the limitations of the PD analysis method as implemented during this project is the volume of acquired data that must subsequently be discarded by the softwaredriven analysis. Discrimination against these data by hardware filtering before analysis should be investigated to reduce the volume of data processed by the software.

The data-acquisition hardware (digitizing oscilloscope) used in the experimental work of this project is, by today's fast-moving standards, antiquated. Similar digitizing oscilloscopes are now available that can capture the same amount of data in 30 seconds that previously took 20 minutes. Using up-to-date hardware would dramatically reduce the time required for analysis.

Practical methods are needed for attaching the test equipment to the cable under test in such a manner that no overpowering partial discharge will occur at the point of connection.

One of the primary goals of the instrumentation system should be to reduce the duration of exposure of the cable to high voltage during the test. Using a power supply that can be energized under computer control would permit the computer to apply voltage only during the time when the data acquisition system is acquiring data. The high roltage would then be turned off during data transfer and archiring procedures.

Special-purpose hardware is now arailable that can dramatically increase the computational abilities of standard PC computers. Addition of this hardware could reduce the analysis time from several hours to a fer minutes. The general procedure in this project was to collect data during the day and analyze data during the evening. Based on the results, further experiments were planned for the next day. With accelerated processing more experimentation would be possible.

Testing with a $60 \mathrm{~Hz}$ transformer is cumbersome because of the size of the test equipment. Alternative methods should be inrestigated that eliminate bulky equipment.

\subsubsection{Software development}

Three tasks have been identified as potential or necessary enhancements to the PDA method as demonstrated during this project: 
1. Develop a generalized matched filter which would improve the PD pulse recognition for analysis after the attenuation along the cable.

2. Develop a cross-correlation method that can be applied when both ends of the cable are available. With the likely necessity of disconnecting any equipment at the far end of the cable, this is a realistic opportunity.

3. Last but not least, the success and eventual acceptance of the method demand that the equipment be operated by maintenance personnel available to the utilities, not by highly specialized experts on PD technology. Therefore, the expertise of the specialists needs to be converted into a data base accessible to an expert system incorporated with the instrument that will assist the maintenance personnel in performing meaningful tests. 


\section{Introduction}

\subsection{The need for cable defect detection}

Among the many components being considered for assessment of aging and residual life of nuclear power plants, cables merit consideration because of their unique role in plant safety and support systems. Stresses imposed on the cables include mechanical stresses at the time of construction and installation, followed by many years of exposure to the conditions of the operational environment. Aging takes place under the combined effects of humidity, heat, oxidation, and radiation associated with each cable system. And yet, in accident situations, the aged cables are expected to withstand the mechanical and environmental stresses associated with such an event and perform their intended function. This situation has not escaped the attention of designers, plant operators and regulators. The detection of incipient defects has become a recognized subject of study. This report presents the results of a project aimed at assessing applicability of known insulation test methods for in-situ detection of incipient defects.

A review of existing practices and of the literature on insulation evaluation tests shows that existing power plant facilities depend mainly on the use of cables that have been qualified in accordance with appropriate national codes and standards prior to installation and operation. There are ongoing programs, however, for assessing the aging of installed cables, but no simple method has been found for performing nondestructive in-situ tests. There are many recognized tests for assessing the condition of cable insulation but most of them are destructive and typically performed in the laboratory on specimens that include new cables, artificially aged cables, and naturally aged cables. The literature shows that tests on artificially aged cables, where the aging is accelerated by one or more environmental overstresses, are often subject to debate on the validity, conservatism, or ambiguity of the acceleration process (Jaworska et al., 1983).*

The detection of incipient cable defects, especially for the safety-related cables of a nuclear power plant, is an important aspect of assessing the operational integrity of a power plant. Existing cable systems in nuclear power plants have been installed on the basis of qualification tests with the expectation that aging of the insulation under the prevailing environmental conditions will not result in defects within the design lifetime of the cable (Petty, 1988). As the existing plants advance in age and ultimately reach their rated life, renewal of the plant license will require a demonstration that there is adequate residual life in the cable insulation (Ahmed et al., 1985; Vora, 1986; Jacobs,

*Bibliographic citations are listed alphabetically in Section 13. 
1987; Bustard, 1987). The detection of incipient defects would indicate inadequate residual life. Conversely, the absence of incipient defects, if it could be established with absolute certainty, would indicate adequate residual life. This oversimplified dichotomy, however, is not what prevails in the actual situation.

Realistically, after a nearly century-long quest by many researchers in the industry for improving methods for the evaluation of the condition of an insulation system (Brancato, 1978; Bartnikas, 1987), some approaches have gained acceptance. However, based on a correlation between the results of laboratory tests and field experience, but no absolute certainty can be offered. As promising new insulation materials are introduced and as experience sorts out the successful from the less successful materials, the goal is a moving target. Furthermore, there is a wide variety of potential defects as well as a wide variety of accepted test methods; hence, expecting that a single method will detect all possible defects would be totally unrealistic. What can be expected is that, through the cumulative knowledge gained from field experience and from an array of laboratory tests, enough understanding of the insulation condition can be gained to make a determination of residual life.

This report describes in detail one promising method for the detection of a family of defects. According to the scope of the work reported here, the objective was to investigate the possibility of applying a known method, based on the processing of partial discharge (PD) signals, to the detection of incipient defects - not to invent a new method. To place this one method in perspective, the following two sections give a brief overview of the types of possible defects and a review of basic test methods. Later sections present a detailed discussion of the physics of partial discharge, leading to a discussion of the nature and propagation of partial discharge signals in cables and their detection and processing through an experimental apparatus assembled during the course of this project.

A very important concept for in-situ insulation evaluation through nondestructive test methods is that trends in test results can yield much more information than absolute numerical values obtained from single tests. Therefore, any program aimed at insulation evaluation is likely to require establishing and updating a data base on test results. Thus, in addition to the technical challenge of the test method itself, a significant part of the total challenge will be the logistics of managing the data base. A further challenge is the credibility of conclusions based on small changes observed over a long period of time by tests methods that have yet to be generally accepted. One aspect of acceptability criteria is that test methods should be simple enough to be performed by the personnel normally available in nuclear power plants, with minimum subjective interpretation of the results. 


\subsection{Detection of defects by partial discharge analysis}

Partial discharges occur in insulation systems when the local field strength exceeds the withstand capability of the gaseous medium surrounding the site. In a cable system, such discharges produce a pulse that propagates on both sides of the site of the discharge, and can be monitored at the end of the cable. For the purpose of this discussion, the cable of interest can be a power, control, or signal cable. One end, ('near end'), typically outside the containment vessel, is presumed to be readily accessible to the personnel performing the test, while the other end ('far end'), typically within the containment vessel, is less accessible. If the cable is not terminated in its characteristic impedance at the far end, a reflection occurs when the PD pulse arrives at this far end. The pulse travels back toward the near end. By recording the amplitude and time of arrival of the two pulses - the one coming directly and the one arriving after reflection at the far end - it is possible to obtain information leading to identification and location of the site of the partial discharge.

This method was first described for medium-voltage cables (Weeks \& Steiner, 1982). As it appeared promising for the lower voltage cables of nuclear power plant safety systems, the project team approached Weeks and Steiner to propose a joint evaluation of the applicability of their method to the lower voltage cables. A PD analysis system emulating the one developed by Weeks and Steiner at Purdue University was assembled at the National Institute of Standards and Technology (NIST) for the purpose of assessing the capability of the method in the context of low-voltage cables. This report describes the results obtained from the NIST system. To place this method in perspective, a brief review is made in the next section of the methods that have been described in the literature (Martzloff \& Perrey, 1991) for the detection of defects in insulation systems, cables in particular. 


\section{Review of Defect Detection Methods}

There are several approaches for assessing the condition of an insulation system, involving different characteristics of the insulating material. Some of these are described in detail by recognized industry standards (Garner \& Winslow, 1988; Gradin, 1988) while others are part of the empirical knowledge of specialists, or are still under development (Mopsik et al., 1988). For the purpose of placing these in context, these approaches may be classified in five types, as summarized below.

\subsection{General appearance of the material}

A visual inspection of an insulation system may not appear scientific, but experienced personnel can obtain valuable information from such an inspection. In the case of cables, however, a visual inspection is impractical; furthermore, the constraints of a determination for the purpose of license renewal may limit the acceptability of such a source of information. We cite it here, however, as a reminder that experience of insulation specialists remains a strong factor in the pursuit of realistic and successful test methods (Mathes, 1987; EPRI Workshop, 1988).

\subsection{Mechanical properties of the cable insulation}

During installation, during normal operation in a plant, and during abnormal conditions, in particular during a loss of coolant accident (LOCA), the cable insulation is subjected to mechanical stress. Adequacy of a cable to withstand these stresses is established by subjecting new or aged cable specimens to a battery of tests that include bending the cable around a mandrel and performing elongation/rupture tests on specimens cut from cable samples (Jeppeson, 1982). Thus, these tests are essentially destructive. A recent proposal has been made to turn an old empirical test into a well-controlled, non-destructive test: measuring the stress/strain characteristic of the cable insulation, in the field, with a portable anvil-indenter system (Toman \& Gardner, 1986; Gardner \& Shook, 1988). Initial results of this approach have been encouraging.

\subsection{Chemical and physical properties of the insulation}

Degradation of the insulation is often associated with changes of the chemical composition, molecular structure, or physical state of the material (Mopsik, 1991). 
Examples of changes that can be detected by appropriate methods include oxidation (Clough \& Gillen, 1982; Stonkus, 1988; Margulis et al., 1983), splitting off of $\mathrm{HCl}$ from polyvinyl chloride (Wilson, 1974; Salovey; 1973), and cross linking or chain scission (Mandelkern, 1972; Geymer, 1973). The changes can be induced or accelerated by exposure to ultraviolet or ionizing radiation (Hedwig, 1972).

\subsection{Electrical properties of the bulk insulation}

Several types of electrical characteristics can serve in assessing the condition of the insulation: dielectric properties of the bulk material, and the magnitude of anomalies in the uniformity of the insulation. Dielectric properties of the bulk material do not directly signal the presence of an incipient defect, but do provide an indication that the material properties are changing, with a possibility (to be established by suitable correlation) that the integrity of the insulation may be in jeopardy. A promising example of such an approach is the time-domain spectroscopy (IDS) method (Mopsik, 1984), which has been applied during this project and is reported in NISTIR-4486 (Mopsik, 1992). Measurement of dc insulation resistance, dielectric losses, and leakage current have also been applied and remain to this day among the principal evaluation methods (Lee, 1977; Kwaaitaal \& van den Eijden, 1987; Phillips, 1988; Stone et al., 1988). However, when applied in-situ to a cable system (in contrast to a sacrificial cable specimen) these methods cannot easily separate intrinsic insulation condition from surface conditions.

\subsection{Detection of point defects}

As opposed to bulk changes in dielectric properties, cable insulation may also be compromised by point defects resulting from a variety of causes such as small defects in the manufacturing process that grow later on, partial damage during installation, or cracks dereloping as a result of aging. If large enough, these defects produce a localized change in the cable impedance that can be detected by time-domain reflectometry (TDR) (Meininger \& Dinsel, 1985; Dinsel et al., 1986; St. Onge, 1988). However, a small defect, such as a single crack that could become a fault under LOC.A conditions, cannot be detected under normal entironment conditions (Anderson et al., 1985; 1986). Another approach has been used for many years, involving the detection of partial discharge under normal utilization voltage or under some degree of overvoltage stress (Bever et al., 1982: Bartnikas, 1990). Progress during the last decade in signal processing technology has opened new opportunities for the detection and interpretation of partial discharges (Weeks \& Steiner, 1982; 1985; Knapp et al., 1990; Mashikian et al., 1990). These promising approaches, especially for mediumvoltage ranges $(5-35 \mathrm{kV})$ were identified in the early stages of this project, leading to the implementation of a test system at NIST. 
Unresolved questions at the outset of the implementation of this test system included:

- possible limitations resulting from the lower voltages, compared to the original Weeks-Steiner demonstration

- effect of multiple PD sites

- possibility of transferring the technique from the laboratory to the environment of a nuclear power plant (operational limitations, electromagnetic compatibility, personnel availability)

This report describes the successful implementation and demonstration of a test system for $\mathrm{PD}$ analysis (PDA) in a laboratory environment. In order to understand the implications of the approach, a discussion is first provided in the next two sections of the physics of partial discharge in general, and of its occurrence in cables in particular. 


\section{Partial Discharge Analysis Method}

\subsection{General}

To state that any single test will adequately characterize an insulation system is misleading since a myriad of material properties are needed to properly characterize any insulation system. The purpose of this section is to bring into context the partial discharge analysis (PDA) method by describing the relationship to and differences between the PDA method and other insulation measurement methods. Several definitions are required to develop a general context for insulation testing so that the methods might be compared.

There are a variety of measurement methods used to characterize insulation systems and each approach has as an objective the measurement of the insulation's degradation. Degradation can be broadly categorized into two groups according to the material properties of interest. For the purposes of this report, degradation is classified either as bulk deterioration or as a point defect, which are defined as:

- Bulk deterioration is an undesirable change in the bulk material properties of the insulation system. An example of bulk deterioration is a change in the insulation resistance of the material. Bulk deterioration does not necessarily indicate the presence of incipient faults in the insulation system, only the increased likelihood of their presence.

- Point defects are flaws that exist at localized positions within the insulation system. Examples of point defects are cracks or thinning in the insulation of a cable. A point defect is an incipient fault in the insulation that could lead to failure of the insulation system given the proper circumstances.

Insulation tests provide different forms of information about the insulation system under test. This information either characterizes the insulation system as a whole or characterizes specific points within the insulation system. This measurement information can be categorized into two groups, global information or local information, which are defined as:

- Global information is information that characterizes the properties of the insulation system as a whole. An example of global information is the result from an insulation resistance test which is a measure of the insulation resistance (IR) of the entire system. Another example is when a cable passes a high-roltage test ("hi-pot"), the cable is said to have a certain dielectric strength.

- Local information is information that characterizes the material properties of the insulation system at specific locations. An example of local information is the result from a time-domain reflectometry (TDR) test which characterizes 
the impedance discontinuities at specific points along the length of a cable. Another example is when a cable fails a hi-pot test, the cable lacked the required dielectric strength at the point where it failed.

The administration of insulation tests can be categorized into two groups according to the point of application, terminal testing or spot testing, which are defined as:

- A terminal test is defined as a test used to measure the entire insulation system from a single terminal or access point. Both TDR and IR tests are terminal tests since they are performed at a single position along the insulation system.

- A spot test is one which performs the test at a specific point in the insulation system. An example of a spot test is the thumbnail test that measures the brittleness of the insulation at a single point.

\subsection{Point defect detection methods}

The PDA method can be characterized using several of the definitions given above. The PDA method is a terminal test since it tests the entire insulation system from a single position. The PDA method of testing measures point defects in the insulation system since it characterizes weaknesses in the dielectric strength of the insulation at specific points. The PDA method provides local information about the insulation system in the case where it detects flaws but also provides global information about the insulation system in the sense that it characterizes the dielectric strength of the entire system.

Three other insulation tests, the hi-pot test, the TDR test and pre-ionized gas (PIG) test, all have the same attributes as the PDA method. As will be seen, the PDA method is nothing more than an extension of these tests. In fact, the PDA method, because of its implementation, is capable of providing the same information as the other tests along with additional information that the other methods are incapable of providing.

The hi-pot test applies a high voltage, ac or dc, to the cable system and determines the integrity of the cable on the basis of whether or not the insulation fails. The PIG test is essentially a sophisticated ac hi-pot test since the PIG method also applies a high voltage to the cable system and determines the integrity of the cable on the basis of whether the insulation fails. The main difference between the hi-pot test and the PIG test is that in the PIG test a rare gas is introduced to lower the ionization potential of the gas surrounding the cable. The result is that the PIG test can use lower voltages to achieve breakdown. There appears to be some evidence that the gas surrounding the cable may ionize more completely when the PIG test is used. Also, when the cable fails during this test, there is very little carbonization of the 
fault site. The advantage that these tests have is that there is a strong correlation between dielectric strength of the insulation and the presence of point defects.

While the PDA method is somewhat related to the hi-pot and PIG tests, since all three use partial discharge, there are several distinctions. The hi-pot and PIG test do not directly measure the partial discharge but use it as a means to fail the sample. In contrast, the PDA method directly measures the partial discharge and uses its properties to perform an analysis on the cable. One of the main differences between the PDA method and the hi-pot test or PIG test is that more of the available information is used to determine the state of the insulation. The chief advantages of the PDA method compared to the hi-pot and PIG tests are:

- The ionization is minimized which in turn minimizes any damage to the insulation system.

- The cable under test is not stressed to breakdown, allowing continued function of the cable tested until replacement can be effected.

- Adjacent cables are not in peril of being damaged by failure of the specimen under test.

- Multiple point defects can be identified.

- Appropriate implementations of the PDA method can also perform a hi-pot or PIG tests, if necessary.

- Rather than being used an alternative to the hi-pot or PIG test, the PDA method could replace these tests.

The PDA method can also be considered to be a variation on TDR in the sense that time delays are determined from pulses reflected in the cable under test. There are several differences between the two methods. In the PDA method, the pulses used as the probing signal are generated by the point defect rather than by an external source; the signal source is a passive source. The only reflection of interest in the PDA method is the reflection of the probing signal from the open circuit end of the cable. In contrast, TDR uses an external source that generates the pulses; the signal source is an active source. All reflections in the cable under test are important for the TDR method and are interpreted in terms of discontinuities caused by flaws.

Anderson showed that TDR is not capable of detecting cracks or channels in practical insulation systems (Anderson et al., 1982; 1986) while the PDA tests main objective is to detect these. One step in the PDA method is calibration in which the system operates in a TDR mode to determine the propagation characteristics of the cable. Since the system is capable of operating in a TDR mode, it can make any measurements that a TDR can. The chief advantages of the PDA method compared to the TDR method are: 
- The PDA method can detect small cracks and fissures in the cable insulation which are invisible to TDR.

- The PDA method is capable of incorporating all aspects of the TDR method in its implementation, plus what the TDR cannot accomplish.

As will be discussed in subsequent sections, PD signals contain a wealth of information about the defect. Not only do these signals provide an indication of where the defect is located, but also there is hidden information contained in amplitudes and occurrence times of the partial discharges. It may be possible to extract this information and use it to enhance the resolution of the analysis. In any type of insulation measurement, experience must be built so that the measurements can be properly interpreted. Take for instance the insulation resistance. (IR) test: this test is widely accepted to determine the integrity of an insulation system and is classified as a terminal test and as a global test. Consider, for example, that the IR measurement is made on an insulation system. Using this measurement, it is desired to determine whether the insulation is failing. If a data base is available that documents the failures of similar insulation systems as a function of the IR measurement, then one could draw a conclusion from this reading.

Another more sophisticated example of data base use can be found in the application of TDR to cable systems. The interpretation of TDR signals can be extremely difficult. TDR is relatively insensitive to all but the most severe point defects. Typically a short or open circuit must be present to unequivocally determine that the cable has failed. The use of differential TDR (DTDR) provides a moderate improvement in the detection of flaws. The use of DTDR requires the accumulation of an extensive data base for the particular cables under examination. The cables of interest usually have poor propagation characteristics resulting in TDR signals that have significant clutter masking the flaws. Recording the TDR signature of the cable before it fails allows the operator to subtract the clutter from subsequent measurements making the newly developed flaws more apparent. On the surface, this technique seems to provide a substantial improvement in performance, however, environmental factors cause significant drift in the mechanisms causing the clutter. As a consequence, it is only possible to remove a portion of the clutter which means that DTDR can still only detect relatively large flaws. The question remains whether minor impedance discontinuities revealed by this method indicate a failing insulation system. Once again, it is required to have a secondary data base documenting the failure of similar cable systems as a function of detected impedance discontinuities.

The general idea illustrated by the preceding examples is that extensive data bases are required to make decisions based upon a measurement of the insulation system. Regardless of the sensibility of the measurement method employed, it cannot provide the user with a definitive answer about the integrity of the insulation system unless it is supported by an extensive data base gathered through experience. 


\section{$5 \quad$ Partial Discharge Physics}

\subsection{Basic physics of partial discharges}

A partial discharge (PD), as the name implies, is an electrical breakdown phenomenon that is confined to localized portions of the insulating space between two conductors at different potentials. The localization of the discharge is generally the consequence of an electric field enhancement confined to a region that is relatively small compared with the dimension of the gap between the conductors. The field enhancement can be associated with abrupt changes in the nature of the insulating medium such as might be caused by voids in solids or gas-filled spaces at the interfaces between dielectricconductor or dielectric-dielectric surfaces.

Partial discharge is basically a phenomenon that occurs in gases, i.e., the presence of the gas phase is required for its occurrence. Although recent advances have shown that partial discharges are also found to occur in liquids (Kelley et al., 1989; Van Brunt et al., 1992) the formation of an ionized channel associated with the electrical discharge requires that the liquid first be vaporized, i.e., that gas "bubbles" be formed. Partial discharges that are purported to occur "in" solid insulation actually develop within voids or cracks that contain gas, or along the surfaces at gas-solid interfaces. Partialdischarge activity in cables insulated with polymer materials is often associated with the onset of "trees" (Dissado \& Hill 1990; Huzumi et al., 1988) formed during the process of aging. These trees are composed of either gas-containing micro channels or material of low density that can be readily vaporized such as water (Bulinski et al., 1988; Dissado, 1982).

A necessary condition for $\mathrm{PD}$ formation in a volume containing an electronegative gas is that a portion of the volume be at an electric field-to-gas density ratio $(E / N)$ that is greater than or equal to the critical value $(E / N)_{c}$ at which the gas ionization coefficient $\alpha_{i}$ equals the electron-attachment coefficient $\eta_{a}$ i.e., $\alpha_{i}=\eta_{a}$ (Van Brunt, 1987). If this condition is satisfied, then a $P D$ can form when an initiatory electron is injected into the volume where $(E / N)>(E / N)_{c}$. The initiatory electron could, for example, result from field emission if the electric field at the surface is sufficiently high, or from ionization of a gas molecule by external radiation such as associated with naturally occurring cosmic rays.

In order that the PD be detectable, the amount of charge (approximately equal to the number of electrons produced) in the PD must exceed a level $\mathrm{q}_{c}$ determined by the sensitivity of the detection circuit used. If, for example, the detector is sensitive to discharges down to the $0.05 \mathrm{pC}$ level, then the number of electrons generated by the $\mathrm{PD}, n_{e}$, must be greater than $3 \times 10^{5}$. For $n_{e} \leq 10^{8}$, the $\mathrm{PD}$ can be thought of 
as an electron avalanche for which $n_{e}$ is given by (Legler, 1961)

$$
n_{e}=\exp \left(\int_{\ell}\left[\alpha_{i}(\vec{x})-\eta_{a}(\vec{x})\right] d \ell^{\prime}(\vec{x})\right),
$$

where $\ell$ represents the path followed by the electron avalanche in the gas gap in which $\mathrm{E} / \mathrm{N} \geq(\mathrm{E} / \mathrm{N})_{c}$ and $d \ell^{\prime}(\vec{x})$ is an element of path length. Equation 5-1 corresponds to an "average" avalanche initiated by a single electron.

The probability per unit time that an observable PD having more than $n_{e}^{\prime}$ electrons will be formed at a given location $\vec{x}_{o}$ in a gas filled gap of volume $\mathrm{v}$, where $(E / N)>$ $(\mathrm{E} / \mathrm{N})_{c}$, can be expressed by the integral

$$
p\left(n_{e}>n_{e}^{\prime}\right)=\int_{\mathrm{v}} p_{d}^{\prime}\left(\vec{x}_{o}\right) \int_{n_{e^{\prime}}}^{\infty} p_{1}\left(n_{e} \mid \vec{x}_{o}\right) d n_{e} d^{3} x_{o}
$$

where $p_{d}^{\prime}\left(\vec{x}_{o}\right)$ is the probability per unit time for release of an electron at $\vec{x}_{o}$ and $p_{1}\left(n_{e} \mid \vec{x}_{o}\right)$ is the probability that the released electron results in an avalanche (or $\mathrm{PD}$ ) of $n_{e}$ electrons. The form for the function $p_{d}^{\prime}$ is determined by the statistical nature of the electron release process and $p_{1}\left(n_{e} \mid \vec{x}_{o}\right)$ is controlled by the stochastic behavior of avalanche (or PD) growth which can be predicted under some well-defined conditions (Legler, 1961; Van Brunt, 1986).

The PD phenomenon of interest here is a pulsating phenomenon in the sense that it is manifested by the occurrence of discrete current pulses. The pulsating behavior results from the fact that partial discharges are inherently self-choking. As the discharge current pulse develops, it leaves behind surface charges or localized ion space charges that reduce the electric field to the point where the condition $\mathrm{E} / \mathrm{N}>$ $(\mathrm{E} / \mathrm{N})_{c}$ is no longer satisfied over a large enough region to sustain the discharge. At this point the discharge ceases and the current drops rapidly to zero. Another PD will not occur at this location until the surface and space charge has dissipated to restore the field to the level where electron-avalanche development in the gas becomes possible, or until the externally applied voltage is effectively increased. If a dc applied voltage is used, the PD activity can be completely quenched after a few pulses and may not resume for many hours; whereas for an ac applied voltage, PD activity will occur on alternate half cycles when there is field reversal.

The PD phenomenon is also inherently a stochastic process in which "memory effects" play an important role. The significance of memory in this phenomenon has been demonstrated recently (Van Brunt \& Kulkarni, 1990) for negative corona pulses that occur in air and other electronegative gases. The memory is associated with the effect of residuals such as surface charge, ions, or metastable species from previous pulses on the initiation and growth of subsequent pulses. As a consequence of these memory effects, the amplitudes and times-of-occurrence of subsequent pulses are not independent random variables. This means that the distributions in pulse amplitudes are not independent of distributions in pulse time separations, that is, there can be 

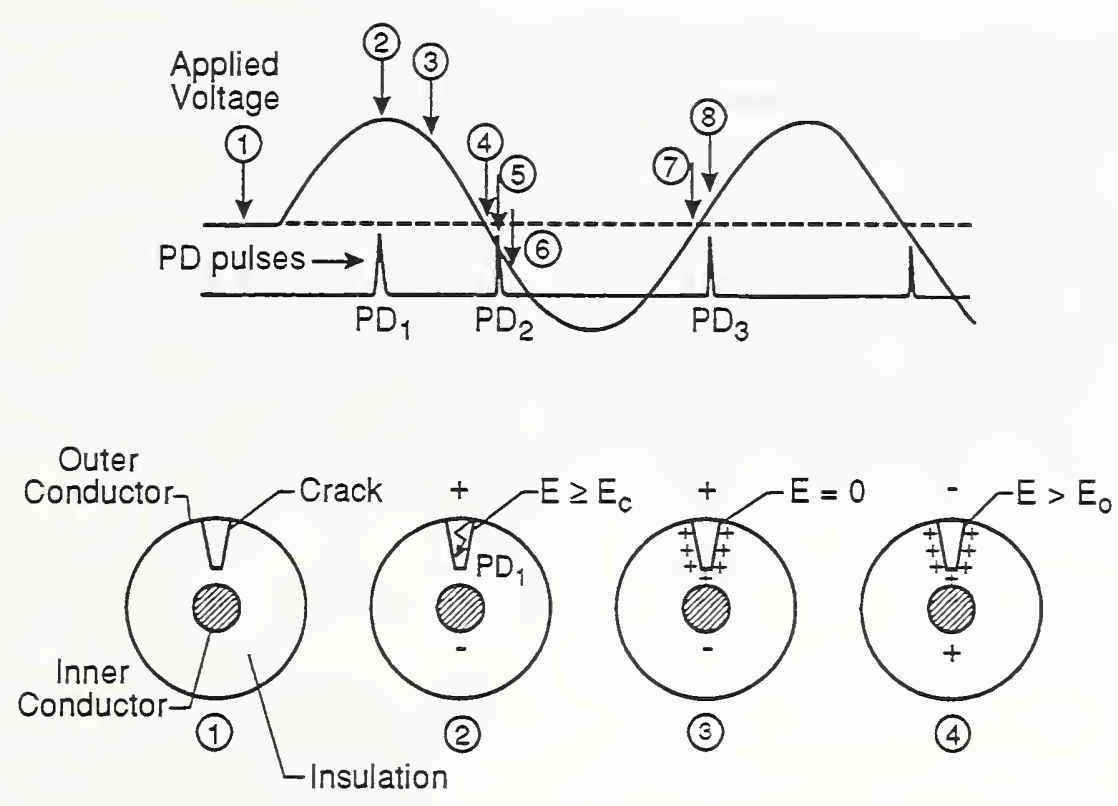

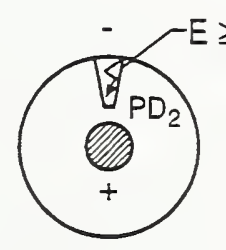

(5)

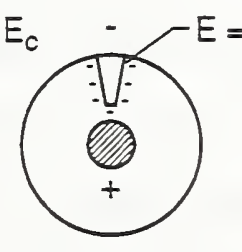

(6)

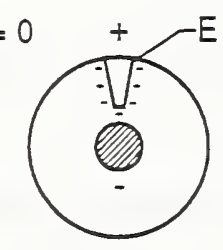

(7)

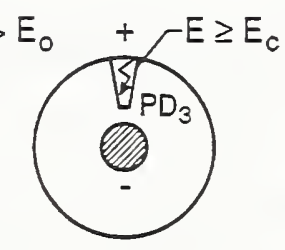

(8)

Figure 5-1. Diagrammic representation of an ac-generated PD process associated with a crack in the insulation of a cylindrically symmetric cable. Shown are the first PD pulse $\left(\mathrm{PD}_{1}\right)$ and successive pulses $\left(\mathrm{PD}_{2}, \mathrm{PD}_{3}, \ldots\right)$ that occur in the indicated phases of the applied voltage. In this figure, $\mathrm{E}$ represents the mean electric-field strength in the crack, $\mathrm{E}_{\mathrm{c}}$ is the critical value of $E$ required for onset of a $P D$, and $E_{0}$ is the value of $E$ at the applied voltage zero crossing.

significant correlations between the amplitude of any PD pulse and the amplitudes and time separations from previous pulses.

An example of the type of $P D$ behavior that can be anticipated in the case where a crack exists in a cylindrical cable is illustrated in figure 5-1. It is assumed that when a sufficiently high alternating voltage is applied to the cable, the electric field in the crack reaches a magnitude greater than $E_{c}$ at which a discharge can develop if initiated by a free electron. Assuming an availability of free electrons, it can be expected that the first discharge pulse will occur before or near the maximum in the first half-cycle when the voltage exceeds the inception level. This discharge pulse will cause charge to accumulate on the walls of the crack with a resulting decrease in the average field within the crack. The next PD pulse will not occur until the voltage changes sign so that the presence of the charge on the walls enhances the local field to a level sufficient to allow initiation and development of a discharge. Subsequent pulses will occur predominantly on the rising portion of each half cycle. This type of 
correlation of PD pulses with the phase of the ac voltage is a well known characteristic of discharges that occur in the presence of an insulating surface or dielectric barrier (Okamoto \& Tanaka, 1985; Hikita et al., 1990; Schifani, 1986).

The width of PD pulses typically lies in the range of 1 to $100 \mathrm{~ns}$ (Scott et al., 1987). If one uses a narrow-band pulse detection system or if it can be assumed that pulse shape does not vary significantly with amplitude, then it is possible to express the pulse amplitude in units of picocoulombs (Kreuger, 1965); the pulse amplitude becomes a measure of the total charge generated in the pulse as given by:

$$
q=\int_{\Delta \tau} i(t) d t
$$

where $i(t)$ is the instantaneous discharge current and $\Delta \tau$ is the time duration of the pulse. It might be expected that the mean amplitude of the PD pulses will be correlated with the size of the defect site in which the PDs occur, such as the size of the crack or void in a solid dielectric. However, because of the possible strong correlations among the amplitudes of successive PD pulses, caution must be exercised in making any inferences about defect size from observed PD amplitudes.

It should be emphasized that, because the generation of PDs depends on the presence of regions in the insulation that contain either a gas, or a readily vaporized fluid, their occurrence or lack thereof cannot provide indications of structural weaknesses or defects in the bulk of solid insulation that are not connected to gaseous regions. If PD pulses are observed to occur in cables above the background noise level and are also found to be highly correlated with voltage and/or reflections, then one can infer with some confidence that there exist defects connected to gaseous regions as might be assumed with voids or cracks. The absence of PD pulses, on the other hand, is not an indication of the absence of defects. Nevertheless, defects that give rise to detectable PD pulses are those that are most likely to be responsible for eventual failure by electrical breakdown.

The theory of partial discharge is in many respects still quite rudimentary. Only relatively recently has there been a successful prediction of one of the simplest types of PD phenomena, namely negative Trichel-type corona pulses in air or oxygen in a point-to-plane discharge gap (Morrow, 1985). However, even in this case, the theory can only give the magnitude and shape of the first Trichel pulse since it neglects the memory effects that must be considered in the development of subsequent pulses. The types of memory effects that need to be considered for this phenomenon have recently been investigated (Van Brunt \& Kulkarni, 1990). Memory effects have also been shown to be important for dielectric-barrier type discharges (Van Brunt \& Cernyar, 1991; Kulkarni et al., 1990; Van Brunt et al., Influence of a dielectric barrier, 1991).

In the cases where PDs are formed in the presence of solid dielectric surfaces, the theory of partial discharge becomes more complicated due to the necessity of including the effects of surface charging. Nevertheless, it has been shown that it is possible to 
make "reasonable" estimates of PD magnitudes using scaling laws and semiempirical models for the cases of ellipsoidal voids (Crichton et al., 1988; Pedersen, 1987; Crichton et al., 1989) and parallel plate type dielectric barriers (Egli \& Eliason, 1989; Eliasson \& Kogelschatz, 1989). It has been predicted that the magnitude of PDs in voids will depend significantly on the orientation and size of the void as well as on the type of gas present in the void. Previous attempts to predict PD onset based on calculations of electric stresses in gaseous cavities in solid dielectrics (Morrow, 1985; Crichton et al., 1989) are generally limited to the first pulse since these calculations neglect modifications of the discharge environment by previous discharge pulses. Once $\mathrm{PD}$ activity commences within a gas-containing dielectric cavity, surface charge can become quasi permanent, that is, it dissipates in a time long compared to the time between PD pulses, so that one's ability to predict the onset of subsequent pulses becomes limited by one's knowledge of the PD deposited surface charge distribution.

\subsection{Measurement of partial discharges (general)}

The available techniques for detecting PDs and for quantitative measurement of their magnitudes have been covered in recent literature (Van Brunt et al., Recent advances, 1992; Kreuger, 1965; Bartnikas, 1987; Bartnikas, 1990; Boggs \& Stone, 1982; Boggs, 1990; Steiner, 1991) and will not be repeated here. The purpose of this section is to point out the various properties of PDs that can be measured and what it is that can be learned from such measurements in the light of our understanding of the physics of the phenomenon. The measurement of partial discharge is commonly used for insulation certification in the manufacturing of electrical apparatus and for evaluation of insulation performance and integrity during operation of high-voltage equipment. Notwithstanding the progress that has been made over the past years in achieving a better understanding of the various mechanisms that may precipitate failure in high-voltage insulating systems, PD measurements have retained their preponderant importance in the area of electrical-insulation evaluation. This importance must be largely attributed to the fact that defects, introduced into the insulation systems during various manufacturing steps, often involve macroscopic cavity inclusions which can be detected readily by means of routine tests with the tacit assumption that such cavities lead to ionization and subsequent pulse-type discharge in order to activate the response of conventional PD detectors.

As noted in the previous section, the pulsating PD phenomenon of interest to us here is a stochastic process, which, although not totally random, exhibits significant statistical fluctuations in amplitude and in time of occurrence. It is, therefore, generally not possible to define a single, well determined amplitude for PDs that occur at a particular location in the insulating medium. Partial discharges can only be quantified in terms of statistical distributions and corresponding averages. 
Commercially available PD measurement systems yield rather limited information about the PD phenomenon; they often give only the maximum or mean PD pulse amplitude or pulse repetition rate or both. There are, however, recently developed measurement systems that also provide data on the statistical distributions of pulse amplitudes (Bartnikas, 1987) and the correlations of pulse occurrence times and amplitudes with the phase of an applied ac voltage. Certainly, having a knowledge of these statistical distributions allows a more precise assessment of the type of incipient fault with which the observed PDs are associated than is possible with only knowledge about average values. Nevertheless, it has been shown (Van Brunt \& Kulkarni, 1990; Van Brunt \& Cernyar, 1991) that because of memory effects that give rise to correlations among successive pulses, the PD pulse-amplitude and time-separation distributions may not be independent. This situation adds to the complexity of the interpretation of these distributions. If the discharge pulses occur predominantly from a single site, then measurement of phase restricted conditional pulse-amplitude and time-separation distributions can aid in the interpretation of the data (Van Brunt \& Cernyar, 1991). Unfortunately, methods for measuring conditional distributions are still in the developmental stage and have not yet been incorporated into commercially available instruments. For cases where PDs occur randomly from many different sites in the insulating system, it even becomes difficult to interpret conditional distributions. This difficulty can often be partly overcome for cables by using the PD analysis discussed in Section 6 . With this method it is possible to determine approximate $\mathrm{PD}$ locations by making correlations of $\mathrm{PD}$ pulse signals with reflected signals (Steiner, 1991; Steiner, 1988; Mashikian et al., 1989; Steiner \& Martzloff, 1990). However, this approach is also not a standard feature of commercially available PD measurement systems, and one is forced to set up special circuits for such measurements, as described in Section 7 .

The measurement of time correlations for PD pulses requires the use of relatively broad-band detection electronics, which, although readily available, are not commonly used and therefore considered to be somewhat nonstandard. With broad-band detection it is also possible under some conditions to acquire detailed information about PD pulse shapes that can be interpreted in terms of the basic physical mechanisms associated with the PD phenomenon (Morrow, 1985). Pulse-shape measurements are difficult to perform for practical insulation configurations and consequently the usefulness of such measurements for diagnostic purposes has yet to be proven.

The occurrence of partial discharges is associated with electromagnetic, optical, and acoustic emissions from the discharge site. Although radio-frequency, optical and acoustic detectors have been used successfully to locate PDs in high-voltage substation equipment (Bartnikas, 1987) such as in transformers, capacitors, and switchgear, such detection methods have limited usefulness for insulated cables, especially if the cables are not physically accessible. In the case of enclosed cables, one is generally restricted to electrical detection methods. 


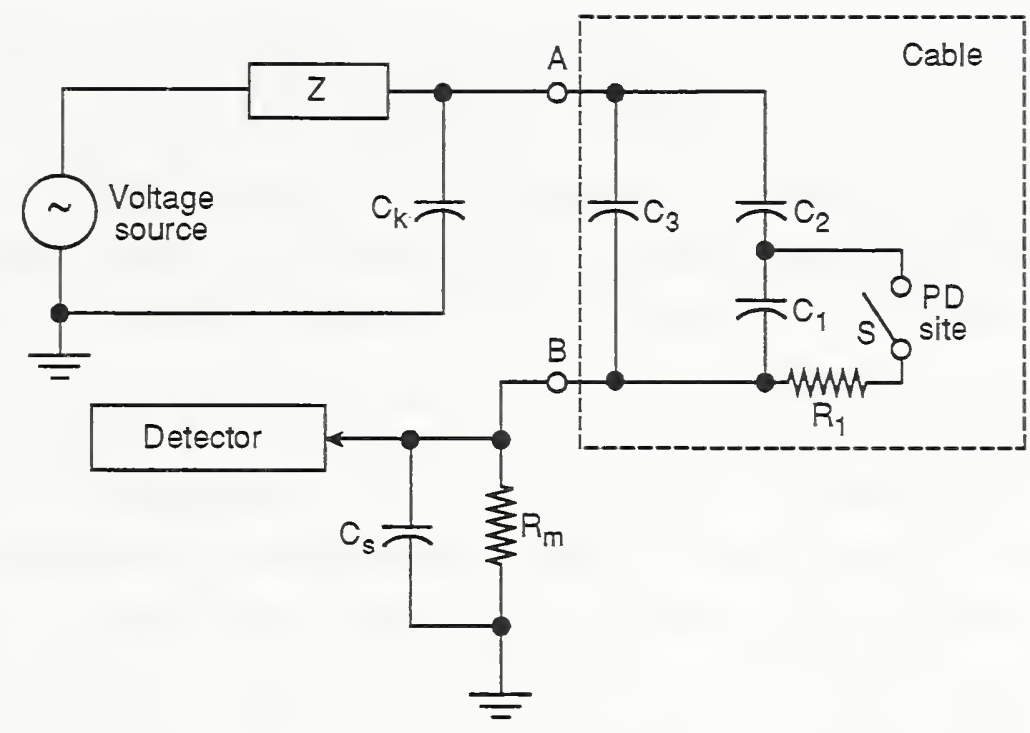

P.D S-2

Figure 5-2. Equivalent circuit of a PD detection system applied to an open-ended cable. The $\mathrm{PD}$ site is simply represented by a switch $\mathrm{S}$ that discharges the capacitor $\mathrm{C}_{1}$ through resistance $\mathrm{R}_{1}$ upon closing, thereby simulating the approximate behavior of an internal PD.

The sensitivity of PD detection by electrical means in cables can be significantly affected by the cable length and configuration as well as by the environment in which the cable is placed. The PD detection sensitivity is also affected by the external circuit to which the cable is connected. This is illustrated by the simplified PD test circuit shown in figure 5-2. The cable in this case is represented by the equiralent circuit between points $\mathrm{A}$ and $\mathrm{B}$. Here, $\mathrm{C}_{1}$ is the capacitance associated with a small fault (crack or cavity) which is in series with $\mathrm{C}_{2}$. The fault-free cable is assumed to have a capacitance $\mathrm{C}_{3}$ such that $\mathrm{C}_{3} \gg \mathrm{C}_{1} \gg \mathrm{C}_{2}$. The occurrence of a $\mathrm{PD}$ in the carity can be represented by the closing of switch $S$ which discharges $C_{1}$ through $R_{1}$. The amount of charge $q$ transported in the cavity during the $P D$ is given by $q=\Delta V_{1} C_{1}$, where $\Delta V_{1}$, is the resulting voltage drop across $C_{1}$ when $S$ is closed. The resulting voltage drop $\Delta V_{A B}$ across the cable is given by

$$
\Delta V_{A B}=\frac{C_{2}}{C_{2}+C_{3}} \Delta V_{1}=\frac{C_{2} q}{\left(C_{2}+C_{3}\right) C_{1}} .
$$

From this relationship it can be seen that the larger the capacitance of the cable, the smaller will be the observable signal from a discharge of a given magnitude $q$.

As seen in figure $5-2$, the cable is connected to a voltage source with coupling capacitor $C_{k}$ and the current $i(t)$ from the $P D$ is sensed across a resistance $R_{m}$ with a stray capacitance $\mathrm{C}_{s}$ to ground. Assuming a short-duration PD pulse that can be represented by a Dirac delta function $\delta(t)$, it can be shown that the corresponding instantaneous voltage $V_{m}(t)$ across $R_{m}$ due to the PD is given approximately by

$$
V_{m}(t) \simeq \frac{\mathrm{C}_{3} \Delta V_{A B}}{\mathrm{C}_{\mathrm{s}}+\mathrm{C}_{3}\left(1+\mathrm{C}_{s} / \mathrm{C}_{k}\right)} \exp (-t / \tau),
$$


where

$$
\tau \simeq R_{m}\left(\mathrm{C}_{s}+\frac{\mathrm{C}_{3} \mathrm{C}_{k}}{\mathrm{C}_{3}+\mathrm{C}_{k}}\right)
$$

The voltage $V_{m}(t)$ at the measurement point is thus a fast rise (ideally a voltage step) followed by an exponential decay with a time constant $\tau$. The original PD pulse generated in the cavity $\mathrm{C}_{1}$ can thus be severely deformed by the external circuit.

The important message to be derived from the above analysis is that the sensitivity for PD pulse detection in cables can vary significantly from cable to cable. The detection sensitivity depends, for example, on cable length, diameter, and whether or not the cable has an outer grounded shield. If the cable is not encased in a shield, the possibility for forming detectable PDs will depend on proximity of the cable to grounded conductors. Unshielded cables that are located far from other conductors may require very high voltages for initiation of PDs. It is important to note that PD activity can occur in cables independent of whether or not they are shielded. Because the occurrence of PDs in unshielded cables may require voltages significantly above the rated value, it can be argued that defects in such cables with which PDs are associated are likely to be of little or no relevance in assessing the reliability of the cable, that is, its resistance to breakdown under normal stress will not be affected by the presence of such defects, but the cable can still become a liability under loss of coolant accident (LOCA) conditions.

\subsection{Partial discharge-induced degradation of insulation}

When attempts are made to evaluate the integrity of an insulation system by applying sufficiently high voltages to cause deliberately the onset of corona or partial discharge, it becomes necessary to consider the effects of such discharges in enhancing potential fault locations, for instance, via chemical decomposition of the insulation. The subject of degradation of insulation by partial discharge is one that has received considerable attention for obvious reasons (Tanaka, 1986; Goamez-Garcia et al., 1990; Mason, 1978). Any type of PD phenomenon can have the effect of changing the physical and chemical properties of the insulation at a fault location and these changes can, in turn, affect the characteristics of the PDs at this location. If PD induced changes in the insulation enhance the rate of energy deposition by PDs, then it becomes possible that this type of feedback effect can, beyond a certain point, lead to a very abrupt transition to electrical breakdown.

The deterioration of insulation at a PD site comes about through erosion by ion on electron bombardment and chemical degradation by active gases generated in the discharge. Even when the energy deposition by individual PDs is quite small, (below a micro joule), some significant fraction of the electrons produced in the discharge 
can have energies in excess of $10 \mathrm{eV}$ (Van Brunt, 1987; Mason, 1978; Daris, 1984). These relatirely high-energy electrons can break or disrupt molecular bonds upon impact with a surface since bond strengths are typically below $10 \mathrm{eV}$, such as, $3.5 \mathrm{eV}$ for $\mathrm{C}-\mathrm{H}$ and $6.2 \mathrm{eV}$ for $\mathrm{C}=\mathrm{C}$. The presence of reactive species like atomic oxygen and $\mathrm{O}_{3}$ produced during the $\mathrm{PD}$ can also induce chemical changes in some materials. Even in static air, accumulation of $\mathrm{O}_{3}$ can initiate cracks in elastomers, polymers, and varnishes.

There have been studies of insulation degradation induced by PD activity; particularly for high-voltage applications in which the occurrence of PDs can be expected under normal operating conditions (Mason, 1978). Most of the studies indicate that aging of insulation due to PDs is a very slow process provided the system in which the insulation resides is not significantly orerstressed. Serious weakening of the insulation leading to a rapid breakdown generally only occurs when PDs are initiated at sites that are already seriously damaged by preriously introduced defects or stress. The initiation of PDs for relatively short periods of time associated with moderate orerstressing of cables for test purposes is therefore not likely; under normal circumstances, to result in a significant weakening of the insulation. Cables are generally designed to accept orerstressing without risk of failure. It should be kept in mind, howerer, that partial discharges and breakdown processes are stochastic phenomena (Dissado, 1990) so that it can never be assumed with $100 \%$ certainty that orerstressing will not result in breakdown or affect future reliability of the cable. There is always a small level of risk associated with the orerstressing of cables, and unfortunately it is not possible to assess quantitatively this risk for all conceivable cables or cable configurations. Details of the physical and chemical processes that occur in all types of solid materials needed to make reasonable predictions about failure probabilities are simply not known. Although there is now a considerable amount of research activity underway to investigate $P D$ phenomena, much of the information obtained is quite empirical and restricted to particular test configurations which may or may not be relevant to real-cable configurations. 


\section{Partial Discharges in Cables}

This chapter develops the theoretical aspects of partial discharge (PD) measurement in cables. The partial discharge analysis (PDA) method requires a thorough understanding of the measurement process in terms of the received signals and the methods used to extract the pertinent parameters. From a fundamental point of view, one is only concerned with deciding whether a harmful PD site is located within the cable. This is not as simple as it seems because there are other innocuous PD sites within the cable and exterior to it. The presence of partial discharges exterior to the cable requires that the position of the PD site be determined so only those partial discharges internal to the cable are used in the analysis. Sorting of the partial discharges according to their position allows for statistical analysis of the individual PD sites.

\subsection{Nature of the measured signals}

To make a judgment whether a particular partial discharge is associated with a defect in the cable insulation, it is necessary to estimate the parameters of each $\mathrm{PD}$ pulse generated by ionization at the various sites. As mentioned in Section 5, the partial discharges can be represented by a stochastic random point process. Three fundamental quantities are required to characterize the point process: the amplitude of the pulses, their times of occurrence and the positions in the cable where they are generated. During the course of the measurement, only a single PD pulse is characterized at a time. Unfortunately, when a sample is taken, it not only contains the PD pulse but other signals which corrupt the measurement. These interfering signals which corrupt the measurement must be dealt with in an appropriate manner. The purpose of this section is to characterize the measured signals seen by the instrument.

As described in Section 5, the PD signal is a pulse and the shape of the pulse is determined by the propagation characteristics of the cable being measured. At its point of origin, the PD pulse is very narrow, which means that it has frequency components that can extend into the hundreds of megahertz range. As described in Section 6.2, typical cables bandlimit the PD signal. By the time the pulse arrives at the receiver, it has lost most of its high-frequency content, resulting in a usable bandwidth typically less than $10 \mathrm{MHz}$. To optimize the measurement, all of the available bandwidth needs to be utilized. With the measurement bandwidth still $10 \mathrm{MHz}$, this measurement is considered to be broadband. Measurements with this bandwidth are subject to a variety of interference and noise.

The amplifiers used to condition and amplify the PD signals are not ideal, they generate noise. This noise is thermally generated in the amplifier components and is 
accurately modeled as a Gaussian random process. The bandwidth of the amplifiers is larger than the bandwidth of the received signal and hence the thermal noise is much wider in bandwidth than the PD signal. With respect to the PD pulse, the thermal noise in the amplifiers is considered to be white noise. The term "white" means that the frequency domain power spectrum of the noise is constant for all frequencies. In the frequency domain the noise is described as

$$
N(\omega)=N_{0}
$$

where $N(\omega)$ is referred to as the power spectral density of the noise and $N_{0}$ is the noise constant. To limit unwanted interference outside of the bandwidth of the partial discharge, a filter has been incorporated which also limits the bandwidth of the white noise. The filter has a frequency domain response, $H_{e}(\omega)$, which modifies the thermal noise so that the power spectral density of the noise becomes

$$
N(\omega)=N_{0}\left|H_{e}(\omega)\right|^{2}
$$

In terms of the measurement, the received signal is now corrupted by filtered white noise.

The hardware implementation of the PDA method is all-digital which means that the received signals are quantized by an analog-to-digital converter. The process of quantization distorts the measured signal and this distortion is often modeled as additive noise. While this is only an approximation, results based on this assumption are quite good. Since there is a small amount of thermal noise present in the unquantized signal, the additive term due to the quantization is adequately modeled as a uniform white noise process. This white noise term is not modified by any filtering and in terms of the measurement appears as a white noise term. The noise corrupting the signal is now given by

$$
N(\omega)=N_{0}\left|H_{e}(\omega)\right|^{2}+N_{Q}
$$

where the new term, $N_{Q}$, is the quantization noise constant. For many signal processing algorithms, if noise is present, whiteness is a desirable property.

Another type of noise that corrupts the measurement is more aptly labeled as interference. This noise has a continuous nature, which means that it is constantly present, and will be referred to as continuous noise or interference. This type of interference is normally caused by radio broadcasts and is predominantly in the AM broadcast band. This type of noise can also be generated by the digitizer or computer if precautions are not taken to shield the instrumentation properly. This noise can couple into the system in different ways. One primary coupling path is through the cable termination at the instrumentation end. This noise path can be minimized by shielding the high-voltage coupling capacitor as in the PDA system. However, due to the nature of the high voltage, complete shielding is diffcult to implement. Another primary coupling path is along the length of the cable itself. If the polarization of the 
incident field is oriented properly, voltages and currents are induced in the cable and propagate along it. Depending on the proximity of the source of the interference to the cable, these signals can be quite large, especially in unshielded cables. Typically, these interfering signals are narrow-band and only corrupt a portion of the frequency band occupied by the PD signal. In terms of the noise model, this new term is also additive

$$
N(\omega)=N_{0}\left|H_{e}(\omega)\right|^{2}+N_{Q}+N_{c}(\omega)
$$

where $N_{c}(\omega)$ is the continuous noise term which may be sharply peaked at certain discrete frequencies. This type of interference has certain characteristics that can be used to mitigate its influence. Narrow-band broadcasts are adequately modeled by Gaussian random processes which means that they can be completely characterized by their power spectral density. This property opens the avenue for the instrument to use a variety of nearly optimal signal processing algorithms to reduce the influence of the noise.

Another type of noise is also present and is referred to as impulsive noise. This type of noise is intermittent and usually quite strong. It is generated by a variety of sources and is usually associated with some type of switching operation such as thyristor switching, motor starting, or switch closing. This type of noise is usually so strong that it swamps out other signals so there is often no possibility of retrieving the desired signal. Impulsive interference couples into the cable system in the same way as continuous interference. While this noise is overwhelming when present, the fortunate aspect is that it usually appears sporadically. Impulsive type interference can be modeled as a stochastic point process in the same manner as partial discharge. This noise term can be added to the noise model as an ac-coupled, marked, filtered Poisson point process:

$$
N(\omega)=N_{0}\left|H_{e}(\omega)\right|^{2}+N_{Q}+N_{C}(\omega)+\lambda_{I} \overline{I^{2}}\left|H_{I}(\omega)\right|^{2} .
$$

The intensity parameter $\lambda_{I}$ describes the number of impulsive events per second that disturb the measurement. The mean square value of the marks, $\overline{I^{2}}$, describes the amplitude characteristics of the impulses. The filter, $H_{I}(\omega)$, describes the shape of the impulse and is determined by the electromagnetic characteristics of the source generating the impulse and the manner in which it is coupled into the cable. The amplitudes of the impulses are usually very large but the count rate is typically very low, which means that it does not present much of a problem when detecting partial discharges. The easiest way to eliminate the effect of this interference is to eliminate records containing it.

As mentioned in Section 5, partial discharge is a stochastic point process and to process these signals a model is needed. The simplest model that can be used for the noiseless received signal, $s(\mathrm{t})$, is a marked filtered Poisson point process. This model is

$$
s(t)=\sum_{i=1}^{N_{T}} a_{i} \delta\left(t-t_{i}\right) * h_{c}(t)
$$


where $N_{T}$ is the number of pulses in the time interval $(0, T)$ and the symbol * denotes a convolution. The partial discharges are modeled as impulses that occur at times $t_{i}$. Each impulse has a amplitude $a_{i}$ and the impulses are convolved with pulse shape, $h_{c}(t)$, determined by the cable propagation characteristics. The power spectral density of this process is given by

$$
|S(\omega)|^{2}=(\bar{a})^{2} \lambda^{2} H_{c}^{2}(0)+\overline{a^{2}} \lambda^{2}\left|H_{c}(\omega)\right|^{2}
$$

The first term on the right hand side is a dc term that is usually not observed because the measurements are ac coupled. The term $\lambda$ is the intensity of the process and describes the number of partial discharges per second. This intensity parameter, $\lambda$, can be interpreted in terms of a probability for the occurrence of a discharge pulse within an arbitrarily small time interval $\Delta$ t, i.e.,

$$
\operatorname{Pr}\left\{N_{\Delta t}=1\right\}=\lambda \Delta t+o(\Delta t)
$$

where the term $o(\Delta t)$ is a small higher order term defined so that $o(\Delta t) / \Delta t$ goes to zero as $\Delta t$ goes to zero.

Another mark can be added to the description to identify the position within the cable where the partial discharge originates. This addition is accomplished by including the term

$$
s(t)=\sum_{i=1}^{m} \sum_{j=1}^{N_{T, z_{i}}} a_{j, z_{i}} \delta\left(t-t_{i j}\right) * h_{c}\left(t ; z_{i}\right)
$$

in Eq. 6-9, where the same definitions used above apply but for the specific set of locations $z_{i}$ and corresponding occurrence times $t_{i j}$. In Eq. 6-9, $m$ represents the number of cable defect sites.

The final type of interference encountered in the measurements is the PD process itself. If two distinct PD pulses occur within a time that is less than twice the round trip propagation delay of the cable under test, then the measurement record will contain both pulses. When two distinct PD pulses are included in the measurement, they cause an error. Since partial discharge is a stochastic point process, events occur randomly in time. Fortunately, the measurements are made at voltages slightly above the $\mathrm{PD}$ inception so the $\mathrm{PD}$ process at any one site is sparse. In this case, sparse means that the probability that a particular site will generate two distinct PD pulses in close temporal proximity is small. If the process is modeled as a Poisson process, then the probability that more than one partial discharge occurs at the site is

$$
\operatorname{Pr}\left\{N_{T, z_{i}}>1\right\}=1-\exp \left(-\lambda_{z i} T\right)
$$

where $T$ is the length of the record and $z_{i}$ is the position of the PD site. This probability is normally so small that this type of event will usually not be observed during the course of a measurement. 


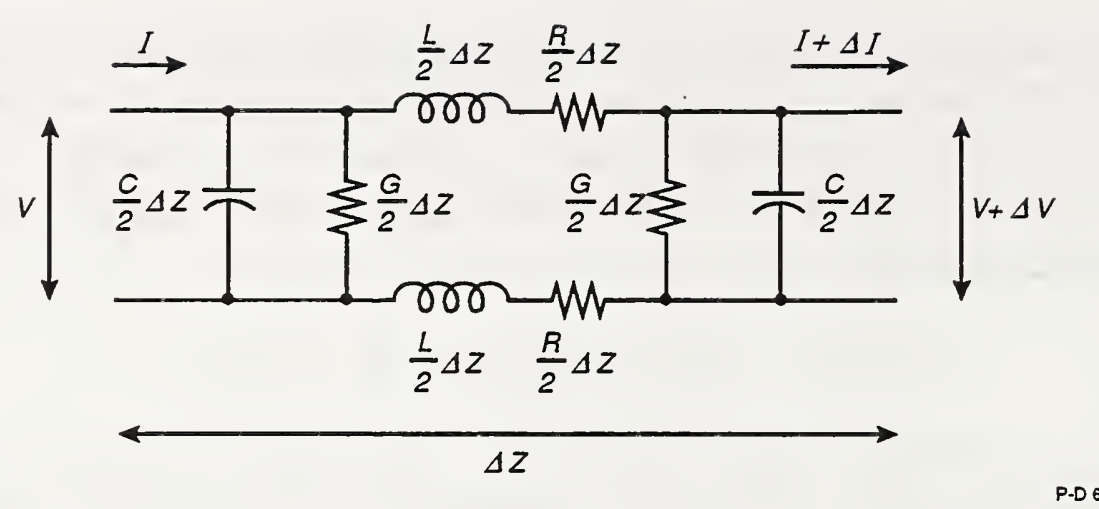

Figure 6-1. Elementary circuit diagram for cable

When the cable has multiple PD sites, this situation increases the probability that two distinct $\mathrm{PD}$ pulses may be observed in any one record. Furthermore, as the excitation voltage is increased above the inception voltage, the probability of multiple events increases. If the $\mathrm{PD}$ process is modeled as the superposition of $M$ Poisson processes generated by partial discharges occurring at $m$ sites, the probability of observing multiple events in a record is given by

$$
\operatorname{Pr}\left\{N_{T}>1\right\}=1-\exp \left(-\sum_{i=1}^{m} \lambda_{z_{\mathrm{i}}} T\right)
$$

This probability is significant enough that a few of these events will be recorded during a measurement. Methods for eliminating the errors due to multiple events in a record are discussed in Section 6.4.2.

The preceding discussion highlights the details of the interference and noise that limit the detectability of partial discharge. The detectability of partial discharge is also intimately related to the characteristics of the PD pulse. The following sections give some of the theoretical details about the limitations of the measurements and methods used to maximize the performance of the PDA method.

\subsection{Cable propagation}

Fundamental to the methods used to locate partial discharges in cables is the modeling of the pulse propagation along the conductors. A thorough understanding of the propagation in cables is necessary in order to effectively design algorithms to estimate the parameters of interest. The following descriptions provide an overview of the solutions necessary for analyzing PD pulses in cables.

The cable can be modeled as a distributed circuit where the cable is thought of as a cascade of elemental lengths, each consisting of an electrical circuit. The electrical circuit of the elemental length, $\Delta Z$, is shown in figure 6-1. It consists of the series resistance of the conductors, the series inductance associated with the conductors and 


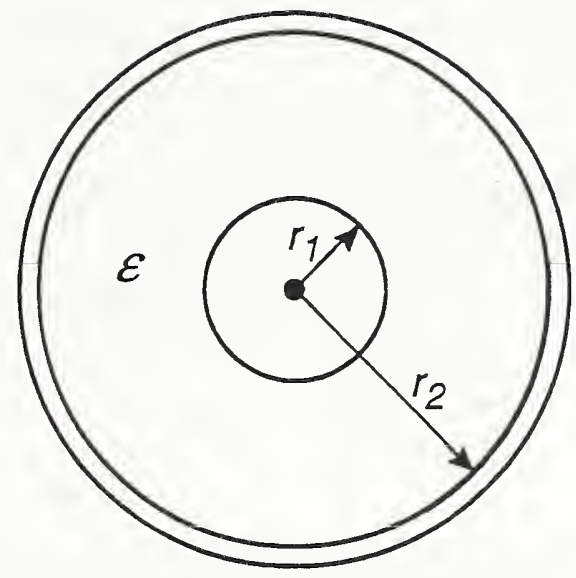

P.D 6-2

Figure 6-2. Cross-section of coaxial cable

their configuration, the capacitance between the conductors, and the conductance of the insulation between the conductors. $R, L, C$, and $G$ are, respectively, the per unit length values of the resistance, inductance, capacitance, and conductance of the cable. These parameters are determined from field theory or measurement, and include such phenomena as skin effect, temperature and frequency dependence of the material properties. They are dependent on sizes and configurations. Two specific transmission lines are of interest: the coaxial line, a shielded structure and the parallel wire line, an unshielded structure. For the coaxial line, shown in cross section in figure 6-2, the parameters are

$$
\begin{aligned}
C & =\frac{2 \pi \epsilon}{\ln r_{2} / r_{1}} \\
G & =\frac{2 \pi \sigma_{2}}{\ln r_{2} / r_{1}} \\
R & =R_{\text {inner }}+R_{\text {outer }} \\
& \approx \frac{1}{2 \pi}\left(\frac{1}{r_{1}}+\frac{1}{r_{2}}\right)\left(\frac{\rho_{1} \omega \mu_{1}}{2}\right)^{\frac{1}{2}} \\
L & \approx \frac{1}{2 \pi}\left[\mu_{2} \ln \frac{b}{r_{1}}+\left(\frac{1}{r_{1}}+\frac{1}{r_{2}}\right)\left(\frac{\rho_{1} \mu_{1}}{2 \omega}\right)^{\frac{1}{2}}\right]
\end{aligned}
$$

where the material constants are defined as:

- $\epsilon$ is the permittivity of the insulation.

- $\sigma_{2}$ is the conductivity of the insulation.

- $\rho_{1}$ is the resistivity of the conductor.

- $\mu_{1}$ is the permeability of the conductor. 


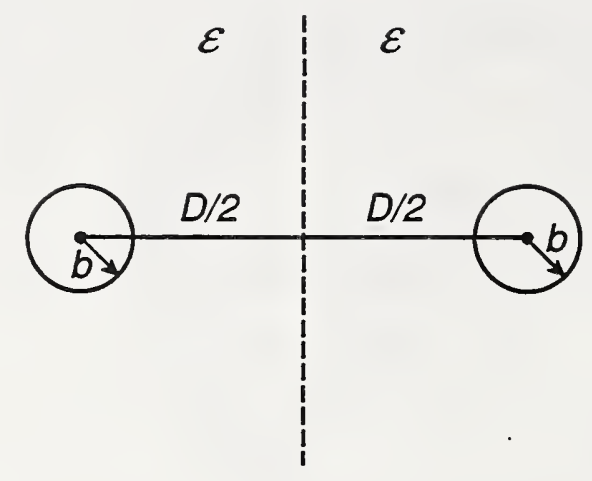

P.D 6-3

Figure 6-3. Cross-section of two-conductor cable

- $\mu_{2}$ is the permeability of the insulation.

- $\omega$ is the angular frequency.

For the parallel wire line, shown in cross section in figure 6-3, the parameters are

$$
\begin{aligned}
C & =\frac{\pi \epsilon}{\cosh ^{-1} D / 2 b} \\
G & =\frac{\pi \sigma}{\cosh ^{-1} D / 2 b} \\
R & =\left(\frac{\rho_{1} \omega \mu_{1}}{2}\right)^{\frac{1}{2}} \frac{1}{\pi b} \\
L & =\left(\frac{\rho_{1} \mu_{1}}{2 \omega}\right)^{\frac{1}{2}} \frac{1}{\pi b}+\frac{\mu_{2}}{\pi} \cosh ^{-1} D / 2 b
\end{aligned}
$$

For the frequencies of interest and in the case of the coaxial transmission line, the approximations are generally very good. For the parallel wires, the approximation is reasonable only if the following assumptions are valid. First, the wires are assumed to be surrounded by a uniform dielectric. For the cables of interest this is an idealization because the dielectric is a composite of the wire insulation, the filler material, the jacket, and the surrounding environment. Secondly, nearby conductive structures are ignored, that could in some cases significantly affect the parameters of the model.

The parameters $C, G, R$, and $L$ are necessary for determining the propagation constants for the models. The equations for wave propagation are found by applying Kirchoff's laws to the elemental circuit model in Figure 6-1 (Weeks, 1964). If the cable is terminated in its characteristic impedance, then the equations describing the voltage and current waves propagating along the cable are

$$
V(\omega)=V_{0} e^{-\gamma(\omega) z}
$$

and

$$
I(\omega)=\frac{V_{0}}{Z_{0}} e^{-\gamma(\omega) z}
$$


where $V_{0}$ is the voltage exciting the cable and $z$ is the position along the cable. The propagation factor, $\gamma(\omega)$, is given by

$$
\gamma(\omega)=\sqrt{(R+j \omega L)(G+j \omega C)}
$$

and the characteristic impedance, $Z_{o}$, is given by

$$
Z_{0}=\sqrt{\frac{R+j \omega L}{G+j \omega C}}
$$

The propagation factor, $\gamma(\omega)$, is usually separated into two parts, one describing the attenuation of the signal and the other describing the velocity at which the signal travels. The attenuation, $\alpha(\omega)$, is given by

$$
\alpha(\omega)=\operatorname{Real}(\sqrt{(R+j \omega L)(G+j \omega C)})
$$

and the phase factor $\beta(\omega)$, given by

$$
\beta(\omega)=\operatorname{Imag}(\sqrt{(R+j \omega L)(G+j \omega C)})
$$

Equations 6-20 and 6-21 describe waves traveling only in the positive $z$ direction because there are no reflections from the ends of the cable.

Propagation in only one direction on a cable with a matched impedance termination is not adequate for analyzing a partial discharge pulse. In the general case, the PD pulse is generated within the interior of the cable and travels in both directions along the cable that is not terminated with matched impedances. A partial discharge can be modeled as a point current source exciting the cable at some interior location. A current source is a useful model since it does not cause an impedance discontinuity at the point of injection. To determine the equations for propagation consider a point current source, $I$, located at a position $z=x_{i}$ exciting a transmission line of length $L$ as in figure 6-4. This transmission line has a characteristic impedance $Z_{0}$ and is terminated by arbitrary impedances $Z_{n}$ at the near end and $Z_{f}$ at the far end. There are two solutions for pulse propagation generated by an interior current source. The two solutions apply to the respective regions on either side of the current source. The Fourier transform of solutions for the respective regions are: 


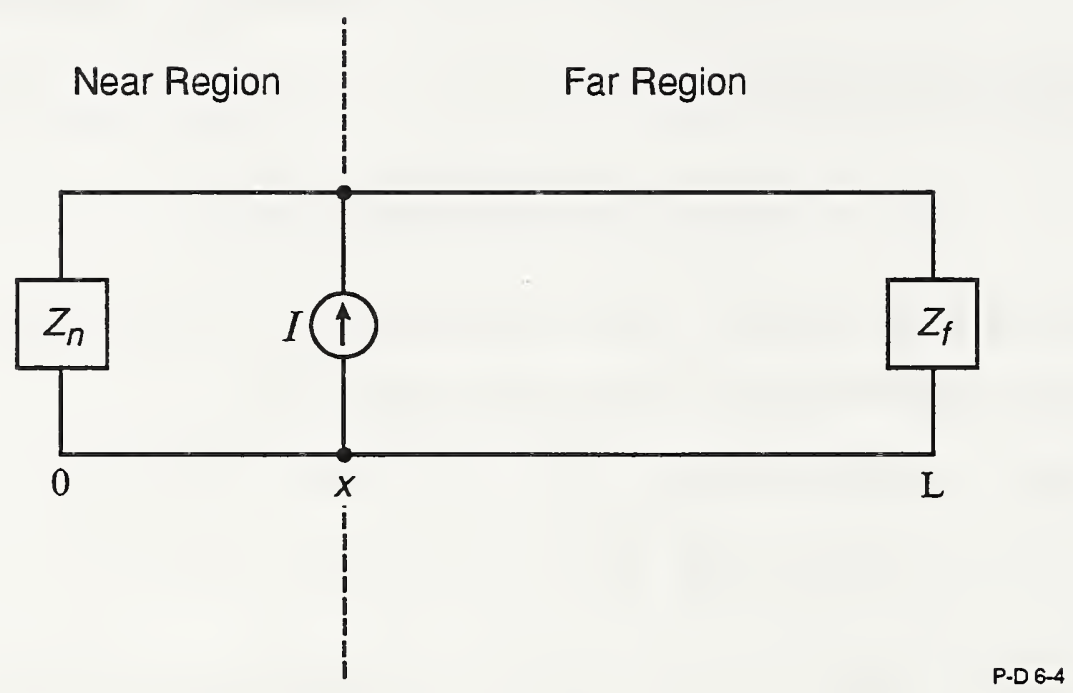

Figure 6-4. Terminated transmission line with PD current source 
Near Region

$$
V_{n}(\omega, z)=\frac{I(\omega) Z_{0}(\omega)\left[1+\rho_{f}(\omega) e^{2 \gamma(\omega)\left(x_{i}-\mathrm{L}\right)}\right]\left[e^{\gamma(\omega)\left(z-x_{i}\right)}+\rho_{n}(\omega) e^{-\gamma(\omega)\left(z+x_{i}\right)}\right]}{2\left[1-\rho_{n}(\omega) \rho_{f}(\omega) e^{-2 \gamma(\omega) \mathrm{L}}\right]}
$$

Far Region

$$
V_{f}(\omega, z)=\frac{I(\omega) Z_{0}(\omega)\left[1+\rho_{n}(\omega) e^{-2 \gamma(\omega) x_{i}}\right]\left[e^{-\gamma(\omega)\left(z-x_{i}\right)}+\rho_{f}(\omega) e^{-\gamma(\omega)\left(z+x_{i}-2 L\right)}\right]}{2\left[1-\rho_{n}(\omega) \rho_{f}(\omega) e^{-2 \gamma(\omega) L}\right]}
$$

The term, $I(\omega)$, is the Fourier transform of the PD current pulse exciting the cable. The quantities $\rho_{r}(\omega)$ and $\rho_{f}(\omega)$ are the voltage reflection coefficients at the ends of the cable defined, for the near end by

$$
\rho_{n}(\omega)=\frac{Z_{n}(\omega)-Z_{0}(\omega)}{Z_{n}(\omega)-Z_{0}(\omega)}
$$

and, for the far end, by

$$
\rho_{f}(\omega)=\frac{Z_{f}(\omega)-Z_{0}(\omega)}{Z_{f}(\omega)-Z_{0}(\omega)}
$$

Some new features are included in this solution: frequency-dependent parameters. In general, the characteristic impedance of the cable is a function of frequency as are the termination impedances. The propagation parameter is also, in general, a function of frequency. The frequency-dependent attenuation factor, $\alpha(\omega)$, determines the lowpass characteristics of the cable and is responsible for the high frequency losses in the cable. The frequency-dependent phase factor, $\beta(\omega)$, determines the propagation velocity of the cable. If the phase factor is a linear function of frequency, then the cable is considered to be a linear phase channel. Usually the phase factor deviates from this ideal behavior and the cable is considered to be dispersive. In a dispersive medium different frequencies travel at different velocities. A pulse traveling through a dispersive channel will become distorted even in the absence of any frequencydependent attenuation losses.

The equations for the two regions provide a complete solution including all reflections. Typically, one is only concerned with the first few reflections since the instrument only uses these. To convert the complete solution into an approximate solution one can perform a series expansion of the equations to retrieve the first few reflections. 


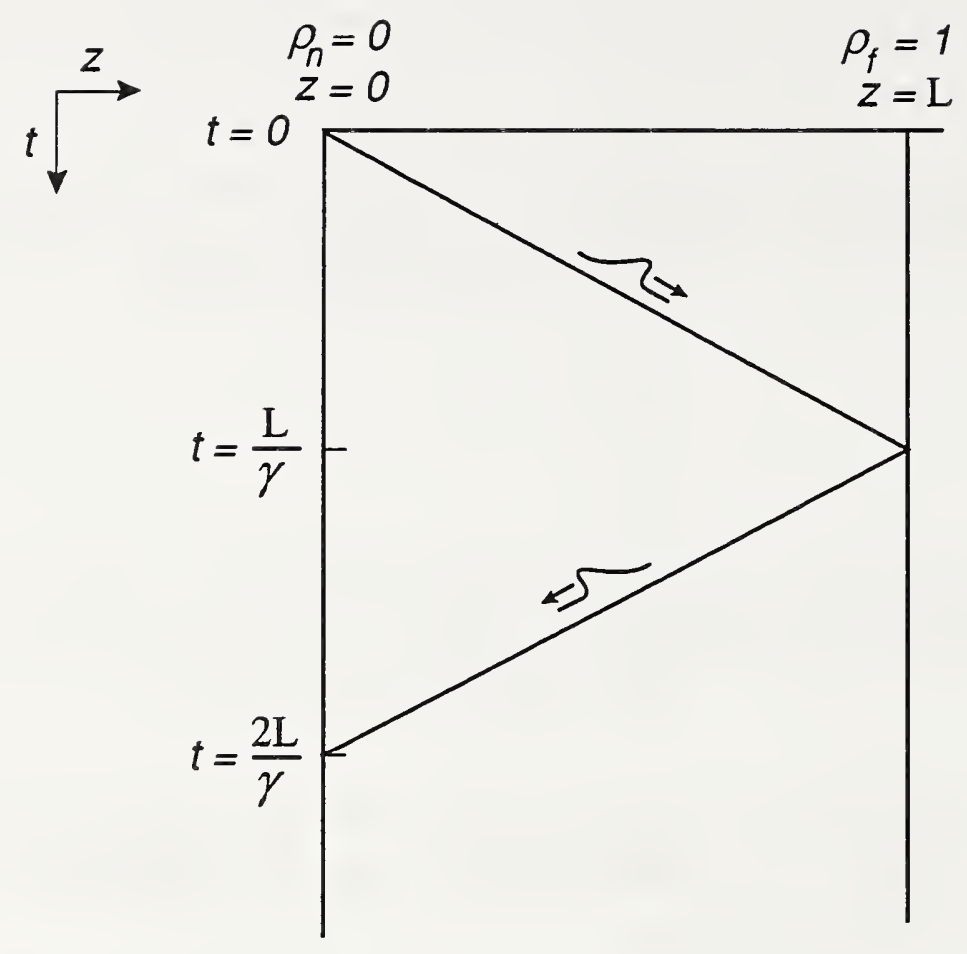

P-D $6-5$

Figure 6-5. Lattice diagram for propagation of $\mathrm{PD}$ pulses

The case of most interest is voltage, $V_{n}$, at the near end $z=0$ when the reflection coefficients are $\rho_{n}=0$ and $\rho_{f}=1$. This case corresponds to an instrument measuring the voltage at the position $z=0$ due to a partial discharge located at $z=x_{i}$ on a cable terminated in its characteristic impedance at $z=0$ and left open-circuited at the other end, $z=\mathrm{L}$. This same solution also applies to the first two terms of the expansion when the instrument end also has a reflection coefficient of one. The solution is given as

$$
V_{n}(0, \omega)=\frac{I(\omega) Z_{0}}{2}\left[e^{-\gamma(\omega)\left(2 \mathrm{~L}-x_{i}\right)}\right]
$$

where the characteristic impedance is considered to be real valued. The first part in the brackets is referred to as the direct contribution and the second part in the brackets is referred to as the reflected contribution.

There are approximate methods that lend understanding to the solution for the first few reflections. One simple tool that aids in the understanding of pulse propagation in a cable is the lattice diagram, see figure 6-5. The horizontal axis of the lattice diagram is the distance along the cable and the vertical axis is time. If a pulse is transmitted down the cable it travels with a velocity, $\gamma$, determined by the phase constant, arriving at different positions along the cable as time progresses. The propagation of the pulse is shown on the lattice diagram as a diagonal line whose slope is determined by the propagation velocity. Each point along the diagonal line corresponds to the pulse 


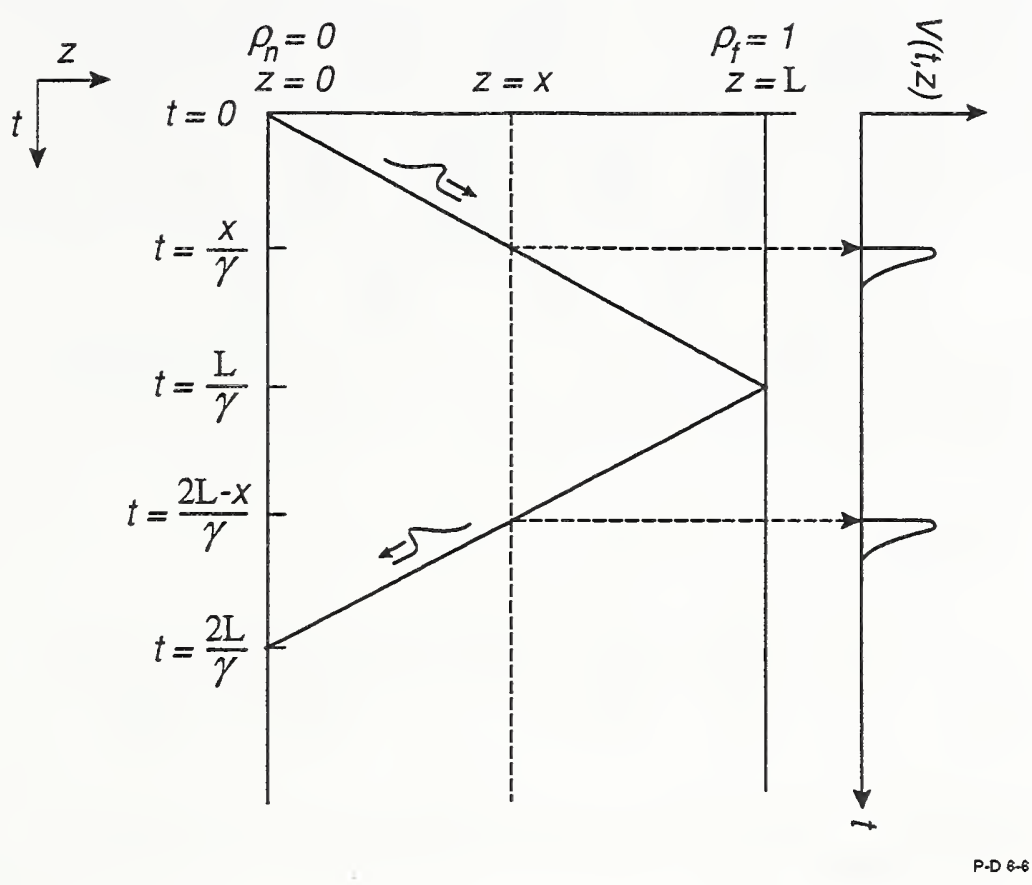

Figure 6-6. Lattice diagram and volt-time plot of PD pulses at point within cable

as it arrives at a particular position in the cable at a particular time. As the pulse arrives at an end of the cable, it is reflected back with an amplitude determined by the reflection coefficient. In this manner it is possible to draw a sketch of the pulse as it travels along the cable.

Consider a simple case in which an instrument measures the voltage on a cable. The instrumentation end is at the left hand side and is labeled as the position $z=0$. The other end of the cable, at the right hand side, is open circuited and is labeled $z=\mathrm{L}$. The cable has a characteristic impedance $Z_{0}^{\prime}$. The instrumentation end is assumed to be terminated in the characteristic impedance of the cable. Since the instrumentation end has a matched impedance the reflection coefficient, $\rho_{n}$, is zero so no signals will reflect from this position. The other end of the cable is an open circuit, making the reflection coefficient, $\rho_{f}$, equal to one. Any signal arriving at this position is reflected back along the cable. The reflection coefficients are labeled on the lattice diagram at their respective ends. Consider a pulse that is injected into the cable at the position $z=0$. As the pulse arrives at the open circuit it is reflected back toward the instrument end. As it returns to the instrumentation end there it is absorbed since the reflection coefficient is zero.

Using the diagram of figure $6-5$ it is possible to sketch rapidly the signal as if one were able to insert a small probe into the cable at a particular position and record the signal as a function of time. This sketching is accomplished by turning the lattice diagram on its side and drawing a second time axis parallel to the time axis of the lattice diagram as shown in figure 6-6. To determine the signal, a line is drawn 


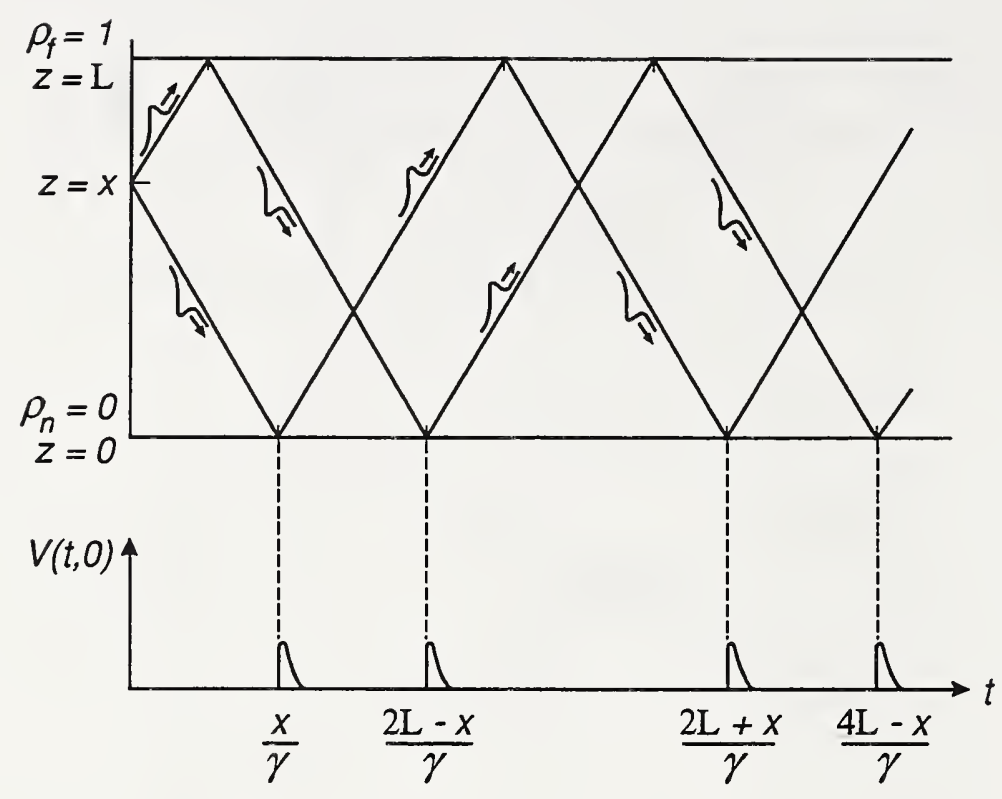

Figure 6-7. Lattice diagram and volt-time plot of PD pulses at near end

parallel to the time axis at the desired probing location, $x$, along the cable. Each time this line intersects the diagonal lines of the lattice diagram a mark is placed on the new time axis. Each mark on the new time axis corresponds to the arrival of a pulse. The pulses are sketched in at these marks, being careful to maintain the polarity determined by the reflection coefficient.

A second example is shown in figure 6-7 and corresponds to the analytical example calculated previously. In this figure the voltage is measured at the position $z=0$, instead of $x$. The cable is terminated in its characteristic impedance at the position $z=0$ and is left open-circuited at $z=\mathrm{L}$. A current source excites the cable at the position $z=x$. Pulses travel in both directions along the cable and correspond to the direct arriving pulse and the reflected pulse. The probing position in this case is $z=0$.

\subsection{Estimation of charge}

An estimate of the charge associated with a PD pulse is necessary to decide whether the PD site is innocuous or is a defect of interest. The noise in the system and the attenuation in the cable limit the instruments ability to estimate accurately the charge. In the absence of a reliable in-situ calibration method one can only hope to make an estimate of PD charge levels from measurement of pulse amplitudes. In general, such estimates are nontrivial and often highly uncertain. The following discussion describes the estimation of the charge along with the theoretical bounds on the estimated quantity. 
The primary cause of signal distortion in cables is the frequency dependent loss. The charge is related to the discharge current as given by Eq. 5-3. However, the measured current pulse is generally significantly distorted at the point of observation. At high frequencies this loss becomes severe and for longer cables, most of the signal energy above $10 \mathrm{MHz}$ is lost. This high attenuation of the signals and the presence of noise imposes a limit on the sensitivity of PD measurements in cables.

There are some fundamental limitations to the measurement of partial discharge which can be stated in terms of a relationship between the bandwidth, the noise, and the attainable sensitivity (Boggs \& Stone, 1982). These results can also be stated in terms of a fundamental lower bound on the estimation of the charge. The bound is referred to as the Cramer-Rao lower bound and is expressed in terms of the minimum variance of any estimator used to estimate the charge in a PD pulse. Whenerer a measurement is made, noise limits its ultimate sensitivity and the Cramer-Rao lower bound defines this ultimate limit in terms of the variance of the noise corrupting the estimate. The variance given by the Cramer-Rao lower bound can be realized by an optimal estimator, however, it is usually diffcult, if not impossible, to determine the optimal estimator.

To calculate the Cramer-Rao lower bound it is necessary to make assumptions about the statistical nature of the noise corrupting the measurement. If the noise is from a stationary, Gaussian random process with power spectral density, $N(\omega)$, then the bound on the variance of the estimate of the amplitude, $\hat{a}$, is giren by (Van Trees, 1968):

$$
\operatorname{var}(\hat{a}-a) \geq\left[\frac{1}{2 \pi} \int_{-\infty}^{\infty} \frac{\left|S_{s}(\omega)\right|^{2}}{N(\omega)} d \omega\right]^{-1}
$$

where $a$ is the unknown, nonrandom amplitude factor applied to the unit amplitude PD pulse $s_{s}(t)$. The amplitude is considered nonrandom in this discussion to simplify the argument and can be extended to the random amplitude case if desired. The amplitude is the appropriate parameter to estimate because an estimate of the charge, on the basis of a unit charge pulse, reduces to an estimate of a scale factor (amplitude). The Gaussian noise assumption is appropriate since the predominant types of noise corrupting the measurement are the thermal noise in the system and AM band radio broadcasts. Furthermore, the Gaussian assumption makes the calculation of the lower bound tractable.

The actual quantity of interest is the charge and these results can be stated in terms of a lower bound on the estimation of the charge (Steiner, 1988). Following (Boggs and Stone 1982), the PD pulse will be assumed to have a Gaussian pulse shape

$$
s_{s}(t)=a \exp \left(-t^{2} / 2 \tau_{0}^{2}\right)
$$


where $a$ is the amplitude to be estimated and $\tau_{0}$ is a constant related to the rms duration of the pulse, the latter defined as

$$
\tau_{r m s}=\sqrt{\frac{\tau_{0}^{2}}{2}}
$$

The Gaussian assumption for the pulse shape should not be confused with any statistical assumption about the partial discharge, it is merely a convenient mathematical tool for expressing the results in a closed form.

For purposes of analysis and comparison with the charge, a dc-coupled system is assumed since charge cannot be directly measured unless the dc component is present. The charge in this calculation is assumed to be the actual charge and not an apparent charge. While this is unrealistic from a practical standpoint, discussing the lower bound in terms of an apparent charge unnecessarily complicates the discussion. Since the relationship between the charge and the apparent charge is a scale factor, the results are still valid. Inclusion of this scale factor simply reduces the sensitivity by the same amount. Also, so that the result can be easily expressed in closed form, the noise will be assumed to be white Gaussian noise. The charge, $q$, in the pulse can be calculated to be

$$
q=\frac{a}{2 Z_{0}} \sqrt{2 \pi \tau_{0}^{2}}
$$

where $Z_{0}$ is the characteristic impedance of the cable.

The quality of the measurement is generally defined in terms of a minimum acceptable signal-to-noise ratio, SNR. The output signal-to-noise ratio, $\mathrm{SNR}_{\text {out }}$, of the estimator is given by

$$
\mathrm{SNR}_{\text {out }}=\frac{a^{2} E_{\mathrm{s}}}{N_{0} / 2}
$$

where $E_{s}$ is the energy of the unit amplitude pulse. The noise, $N_{0}$, is defined as $k \mathrm{~T}_{\text {eq }}$ where $k$ is Boltzmann's constant and $\mathrm{T}_{\text {eq }}$ is the equivalent noise temperature of the entire system. The effective noise temperature includes contributions to the thermal noise from the cable, measurement impedance and amplifier. For practical purposes, a subjectively chosen value is assigned to $\mathrm{SNR}_{\text {out }}$. Using these assumptions, the minimum measurable charge is given by

$$
Q_{\min }=\left(\frac{8}{\pi}\right)^{\frac{1}{4}} \frac{1}{Z_{0}}\left[\frac{\mathrm{SNR} \text { out } N_{0} / 2}{B}\right]^{\frac{1}{2}}
$$


where $B$ is the rms bandwidth of the PD pulse and is given by

$$
B=\frac{1}{\sqrt{8 \pi^{2} \tau_{0}^{2}}}
$$

This calculation assumes a matched impedance load on the cable so that maximum energy is transferred from the cable to the detector. Most commercially available PD detectors have circuits that reduce the available signal level. This loss of signal energy can also be described by a scale factor which, when included, further reduces the sensitivity.

As an example of the application of Eq. 6-36, consider a $35 \Omega$ cable having a length which limits the pulse bandwidth to $5 \mathrm{MHz}$. Furthermore, assume that the system has a noise figure of 4 so that $N_{0}$ is $32 \times 10^{21}$ and that a measurement with a $20 \mathrm{~dB}$ SNR is the minimum acceptable result. Using these values the minimum charge that can be measured is $0.014 \mathrm{pC}$. This particular result represents the theoretical minimum charge that can be measured if the optimum estimator is used in a $35 \Omega$, matched impedance system.

The result in Eq. 6-36 should only be viewed as an approximate result since it assumes white noise and a Gaussian pulse shape. Using Eq. 6-32 it is possible to calculate numerically more precise lower bounds if accurate models (or estimates) are used for the pulse shape and noise.

Even if Gaussian noise is assumed, it is still difficult to find an optimal estimator for partial discharge. The difficulties with determining an optimal estimator are related to the available information about the received signal. To determine the optimum estimator it is necessary to know the exact propagation characteristics of the cable, the length of cable through which the PD pulses have traveled, the exact arrival time of the PD pulse, the statistical distribution of the charge, and the statistical characteristics of the Gaussian noise. None of this information is available and either has to be estimated or assumed. Imprecise information about these quantities will cause additional loss in performance; however, this loss will be moderate since estimators can be found which are nearly optimum.

One possible estimator that is nearly optimum, is the linear minimum mean square error (LMMSE) estimator of the amplitude (Franks, 1981). This particular estimator is based on a linear filter which is optimum in the class of all linear estimators. The filter, $H_{\text {opt }}$, is given by

$$
H_{\text {opt }}(\omega)=\frac{\alpha S_{s}^{*}(\omega) \exp \left(-j \omega t_{i}\right)}{N(\omega)}
$$


where $N(\omega)$ is the power spectral density of the noise described in Section $6.1, t_{i}$ is the arrival time of the ith pulse with amplitude $a_{i}$ and the superscript, ${ }^{*}$, means the complex conjugate. The scale factor $\alpha$ is given by

$$
\alpha=\frac{\overline{a^{2}}}{1+\overline{a^{2}} \int_{-\infty}^{\infty}\left|S_{\mathrm{s}}(\omega)\right|^{2} / N(\omega) d \omega}
$$

where $\overline{a^{2}}$ is the mean square value of the random variable, $a_{i}$, (the amplitude of the PD pulse) that is being estimated and $S_{\mathrm{s}}(\omega)$ is, in essence, the Fourier transform of the propagation characteristic of the cable. The estimate of $a_{i}$ is then determined as

$$
\hat{a}_{i}=\left.\mathcal{F}^{-1}\left\{X_{r}(\omega) H_{o p t}(\omega)\right\}\right|_{t=0}
$$

where $\mathcal{F}^{-1}$ refers to the inverse Fourier transform and $X_{r}(\omega)$ is the received PD pulse corrupted by additive noise.

If the noise is Gaussian, then the LMMSE estimator is the optimum estimator; however, even if the noise is not Gaussian, this estimator is the optimum linear estimator.

The variance of the estimate is not the only measure of error that is important. Bias errors in the measurement can also be important and are defined as

$$
\operatorname{Bias}[\hat{a}]=E\{a-\hat{a}\}
$$

where $E$ is the expectation operator, $a$ is the actual value of the quantity being estimated and $\hat{a}$ is its estimate. In this case, the bias error can take the form of an unwanted scale factor. For example, an incorrect choice of the pulse shape (cable characteristics) can cause a scaling error in the estimate, implying that the appropriate $S_{\mathrm{s}}(\omega)$ needs to be used in the filter described by Eq. 6-39. This implication requires that the filter in Eq. 6-39 be adaptive in the sense that $S_{\mathrm{s}}(\omega)$ must correspond to the particular length of cable through which the PD pulse traveled.

The LMMSE is a near, optimal estimator of the charge but requires a complicated and numerically intensive computation. A simpler method that yields good results can be used. For this method, an ad hoc autocorrelation estimator is used. It is based upon a maximum likelihood argument used in a different context and it should be noted that this estimator is not a maximum likelihood estimator for this case. The estimate is formed by evaluating the autocorrelation function at zero delay, removing the bias due to the noise, and taking the square root of the result. The value, $y$, of the time autocorrelation function at zero delay is defined by 


$$
y=\int_{-T / 2}^{T / 2} x_{r}^{2}(t) d t
$$

where the $\mathrm{PD}$ pulse is assumed to be contained in the interval $T$ and $x_{r}$ is the received PD signal and noise. In the usual definition of the time autocorrelation function, the result is normalized by the factor $1 / \mathrm{T}$. In Eq. 6-42, this factor has been omitted for convenience. The resulting value will be an estimate of the sum of the noise energy and the energy in the partial discharge pulse. Taking the expected value yields

$$
\mathcal{E}\left\{y \mid a_{i}\right\}=a_{i}^{2} \mathrm{e}_{s}+T \sigma^{2}
$$

where $\sigma^{2}$ is the variance of the noise and $e_{s}$ is the energy in the signal pulse, $s(t)$. The term $T \sigma^{2}$, is an unwanted bias term and is removed from the calculation before further processing. The final step is to calculate the square root of the result after removal of the bias. The estimate of the amplitude is then

$$
\hat{a}_{i}=\frac{\sqrt{y-T \sigma^{2}}}{a_{\text {calib }}}
$$

where $a_{\text {calib }}$ is the reference level obtained through calibration first performed with no PD occurring, then with a known PD pulse amplitude and $T \sigma^{2}$ is also obtained through calibration.

One interesting sidelight is that the autocorrelation method can provide meaningful results when the $\mathrm{PD}$ pulse spacing is too small to resolve individual pulses. Consider the case in which the observed signal is

$$
x_{r}(t)=\sum_{i=1}^{N_{T}} a_{i} s_{s}\left(t-t_{i}\right)+n(t)
$$

where $t_{i}$ is a time of arrival of the ith pulse and where there are now $N_{T}$ pulses in the interval $(-T / 2, T / 2)$. If the process is a homogeneous Poisson process with intensity parameter $\lambda$, then the estimate of the autocorrelation function at zero delay becomes

$$
y=\lambda \overline{a^{2}} s_{s}+T \sigma^{2}
$$

where $\overline{a^{2}}$ is the mean square value of the amplitude. The output of the estimator is slightly different because it now gives an estimate of the square root of $\lambda \overline{a^{2}}$. We no longer get an estimate of the magnitude of the charge but an estimate of its average rms value.

The set of measurements in figure 6-8 illustrate the relative performance of three 


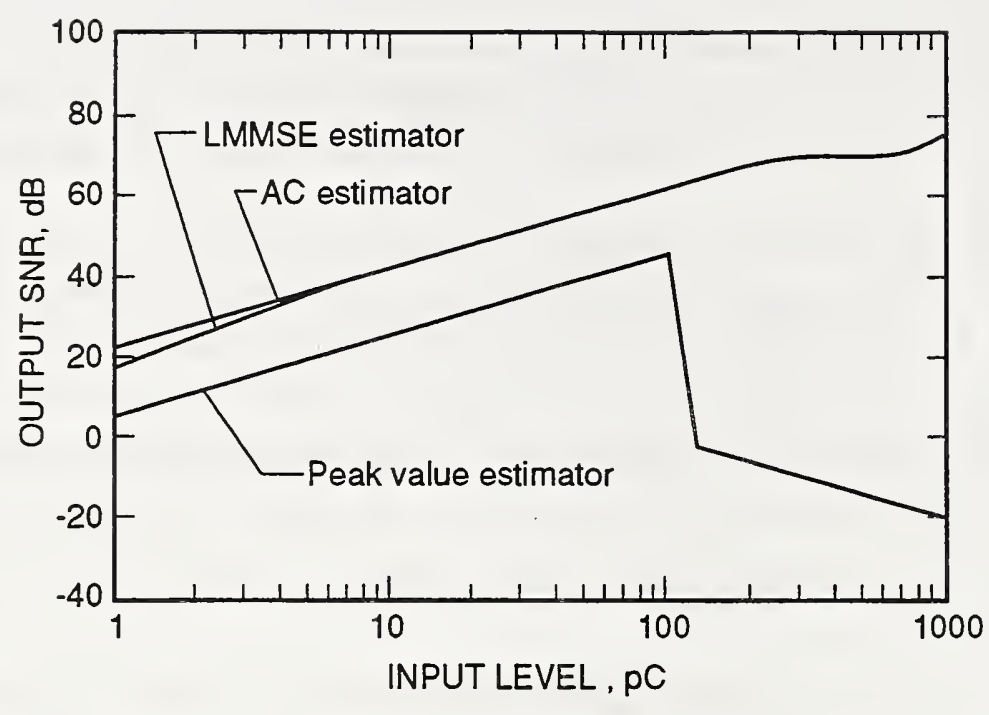

Figure 6-8. Relative performance of estimators

different estimators obtained using an 8-bit quantizer. The measure of performance is the signal-to-noise ratio defined as

$$
\mathrm{SNR}=10 \log \left[\frac{\text { mean of estimate squared }}{\text { mean square of estimate }}\right] .
$$

In these measurements the rms error is due only to the variance of the estimator because the digitizer was operated in its linear region. The input signal was maintained at a fixed level of about $10 \%$ of the full scale input level of the digitizer. The input noise level was adjustable so that the input SNR could be varied between $10 \mathrm{~dB}$ and $30 \mathrm{~dB}$. illustrates the effectiveness of both the LMMSE and autocorrelation estimators in noisy measurements while the peak value estimator tracks the input SNR. The autocorrelation estimate performed better than the LMMSE estimate at low SNR's because the pulse arrival time also had to be estimated.

As illustrated by the measurements, the LMMSE and autocorrelation estimators outperform the simple peak-value estimator. Figure 6-8 shows that the peak value estimator has a sudden loss of performance when the peak of the waveform is clipped by the digitizer (at $100 \mathrm{pC}$ ). Even when the waveform is severely clipped both the LMMSE and the autocorrelation estimators provide useful results. However, the LMMSE technique requires relatively sophisticated computations that are time consuming. Special purpose hardware is available to accelerate the calculations, but is expensive. The autocorrelation method provides estimates that, in these measurements, were as good as the LMMSE estimate. The major advantage of the autocorrelation estimator is that it is much simpler to implement, requiring only integration and simple scalar operations. 


\subsection{Partial discharge location}

When voltage is applied to the measurement system, partial discharges occurs at various positions within the system but only partial discharges within the interior of the cable are of interest. The ability to calculate the position of the partial discharges within the system permits the computer to discriminate between partial discharges occurring interior to the cable and exterior to the cable. Furthermore, with the capability of calculating the position where each individual pulse originates, it is also possible to determine the statistical characteristics of the partial discharges from individual sites.

The signal parameters of interest are the time of occurrence of the PD pulse and the position within the cable from which it emanates. The parameter estimates that yield these quantities are called delay estimates and are estimates of the times of arrival of the pulses. From a fundamental point of view, there is essentially no difference in the methods used to determine the time of occurrence or the location of the discharge. Both of these quantities are derived from the same estimate. It is simply a matter of how the delay estimates are scaled after they are determined.

Some alternative methods for estimating the $\mathrm{PD}$ location are discussed in the following along with their strengths and weaknesses. In each case one end of the cable is instrumented, and the other end is open.

To understand the estimation procedure, it is informative to consider a simple case of lossless, nondispersive propagation with a matched impedance at the receiving, near end of the cable. The matched impedance termination at the receiver is not necessary if only the first two arriving pulses are used, however, to simplify the explanation a matched termination is assumed. In this case the cable is a linear phase system with the propagation phase function given by

$$
\beta(\omega)=\frac{1}{\gamma} \omega=\frac{1}{\sqrt{L C}} \omega
$$

where $\gamma$ is the propagation velocity and is assumed to be a constant that depends on the inductance and capacitance per unit length of the cable as defined in Section 6.2. The noiseless, received signal, $S_{\mathrm{s}}(\omega)$, is described in the frequency domain as

$$
S_{\mathrm{s}}(\omega)=I_{s}(\omega) H_{c}(\omega)
$$

where $I_{s}(\omega)$ is the Fourier transform of a single PD current pulse generated at the defect site. For simplicity, the linear filter, $H_{c}(\omega)$, describes the propagation through a loss-less and nondispersive cable and is given by 


$$
H_{c}(\omega)=e^{-j \omega \frac{1}{\gamma} x_{i}}+e^{-j \omega \frac{1}{\gamma}\left(2 \mathrm{~L}-x_{i}\right)}
$$

where $L$ is the total length of the cable and $x_{i}$ is the length of cable between the receiver near end and the $\mathrm{PD}$ site. This equation is a special case of the solution given in Eq. 6-27 and has a simple explanation. Each of the exponential terms corresponds to a time delayed impulse with the time delay corresponding to the propagation delay from the PD site to the receiver. This is easily seen by calculating the inverse Fourier transform of $H_{c}(\omega)$ which is

$$
\mathcal{F}^{-1}\left\{H_{1}(\omega)\right\}=\delta\left(t-\frac{x_{i}}{\gamma}\right)+\delta\left(t-\frac{2 \mathrm{~L}-x_{i}}{\gamma}\right)
$$

If the digitizer begins recording at the instant that the partial discharge pulse occurs, then the first impulse (delta function) is the direct arriving pulse and arrives at time $\mathrm{t}_{1}$ given by

$$
t_{1}=\frac{x_{i}}{\gamma}
$$

The second impulse is the reflected term that arrives at time $t_{2}$ given by

$$
t_{2}=\frac{2 \mathrm{~L}-x_{i}}{\gamma}
$$

The location of the PD site is deduced from an estimate of the time difference, $\Delta t_{a}$, between the direct pulse and the reflected pulse and is given by

$$
\Delta t_{a}=t_{2}-\frac{2\left(\mathrm{~L}-x_{i}\right)}{\gamma}
$$

The position of the $\mathrm{PD}$ site is then calculated as

$$
\frac{1}{2} \gamma \Delta t_{a}=\mathrm{L}-x_{i}
$$

Here, the calculated position within the cable is the distance from the far end of the cable to the PD site.

It is interesting to consider what a priori information about the cable propagation characteristics is actually required. This testing application is unusual because one is not necessarily interested in the exact position of the defects within the cable, but only their presence. The only required pieces of information about the pulse 
are: (1) that it originated from the interior of the cable and (2) its relative position within the cable. Referencing the location from the open circuit end of the cable removes the requirement of knowing the total length of the cable. After performing a calibration experiment to determine the electrical length of the cable one can then choose an arbitrary physical length for the cable, for example one meter, to calculate the velocity. The measurements in effect become relative positions within the cable. The result is that no a priori information about the physical dimensions of the cable are required for these measurements.

The simplest method for determining the time delay is to use the time at which the received signal crosses some predetermined amplitude threshold. The threshold is chosen to be some small level near the zero baseline of the signal. When the direct-arriving pulse crosses the threshold a timer is started, and is stopped when the reflected pulse crosses the same threshold. This method has the adrantage that results can be accumulated in real time without the need for time-consuming intervention by a computer to perform calculations.

Basically this is the same approach used in time domain-reflectometry (TDR) when the operator views the pulses on the TDR screen and measures the time delays between pulses. The difference is that in this approach, the measurement is automated and that the pulses are generated by the PD process rather than by the instrument. The method will be referred to as the TDR method and has limited application because of the difficulty in determining the appropriate threshold for distorted, noisy, or unknown waveforms. If the signal is distorted with excessive ringing the oscillations will cause false triggering of the timing circuits. If noise is present it can cause false triggering of the timer, especially in the case when low lerel partial discharges are measured. If two pulses are overlapping or closely spaced, the timer will begin counting but will not have a trigger to stop. The cable is dispersive and consequently neither the front edge of the pulse nor the pulse shape is preserved. In the typical cable, attenuation is heavy so the reflected pulse is often much less in amplitude than the direct pulse. These nonideal propagation characteristics contribute to the difficulty of obtaining a delay estimate using a predetermined amplitude threshold as a trigger reference for the two pulses. Partial discharges of both polarities occur and the detection impedance is high-pass in nature so each received pulse has components of both polarities regardless of the initial polarity. This further compounds the difficulties associated with implementing circuitry to properly respond to arbitrary polarities. Any of these problems can cause large errors in the location estimate.

In general, the peak of the pulse waveforms is also an unsuitable reference point on the waveform because of the shifts in the peaks resulting from nonideal propagation characteristics. The basic difficulty associated with the TDR method is the lack of a reference point on the pulse waveform that can be used as a time-delay measurement reference. 
Another method for locating partial discharges in the cable is to calculate the autocorrelation function of the received waveforms. The time autocorrelation is defined by

$$
\mathcal{R}(\theta)=\frac{1}{T} \int_{-\frac{T}{2}}^{\frac{T}{2}} s(t) s(t+\theta) d t
$$

where $s(t)$ is the PD signal defined for Eq. 6-6, $\theta$ is the correlation function argument, and $T$ is the measurement observation time. In the frequency domain the time autocorrelation function is called the energy spectral density and it is given by

$$
|S(\omega)|^{2}=\left|I(\omega) H_{c}(\omega)\right|^{2}=|I(\omega)|^{2}\left[1+e^{-j \omega \frac{1}{\gamma} 2\left(\mathrm{~L}-x_{i}\right)}+e^{j \omega \frac{1}{\gamma} 2\left(\mathrm{~L}-x_{i}\right)}\right]
$$

To interpret this result it is informative to consider only the terms within the brackets of Eq. 6-57. Calculating inverse Fourier transform of the terms in the brackets gives

$$
\mathcal{F}^{-1}\left\{|S(\omega)|^{2}\right\} \approx \delta(\theta)+\delta\left(\theta-\frac{2\left(\mathrm{~L}-x_{i}\right)}{\gamma}\right)+\delta\left(\theta+\frac{2\left(\mathrm{~L}-x_{i}\right)}{\gamma}\right)
$$

This equation has an impulse function at the time origin and at time delays symmetric about the origin. Autocorrelation functions always have their maximum at the origin so the impulse at the origin conveys no information. In this method the location of the PD site $(i)$ is deduced from an estimate of the time delay, $\theta_{i}$, from the origin to either of the impulses symmetrically located about the origin

$$
\left|\theta_{i}\right|=\frac{2\left(\mathrm{~L}-x_{i}\right)}{\gamma}
$$

The position of the PD site is then calculated as

$$
\frac{1}{2} \gamma\left|\theta_{i}\right|=\mathrm{L}-x_{i}
$$

Here again, the calculated position within the cable is the distance from the far end of the cable to the PD site, noting that the result is the same as that of Eq. 6-55 but using a correlation function.

For best performance, it is necessary that the near end of the cable be terminated in its characteristic impedance. There will always be unwanted reflections from the receiver because it is difficult to terminate a cable in a matched impedance when high voltage is present. However, it is possible to make the reflection coefficient at the receiver small, at least across a limited band of frequencies. Due to the impedance mismatch at both ends of the cable, the autocorrelation function will be a superposition of the direct 
term, the first reflected term, and of all the secondary reflections. The delay term of interest will have contributions from all these reflections. The secondary reflected terms in the superposition are highly dispersed and have phase shifts incurred during reflection from the receiver. These secondary reflections cause the desired delay term to accumulate errors from the dispersion and phase shifts.

The received signal used in the calculation of the autocorrelation function has noise and the autocorrelation function of this noise is added to the desired autocorrelation function. If the noise is wideband thermal noise, the added term is concentrated around the origin $\left(z_{i}=0\right)$ and is of little consequence. Usually the noise is from narrowband external interference entering at the cable terminations and contributes a term for the delay corresponding to the cable ends. The contribution from the noise correlation function can obscure PD sites near both ends of the cable and, if strong enough, can obscure sites located far within the interior of the cable.

When partial discharges are located near either end of a long power cable, the pulse resolution of the measurement may not be great enough to locate the PD site. Since the measurement is made from only one cable end, the method relies on the directlypropagated PD pulse and its reflection from the open-circuit end of the cable. If the partial discharge site is located close to the far end of the cable, the arrival times of the direct pulse and reflected pulse are almost the same, and it becomes difficult to differentiate between a single pulse and two closely spaced pulses. The two pulses will be interpreted as one and attributed to a partial discharge at the cable termination. When this occurs, the measuring system is said to be resolution-limited since it cannot resolve both pulses. The region of the cable in which the direct pulse and reflected pulse are not resolved is called the blind spot of the cable. This type of measurement resolution limitation can be described in terms of a delay bias error (Steiner, 1988) and will be called Type I resolution bias in this report. As a rule of thumb, the two pulses are resolvable if the PD site is located a distance from the far end of the cable corresponding to a propagation delay (equivalent distance) equal to the reciprocal of the bandwidth of the received PD signals. Typical bandwidths for practical lengths of cable are no more than $10 \mathrm{MHz}$ (100 ns reciprocal), so any $\mathrm{PD}$ sites within $15 \mathrm{~m}$ (assuming a propagation delay of $6.56 \mathrm{~ns} / \mathrm{m}$ ) from the far end of the cable will appear as a partial discharge at the far end of the cable. Long cables have even narrower bandwidths, further reducing the resolution.

\subsubsection{Matched filter}

An improvement in the autocorrelation technique is possible using matched filtering (Steiner, 1988). This technique cross-correlates a calibration waveform with the received signal. Mathematically this operation is defined by 


$$
m(t)=\int_{-\infty}^{\infty} a_{i} s_{s}(\theta) h_{m}(\theta-t) d \theta
$$

where $s_{s}(\theta)$ is the received PD signal, previously defined and $h_{m}(t)$ is a time-reversed version of a calibration waveform similar to the $P D$ signals but including only the direct propagation term

$$
H_{m}(\omega)=S_{\text {direct }}^{*}(\omega)
$$

where $S_{\text {direct }}^{*}(\omega)$ is the directly arriving component of the time-reversed PD pulse. In the frequency domain, the matched filtering operation is described by

$$
M(\omega)=\frac{a_{i} I_{s}(\omega) Z_{0}}{2} H_{m}(\omega)\left[e^{-j \omega \frac{1}{\gamma} x_{i}}+e^{-j \omega \frac{1}{\gamma}\left(2 \mathrm{~L}-x_{i}\right)}\right]
$$

This result is interpreted by calculating the inverse Fourier transform of the terms within the brackets giving

$$
\mathrm{F}^{-1}\{M(\omega)\} \approx \delta\left(t-\frac{x_{i}}{\gamma}\right)+\delta\left(t-\frac{\left(2 \mathrm{~L}-x_{i}\right)}{\gamma}\right)
$$

This result is the same result found with autocorrelation, so the partial discharge site is located by interpreting the time delays in the same manner. As before, the calculated position of the PD site is the distance from the far end of the cable to the PD site.

In this simple analysis, the term $I_{s}(\omega) H_{m}(\omega)$ was ignored because, by supposition, the filter $H_{m}(\omega)$ is matched to the shape $I(\omega)$ and does not contribute to the delay (in the absence of dispersion). The term $I_{s}(\omega) H_{m}(\omega)$ is in essence the autocorrelation function of the directly-arriving PD pulse. In fact, the inverse Fourier transform of $M(\omega)$ is the superposition of time-shifted autocorrelation functions

$$
m(t)=R_{\text {direct }}\left(t-\frac{x_{i}}{\nu}\right)+R_{\text {direct }}\left(t-\frac{2 L-x_{i}}{\nu}\right)
$$

where $R_{\text {direct }}(t)$ is the autocorrelation function of the directly-arriving PD pulse. The advantage of this technique is related to the interpretatiom of the autocorrelation function. By definition, the autocorrelation function has its maximum at the time origin. This means that the positions of peaks of the time-shifted version correspond to the correct time delays. The inability to choose a consistent position on a waveform as the time reference causes the level-crossing technique to be highly inaccurate. In contrast, the autocorrelation method has an inherent time reference, the peaks of the autocorrelation function. In the matched filter method, the times of arrival are the 
times at which the output correlation function attains its maxima. This approach yields excellent time-delay estimates, provided that the signal shape is accurately known beforehand, because the problems associated with selecting a proper threshold are eliminated. This technique also performs adequately in the presence of noise.

A calibration experiment can provide an adequate waveform for use as the matched filter. The received signals travel through unknown amounts of cable so the shape will be slightly different than the calibrating signal. This inaccuracy in pulse shape causes a bias error in the delay estimate because of dispersion. However, the major problem with this method is due to multiple pulses arriving at almost the same time. The matched filter provides only slightly better results than the level-crossing method, and the same performance as the autocorrelation method.

In the presence of external interference, the matched filter provides slightly better results than the autocorrelation method. The matched filter can suppress noise outside its bandwidth, thus improring performance. Also, the autocorrelation method concentrates the noise at a single delay; obscuring sites near this delay; the matched filter does not.

\subsubsection{Multiple sites}

Correlation-based methods can use averaging to reduce the effects of noise. However, when averaging is used, another tvpe of resolution error occurs. The correlation function has its peak centered at the time delay corresponding to the PD site. Correlation functions are similar in width to the partial discharge pulses used in their calculation. Resolution problems occur if two PD sites are located in close proximity to each other. In this case, the correlation functions overlap and it is not possible to distinguish two indiridual correlation functions; they appear as one. This case is another distinct type of resolution limitation of the measurement and will be called Type II resolution bias in this report. As a rule of thumb, the two sites are resolvable if the sites are separated by a propagation delay (equivalent distance) that is the reciprocal of the bandwidth of the received PD signals. Typical cables severely bandlimit the received signals and for practical lengths of cable bandwidths are no more than $10 \mathrm{MHz}$ ( $100 \mathrm{~ns}$ reciprocal). This limitation means that $\mathrm{PD}$ sites must be separated by $15 \mathrm{~m}$ (assuming a propagation delay of $6.56 \mathrm{~ns} / \mathrm{m}$ ), and only $33 \mathrm{PD}$ sites can be resolved in a $500 \mathrm{~m}$ length of cable. The resolution performance decreases as the cable length increases.

Fortunately, there is a simple method that greatly reduces the effects of this resolution limit. If the location of the partial discharge site is calculated from each data record and sorted into a histogram, then the resolution can be increased substantially. Ideally, the histogram consists of single impulses, each corresponding to a separate PD site. However, the estimates of the time delays are noisy and this noise is described by the variance of the estimates. When the histogram is formed, the variance of 


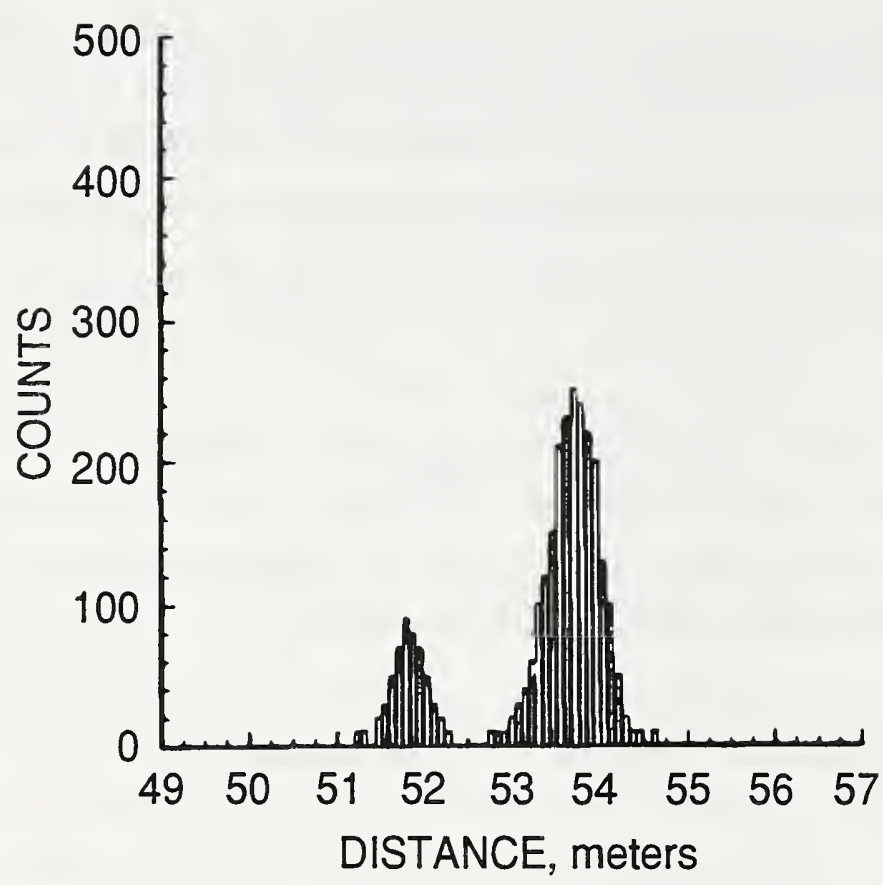

P-D 6-9

Figure 6-9. Delay histogram for two PD sites located $2 \mathrm{~m}$ apart in a $90 \mathrm{~m}$ cable.

the estimate is a measure of the width of the histogram of the estimates. Better delay estimates (smaller variance) result in narrower histograms and thus better resolution. The estimator with the smallest variance should be used to increase the resolution. Resolution is not limited to increments of the sampling frequency; it is possible to interpolate between points to within fractions of the sampling rate. As an example, consider a cross-correlation estimate of a $0.025 \mathrm{pC}$ partial discharge with a $10 \mathrm{MHz}$ bandwidth and a $14 \mathrm{~dB}$ SNR. It can be shown that the standard deviation of this estimate is $7 \mathrm{~ns}$ (Steiner, 1988). Stronger PD signals have smaller variances, further increasing the resolution. The resolution for this example is $1 \mathrm{~m}$, a 15 -fold increase; 500 sites could be located in a $500 \mathrm{~m}$ cable, but multiple sites within any $1 \mathrm{~m}$ length will appear as a single site. This approach was used to detect the presence of two closely spaced PD sites in a $90 \mathrm{~m}$ cable. Both partial discharge sites are easily identified using the delay histogram in figure 6-9 even though they are only separated by $2 \mathrm{~m}$. To achieve this same resolution with a correlation function measured using signal averaging would require a signal bandwidth in excess of $70 \mathrm{MHz}$, which is impossible with this length of cable.

There is the possibility that erroneous delay values may be calculated from the data. When partial discharges from two different locations are recorded in the same data record, the delay estimate may not correspond to either of the true locations. The calculated delay may be the time between the occurrence of the two partial discharges. It was stated that the probability of this occurrence is small but not negligible. One concern is that these multiple events may cause a build-up in the delay histogram at a particular delay giving a false indication of a PD site. It is presumed that the individual PD sites are statistically independent from one another. The times 
between events at the individual sites are random so the relationship between times of occurrence at different sites will also be random. Measuring the time delays between pulses from different sites will result in an estimate of the conditional probability density of the times between these independent events, conditioned on the fact that the time difference is less than a record length. The contribution from these interoccurrence times to the delay histogram will not result in a peak at any of the delays of interest.

\subsection{Enhancements in the signal analysis}

The experimental work performed during this study was, by charter, an assessment of existing methods. Three possible enhancements might be applied in future work, improving the results. The first would be to generalize the matched filter; the second would be to eliminate the blind zone at the far end of the cable. Third, a crosscorrelation method could be used if indeed the far end of the cable becomes available as a result of the need to disconnect equipment at that end of the cable.

\subsubsection{Generalized matched filter}

There are generalizations to the matched filter approach that can improve the measurement in the presence of external interference (Franks, 1981). If external interference is present, then using a filter similar to that used in the Linear Minimum Mean Square Error (LMMSE) charge estimator gives essentially the same results as the simple matched filter except that the interference is attenuated. The filter is similar to a Wiener filter and is given by

$$
H_{W}(\omega)=\frac{\xi \overline{a^{2}} S_{\mathrm{s}}^{*}(\omega)}{\overline{a^{2}} \psi\left|S_{\mathrm{s}}(\omega)\right|^{2}+N(\omega)}
$$

where $\psi$ is a parameter that controls the pulse resolution of the filter and $N(\omega)$ is the noise previously defined (Franks, 1981). The scale factor $\xi$ is given by

$$
\xi=\left[1+\int_{-\infty}^{\infty} \frac{\overline{a^{2}}|S(\omega)|^{2} d \omega}{\overline{a^{2}} \psi|S(\omega)|^{2}+N(\omega)}\right]^{-1}
$$

'where the term $\overline{a^{2}}$ is the mean square value of the amplitudes (Steiner, 1988). The interpretation of this filter is aided by considering the denominator of Eq. 6-67. At frequencies where the noise power is large, the denominator becomes large so the filter attenuates the noise. The effect is to remove the interference. An additional benefit of this method is that the resulting amplitude of the filtered PD signal is an 
optimum estimate of the charge in the pulse. It is possible to make this filter behave as a deconvolution filter by adjusting the factor $\psi$ to reduce the Type I resolution bias, but this will only reduce the bias by a factor of two to three (Steiner, 1987; Steiner, 1988). The penalty for using deconvolution is an increase in the variance of the delay estimate, and a reduction in the resolution of multiple sites.

\subsubsection{Embedding}

Type II resolution bias is effectively mitigated using delay histograms but Type I resolution bias remains a problem for single-ended measurements. There is a simple solution that corrects this type of error. The solution is to guarantee that all partial discharges are in the region of the cable interior to the blind spots at either end. This solution is accomplished by extending the ends of the cable under test with lengths of $\mathrm{PD}$-free cable that are longer than the extent of the blind spots, in effect embedding the test cable between the two lengths of added cable. The connections from the extensions to the cable under test will cause minor impedance discontinuities but will not have a deleterious influence on the measurement.

\subsubsection{Cross-correlation}

The cross-correlation method uses signals received at both ends of the cable and is effective in eliminating Type I resolution bias in measurements of PD sites located near the ends of a cable (Steiner, 1988). Consider the same simple, lossless, linear phase case as before except now the cable is terminated in matched loads at both ends. The time cross-correlation function is defined as

$$
R_{\text {snsf }}(\theta)=\frac{1}{T} \int_{0}^{T} s_{n}(t) s_{f}(t+\theta) d t
$$

where the signals $s_{n}$ and $s_{f}$ are the signals received respectively at the near end and at the far end of the cable. In the frequency domain, $R_{s n s f}(\theta)$ is called the cross-energy spectral density and it is given by

$$
\mathcal{F}\left\{R_{\text {snsf }}(\theta)\right\}=\frac{\left|\overline{a^{2}} I(\omega) Z_{0}\right|^{2}}{4}\left[e^{-j \omega \frac{1}{\gamma} x_{i}} e^{j \omega \frac{1}{\gamma}\left(\mathrm{L}-x_{i}\right)}\right]
$$

This result is interpreted by calculating the inverse Fourier transform of the term in the brackets giving

$$
R_{\text {snsf }}(\theta) \approx \delta\left(\theta-\frac{\mathrm{L}-2 x_{i}}{\gamma}\right)
$$


In this method the location of the PD site is deduced from an estimate of the time delay, $\theta_{i}$, from the origin of the cross-correlation to the impulse displaced from the origin

$$
\theta_{i}=\frac{\left(L-2 x_{i}\right)}{\gamma}
$$

In the case of cross-correlation, the position of the PD site with respect to the near end is then calculated as

$$
x_{i}=\frac{1}{2}\left[L-\theta_{i} \uparrow\right]
$$

This method does not rely on any reflections and thus does not have a Type I resolution bias, thus the cable has no blind spot.

Cross-correlation provides a significantly higher SNR than single-ended methods. The improvement derives from the fact that single-ended methods rely on a highly attenuated reflection. The improvement is seen by comparing autocorrelation with cross-correlation. In autocorrelation the portion of the correlation function that is used for the delay estimate suffers an attenuation of

$$
\kappa_{a}(\omega)=\epsilon^{-\alpha(\omega)\left[2\left(L-x_{i}\right)\right]}
$$

In cross-correlation the portion of the correlation function that is used for the delay estimate suffers an attenuation of

$$
\kappa_{c}(\omega)=e^{-\alpha(\omega)\left(L-x_{i}\right)} e^{-\alpha(\omega) x_{i}}
$$

Taking the ratio gives the loss in SNR from the autocorrelation method to the crosscorrelation method

$$
\frac{\kappa_{a}(\omega)}{\kappa_{c}(\omega)}=e^{-\alpha(\omega) L}
$$

This result makes perfect sense because the reflected term has to travel the entire length of the cable to get back to the receirer. For longer cables this loss can be so significant that the reflected pulse is not detectable.

The received signals used to calculate the cross-correlation function have noise and, similar to the autocorrelation method, the noise correlation function superposes with the desired correlation function. If the noise is wideband thermal noise, generated in the receivers, there is no added term because the noise terms in the two channels 


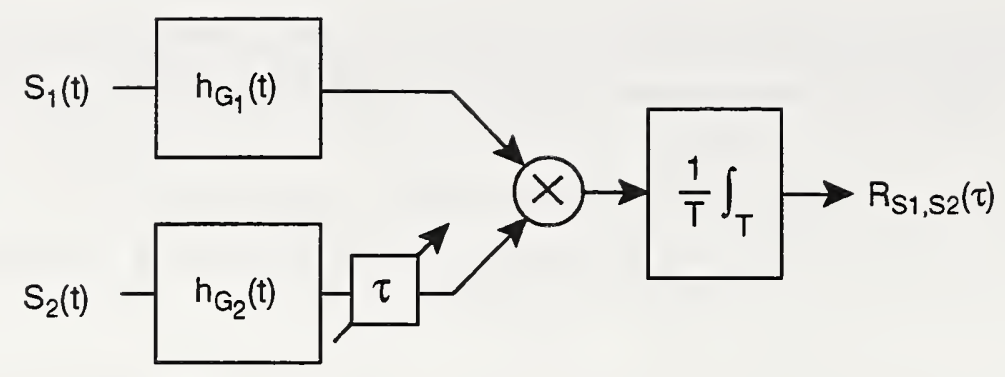

P.D $0-10$

Figure 6-10. Block diagram of generalized cross-correlator showing a prefilter in each channel for eliminating interference.

are independent. When the noise is from external interference entering at the cable terminations, the noise contributes terms at delays corresponding to the cable ends. Just as in the autocorrelation method, the noise-correlation function can obscure PD sites near the ends of the cable and if strong enough can obscure sites located far within the interior of the cable.

Cross-correlation can be combined with a variety of noise-reduction techniques to enhance its performance in the presence of external interference. Using filters in both channels similar to the one in Eq. 6-67 yields a correlator that is referred to as the Wiener generalized cross-correlator (Hero, 1985). A block diagram of the generalized cross-correlator is shown in figure 6-10. The prefilters are used to eliminate undesirable interference in each channel prior to cross-correlating. The filter in each channel is given by

$$
H_{G}(\omega)=\frac{\overline{a^{2}}|S(\omega)|^{2}}{\overline{a^{2}}|S(\omega)|^{2}+N(\omega)}
$$

This type of correlator is especially effective in eliminating narrowband interference from radio broadcasts. An example of the efficacy of this approach is shown in figures 6-11 and 6-12. The result in figure 6-11 shows the cross-correlation function of two PD pulses (one received at either end) corrupted by correlated, multitone, narrow-band interference. The presence of the PD site is obscured. If these same signals are prefiltered prior to cross-correlating, the interference in nearly eliminated and the PD site is easily identified as can be seen in figure 6-12.

The main drawback of cross-correlation is the requirement that both ends of the cable be instrumented. The instrumentation at both ends must be time-synchronized which requires a communications link between the two ends. This communications link can easily be provided for using a low-loss coaxial cable. As an alternative it might be possible to use one of the other cables in the tray that contains the cable under test. However, in this case the other cables in the tray may be just as lossy as the cable under test, so there will be no increase in SNR. Despite this limitation, cross-correlation remains an attractive choice because of the variety of noise reduction 


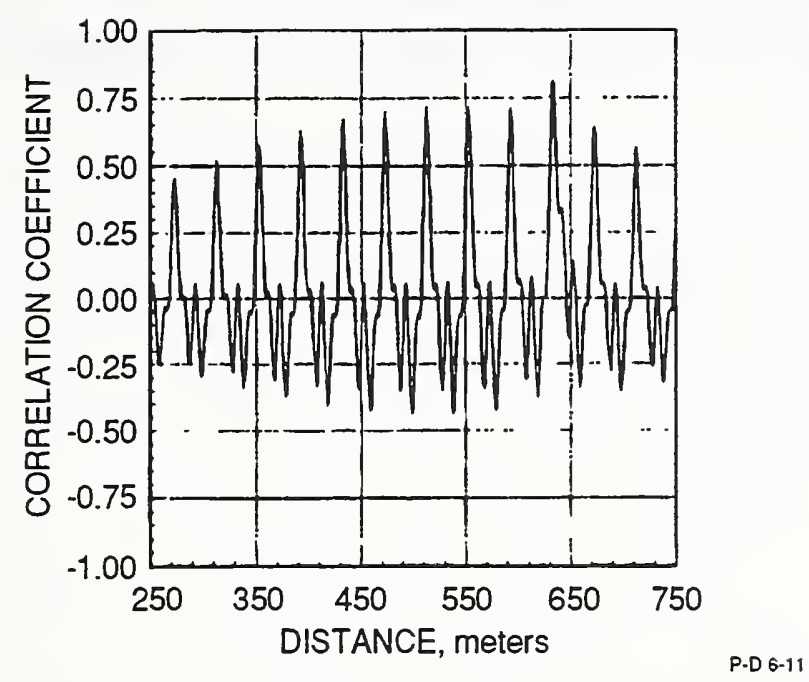

Figure 6-11. Cross-correlation function of a partial discharge corrupted by narrowband interference. It is nearly impossible to identify the partial discharge site.

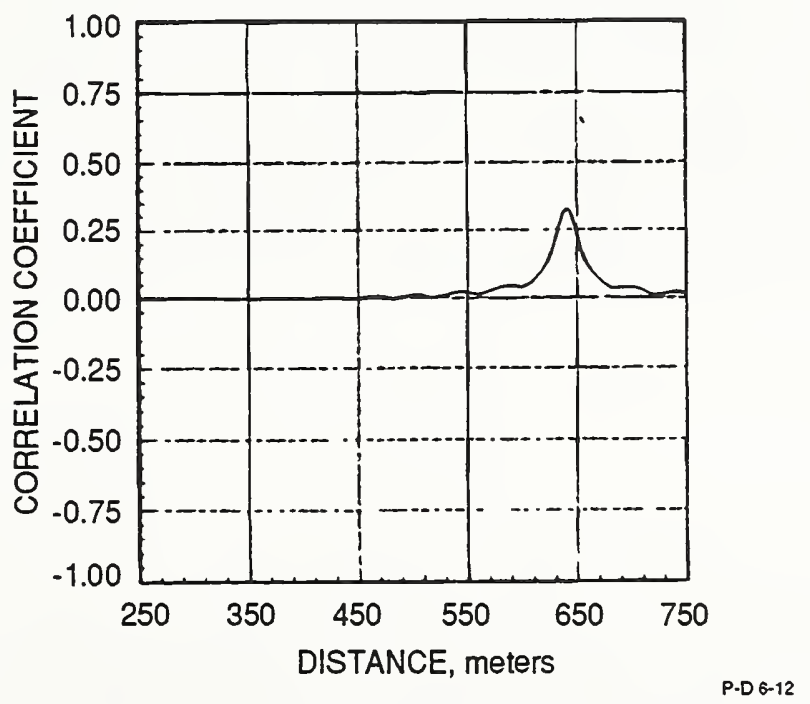

Figure 6-12. Cross-correlation after pre-filtering. This is the cross-correlation function of the same partial discharge corrupted by narrowband interference in figure 6-11. Note the efficacy of prefiltering the signals prior to cross-correlating. 
methods that can be easily incorporated, in addition to its superior resolution, bias reduction, and dramatically increased sensitivity (with a low loss link). Furthermore, most commercial data acquisition equipment have provision for two channels of input, so that little additional investment is required for data acquisition equipment. 


\section{Experimental Apparatus}

\subsection{Introduction}

The aim of the partial discharge (PD) method is to proride a nondestructive diagnostic tool capable of identifying indiridual flaws in the insulation of a cable. Measurements are made across the terminals of the cable and information is gathered to indicate the presence of faws in the insulation. A high roltage is used to induce PD actirity at the site of the defect. The premise is that sites of defects will hare more pronounced PD activity than other points in the cable. Partial discharges manifest themselves as pulses traveling along the cable under test. The PD method analyzes these pulses to determine both the location of the PD site and statistics of the PD process at that site. Based on these measurements, it is determined whether a significant flaw exists at that point in the cable. The PD method is the only nondestructive method that has successfully identifed localized insulation flaws.

\subsubsection{Circuit disconnection}

Any of the electrical methods, so far identifed as useful, requires that both ends of the cable be disconnected and isolated. In some cases properties of the insulation are measured. If the cable is still connected to equipment at the far end, properties of both the cable and the derice to which it is connected are measured. In almost all cases the derice to which the cable is connected will dominate and usually overwhelm any subtle responses from the cable under test if it is not disconnected. In the case of the PD method any derice connected to the cable might be damaged by the test roltage. Eren if the derice can withstand the roltage levels necessary for testing: there is the possibility that the derice would generate partial discharges that would interfere with testing.

\subsubsection{Automation}

Testing at a plant is a massive undertaking. Even when using an instrument that is expensive, the labor costs involved far outweigh capital expenditures. For marginal extra expense, instrumentation can be designed to be simple to use. While it certainly rould be desirable to hare a fully automated instrument, from a practical point of riew, this may never happen. Howerer, computer-assisted operation is an option. where the computer aids in the interpretation by providing the operator with choices or making the operator select regions of interest. In this manner, the instrument can be applied without requiring a highly specialized, experienced operator. 


\subsubsection{Trend or single measurement}

One of the goals of any instrument is to provide a definitive answer with only a single measurement. The costs associated with disconnecting the cable terminations, maintaining records and actually performing the measurement are high enough that only a small portion of cables could be tested. When making a measurement, the first question that is asked is what does the measurement mean? Is the cable good or is it bad? A baseline measurement is needed. In some cases, the parameter change is subtle and the changes must be tracked over a long period of time so that the entire history of the parameters can be studied. In other cases the results are not subtle and it is immediately apparent to the operator that the cable is bad. It is fair to say however, that maintenance of a historical record would significantly aid in the interpretation of the data.

\subsection{Measurement system}

The measurement system assembled for this project included a high-voltage system, an analog measuring circuit, a data-acquisition system and a computer. The highvoltage system, data-acquisition system, and computer were general purpose items. The analog measuring circuit was specifically designed for this testing.

\subsubsection{High voltage system}

The high-voltage system used for the laboratory demonstration consisted of a test transformer excited by a power amplifier. In the laboratory set up using available hardware, the test transformer was PD free and capable of delivering $5 \mathrm{kVA}$ at $25 \mathrm{kV}$. While the power amplifier was capable of generating other frequencies, tests were made at $60 \mathrm{~Hz}$. During testing, the high-voltage transformer output was raised to the test voltage and maintained at that level until the data collection was complete. The voltage was then removed from the specimen. The initial voltage level was selected to be $20 \%$ above the level at which partial discharge first appeared in the cable. The inception voltage was defined as that voltage necessary to produce more than two $\mathrm{PD}$ pulses per second in excess of $10 \mathrm{pC}$.

\subsubsection{PD signal processing system - Initial design}

Residual noise from partial discharge in the transformer and noise on the mains can couple through the test transformer. To minimize the disturbance to the test a lowpass filter is used to condition the test voltage. The circuit of the low-pass filter uses Radio Frequency (RF) quality components and shielding to prevent noise from 


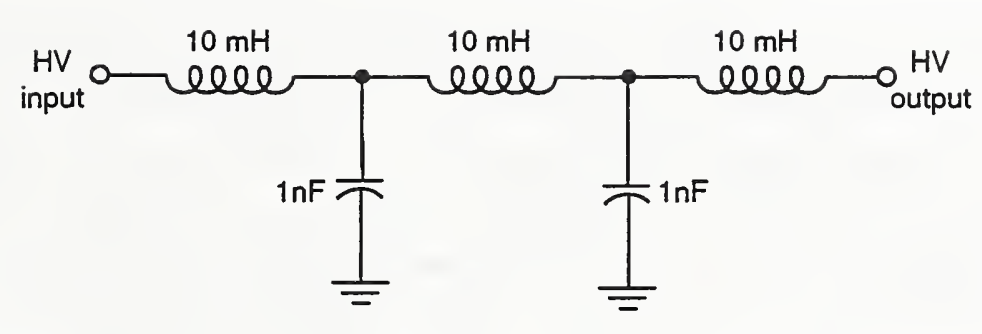

P.0 7.1

Figure 7-1. Lowpass filter for high-voltage power supply

coupling into the cable under test. The low-pass filter is shown in figure 7-1. This particular topology is used so that the output element in the filter is an inductor. The inductor causes the filter to appear as a high impedance to the cable under test. The alternative configuration uses a capacitor as the final element and would shunt the signals of interest to ground. With an inductor as the outout element in the ladder network, the impedance looking back into the power supply is essentially an open circuit in the measurement bandwidth.

Initially, the measuring circuit was designed to detect narrow, low-level PD pulses, that is, broadband signals. A preamplifier was located at the high-voltage detection network to minimize the noise. The detection network was designed to present a matched impedance to the cable under test while passing the signal of interest in an undistorted fashion.

There is always a tradeoff between fidelity of the signal and the physical dimensions of the network used to measure it. The best design uses elements which have nearly ideal characteristics across the measurement bandwidth. In general, as the frequency is increased, the smallest dimension components have the most ideal behavior. In the frequency range of interest it is possible to use nearly ideal components in most of the filter network. The exception is the high voltage capacitor. Almost all of the $60 \mathrm{~Hz}$ high voltage is attenuated in this first element of the filter, the high voltage capacitor. As a consequence, this capacitor must be rated to withstand this voltage. The capacitor must have good RF characteristics. Parasitic inductances in the capacitor can dramatically limit its bandwidth and severely distort the signal. Low-inductance capacitors have an extended foil design and are traditionally used as pulse capacitors. On the other hand, this capacitor must be PD-free at the voltages used in the test. Most PD-free capacitors use a tab foil design to minimize the electrical stress at the capacitor section interconnects. The tab design, by nature, is highly inductive and completely inappropriate for this type of measurement. PD capacitors with an extended foil design become extremely large, even for small values of capacitance. Also, pulse capacitors are designed for dc operation and large pulse voltages, not for continuous ac operation. As a consequence, when operated with continuous ac excitation, pulse capacitors have a tendency to overheat. Both of these constraints require that the pulse capacitor be rated at four to five times the expected peak ac voltage. 
As the capacitor and its surrounding structure becomes large, the return path for the RF currents becomes a significant contributor to the signal distortion. To minimize the distortion, a good return path must be provided. This is most easily accomplished using a coaxial geometry for the high voltage capacitor. Providing a coaxial ground return for the $\mathrm{RF}$ signals can increase the undistorted bandwidth by nearly an order of magnitude, provided that the capacitor has similar low-inductance properties. The coaxial shield around the high-voltage capacitor has an additional advantage; it provides additional noise immunity. The loop formed by the cable termination into the measurement circuit is a major contributor to the susceptibility of the system to radiated noise. The coaxial shield minimizes this noise coupling path. There is a disadvantage also: if not properly designed, this shield can become a source of partial discharge which masks the partial discharges of interest.

For purposes of delay estimation, the best design has a filter network that provides a good radio frequency ( $R F)$ path for the signal and a matched impedance over the entire measurement bandwidth. The original design used a coaxially mounted $1 \mathrm{nF}$, $25 \mathrm{kV}$ pulse capacitor as the first element of a $50 \Omega$, Gaussian high-pass filter. This provided a $50 \Omega$ termination impedance for the cable under test in a passband from $850 \mathrm{kHz}$ to $100 \mathrm{MHz}$. The termination circuit also included a low-noise preamplifier with a gain of 5 and noise figure of $6 \mathrm{~dB}$. This gave the system sensitivity of nearly $0.01 \mathrm{pC}$ on the highest gain setting. For short cables, this design was nearly optimum. However, for longer cables the signal attenuation is so severe that much of the available energy is lost. Initially, it was thought that only very small partial discharge would be present, so no method was included in this circuit to attenuate the received signals prior to pre-amplification. When large PD pulses were received the amplifier would compress the pulse and in some cases severely distort it. After the first few experiments it was evident that only large PD pulses are produced by the defects of interest.

\subsubsection{PD signal processing system - Final design}

Two fundamental changes were made in the receiving circuit. First, the design was modified by changing the lower cutoff to $30 \mathrm{kHz}$. This change was accomplished by reducing the termination impedance to $2000 \Omega$, which increased the lower frequency content, providing the needed energy in the received signals but eliminating the desirable matched impedance at the termination. To maintain a matched impedance at the termination would have required a larger capacitor which would have increased the power requirement beyond that available from the test transformer at the National Institute of Standards and Technology (NIST) facility. An alternative approach would have been to reduce the excitation frequency by using a very low frequency supply; however, this type of supply was not available. 


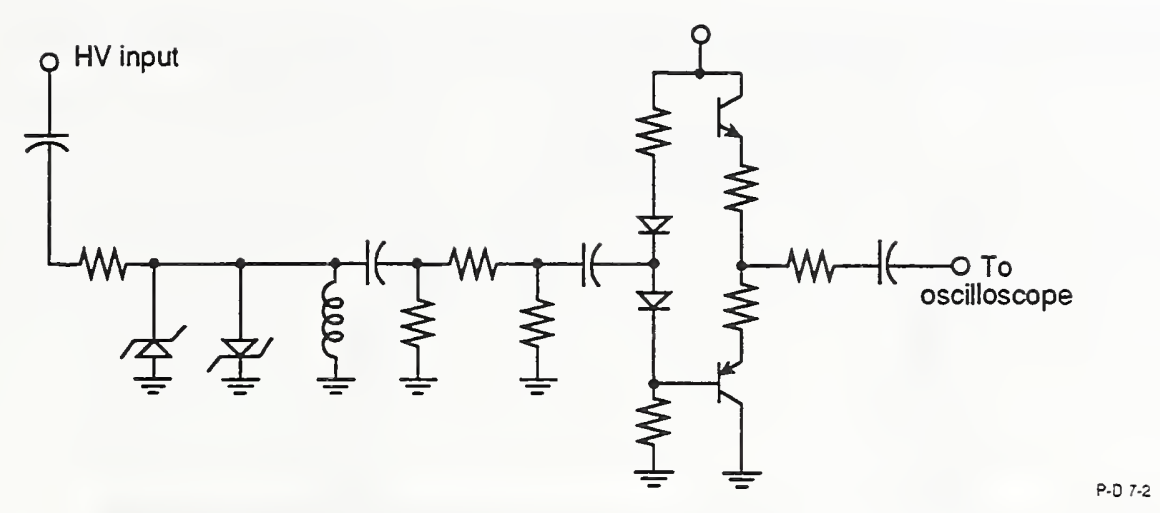

Figure 7-2. Circuit diagram and component values for the receiving circuit

The second fundamental change in the circuit was to change the preamplifier from an amplifier with voltage gain to an amplifier with current gain. A push-pull emitter with a large bias was used to increase the dynamic range of the receiver since large partial discharge pulses were expected.

To prevent accidental damage to the instrumentation from sample breakdown, silicon avalanche protection diodes were included in the network terminating the cable. These diodes are shunt elements and have a non-negligible capacitance. The combination of the protection diode capacitance and the other network elements resulted in a usable bandwidth of $10 \mathrm{MHz}$. This bandwidth was deemed acceptable because most of the cables under test had a smaller bandwidth than this. A fast-rise impulse generator with a $15 \mathrm{~ns}$ rise-time and $100 \mathrm{~ns}$ pulse-width was used to test the integrity of the circuit with impulse voltages of $10 \mathrm{kV}$.

Most of the discharge activity of interest was in excess of $10 \mathrm{pC}$ and the noise characteristics of the circuits were well matched to this limit. Using a larger termination impedance substantially increases the thermal noise generated by the network. In addition, the preamplifier in the circuit was for current gain, not voltage gain and used generic (noisy) transistors, contributing further to the thermal noise. Extraneous, high-frequency noise outside the measurement band was eliminated. With this circuit the sensitivity of the measurement system was limited to $0.5 \mathrm{pC}$.

The termination point of the cable into the detection network was a particularly troublesome portion of the system. The cable was connected into the network as shown in figure 7-2. The wires were spread apart to make the transition into the detection network. The electric field was enhanced at the point were the wires entered the jacketed portion of the cable. The enhanced electric field caused partial discharge at this point. Whenever cables are tested for $\mathrm{PD}$, the termination point represents potential noise sources and care must be taken to eliminate the noise.

Typically, high-voltage power cable terminations are specially prepared using highresistance coatings or high dielectric constant materials to reduce the stress at the termination point. Several alternate methods were tried in an attempt to minimize 


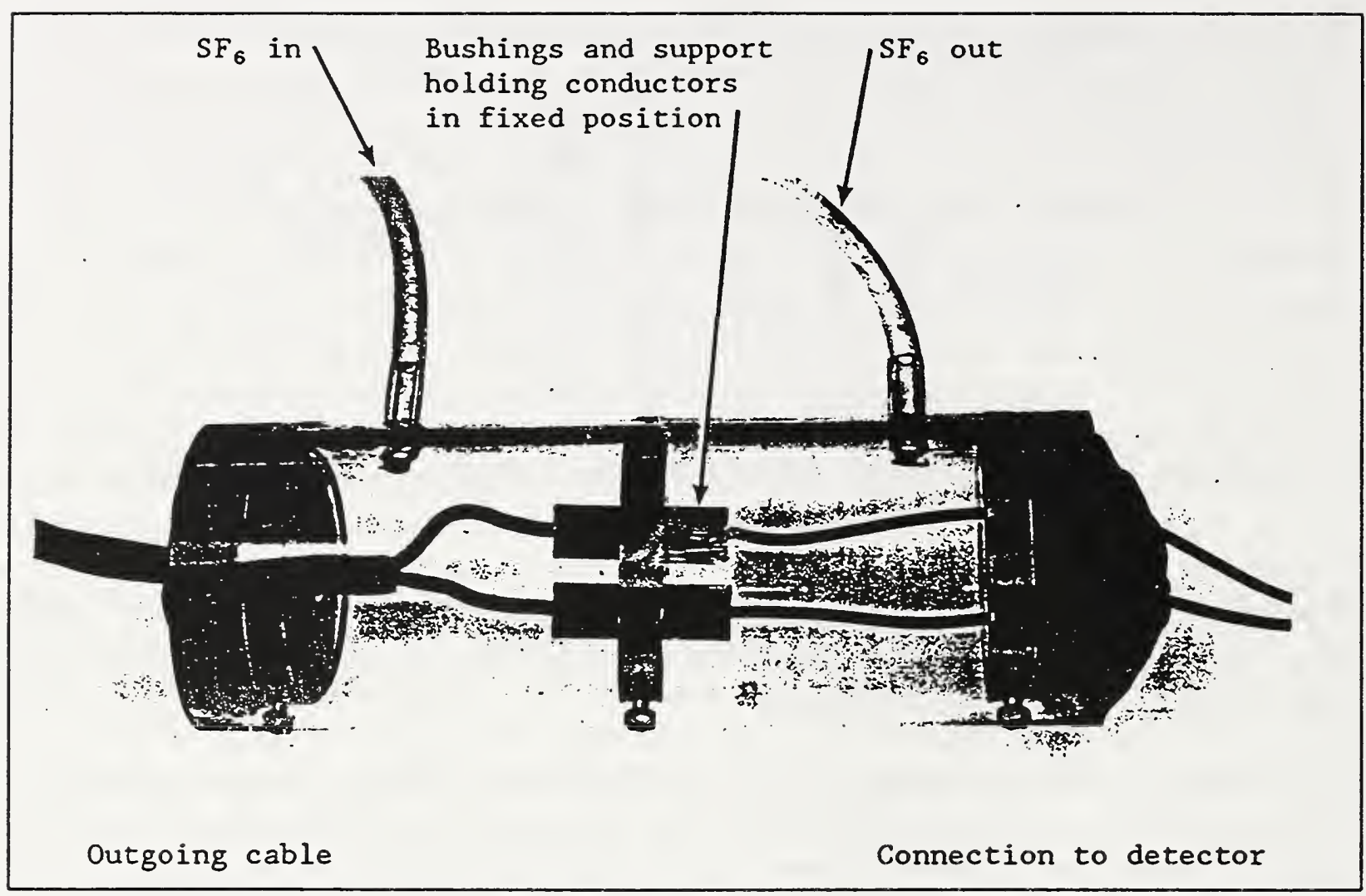

Figure 7-3. Sleeve installed over two-conductor cable to minimize PD at the splice

the partial discharge at the termination. The first alternative was to pack silicone putty around the transition point. The idea was to fill the air space with a material having a dielectric constant closer to that of the cable, in effect reducing the stress. In many instances this method reduced the partial discharge but did not eliminate it. Another alternative was to fill the space with an electronegative gas with a high dielectric strength. Sulfur hexafluoride was used in a special, sealed termination shown in figure 7-3. The principle of this approach was to use a gas which reduces the probability of ionization. Once again, this method reduced the amount of partial discharge but did not eliminate it. The most effective (but impractical) method was to immerse the termination in oil. The oil effectively eliminated the air gaps causing the partial discharge.

\subsection{Data acquisition system}

The data acquisition system played a key role in the success of the method. From a statistical standpoint, a large number of measurements are needed for a reliable result. Identification of the defect sites relies upon interpretation of estimates of the probability densities of the partial discharge. Estimates of the probability densities 
are made using histograms which require many thousands of data points and each PD pulse gives only one data point.

The requirement for a large number of records places demands on the data-acquisition system. Typical data-acquisition systems acquire a single waveform and then transfer the waveform to a computer. The software overhead associated with this transfer is enormous. While the actual transfer of data can be accomplished rapidly, the overhead associated with the transfer limits the throughput of the system to a few transfers per second. Each data record of a single PD pulse comprises anywhere from 500 samples to 2000 samples.

Another problem is that of capturing all PD events. If the data-acquisition system is busy transmitting data on a PD pulse to the host computer, it can miss subsequent events. The digital oscilloscope used in these measurements has a special architecture designed to alleviate this bottle-neck. The high-speed memory associated with the data acquisition can be segmented into 250 small sections. When the acquisition cycle begins, the digital oscilloscope is capable of recording up to 250 separate partial discharge pulses before needing to transfer data to the host computer. Associated with this architecture is a dead time used by the acquisition system to reset internal parameters. During this dead time $(250 \mu \mathrm{s})$, the digital oscilloscope does not respond to a trigger. This arrangement minimizes the number of significant events missed by the system. While $250 \mu \mathrm{s}$ is still a long time, it is far superior to hundreds of milliseconds associated with systems that transfer each record before collecting another.

The data are transferred to the host via a IEEE-488* GPIB** bus. The computer software is menu-driven and contains automated archiving features. The data are temporarily stored on a random access memory disk to eliminate any overhead associated with accessing a disk drive before the test is complete. The software contained in the digital oscilloscope limits the transfer speed of the 250 segments to approximately 60 seconds. Typical tests collect 5000 waveforms and require about 20 minutes for this acquisition process.

More recent versions of digital oscilloscopes have increased performance. In these recent versions, the dead time between segments has been reduced to $50 \mu \mathrm{s}$ and host transfer speeds have been reduced to 1 second, but only 200 segments are available. This increased performance would decrease the acquisition process to 25 seconds and dramatically reduce the time that the insulation is stressed with high voltage. Recently introduced digital oscilloscopes have internal ram disks eliminating the need

*IEEE: Institute of Electronics and Electrical Engineers

** GPIB: General Purpose Interface Bus. 
to transfer data to the host computer before the end of the test, further reducing the test time.

Specialized high-voltage control circuitry could easily be devised to coordinate the acquisition system with the test voltage. One 200 -segment acquisition cycle would require less than one cycle of the $60 \mathrm{~Hz}$ voltage, using a more advanced digitizing oscilloscope. This improvement means that each 200 -segment block could be acquired while gating the high-voltage "on" for as little as one cycle. The total 5000 segments could be collected in as few as 25 cycles, or 0.4 seconds, dramatically limiting the stress to the cable.

\subsection{Data processing system}

Once the partial discharges have been captured by the data-acquisition equipment, the pulses are stored in a digitizer and then transferred to a personal computer for storage. The data are converted to a form appropriate for the analysis software, and then copied to another identical computer for analysis.

\subsubsection{Hardware}

The hardware consists of a digital oscilloscope, referred to as "digitizer" (LeCroy 9400A*) and two personal computers (PC) (NEC Powermate II*). A plotter (Hewlett Packard $7470^{*}$ ) is used to plot the results (the plotter is interfaced using the RS232 serial port which is included with the personal computer).

The digitizer was chosen because of its ability to take multiple traces in a single record (or sequence). This ability, combined with a large memory, allows 250 traces to be recorded with 112 points per segment (trace) and $28 \mathrm{k}$ points per sequence. The digitizer also comes complete with an IEEE-488 GPIB and full "talker/listener" capabilities which allows control and data transfer with the PC.

To increase the PC performance, a 80287 math coprocessor was added along with 512 extended memory (used by Asyst*). While these computers have nowhere near the performance of current PC's (with 80386 and 80486 processors) they were above average at the time and, most importantly, had been used in similar experiments up to this point by Weeks and Steiner. A National GPIB PCIII*board was chosen over

${ }^{*}$ Certain commercial instruments are identified in this report in order to adequately specify the experimental procedure. Such identification does not imply recommendation or endorsement by the National Institute of Standards and Technology, nor does it imply that the instruments identified are necessarily the best available for the purpose. 


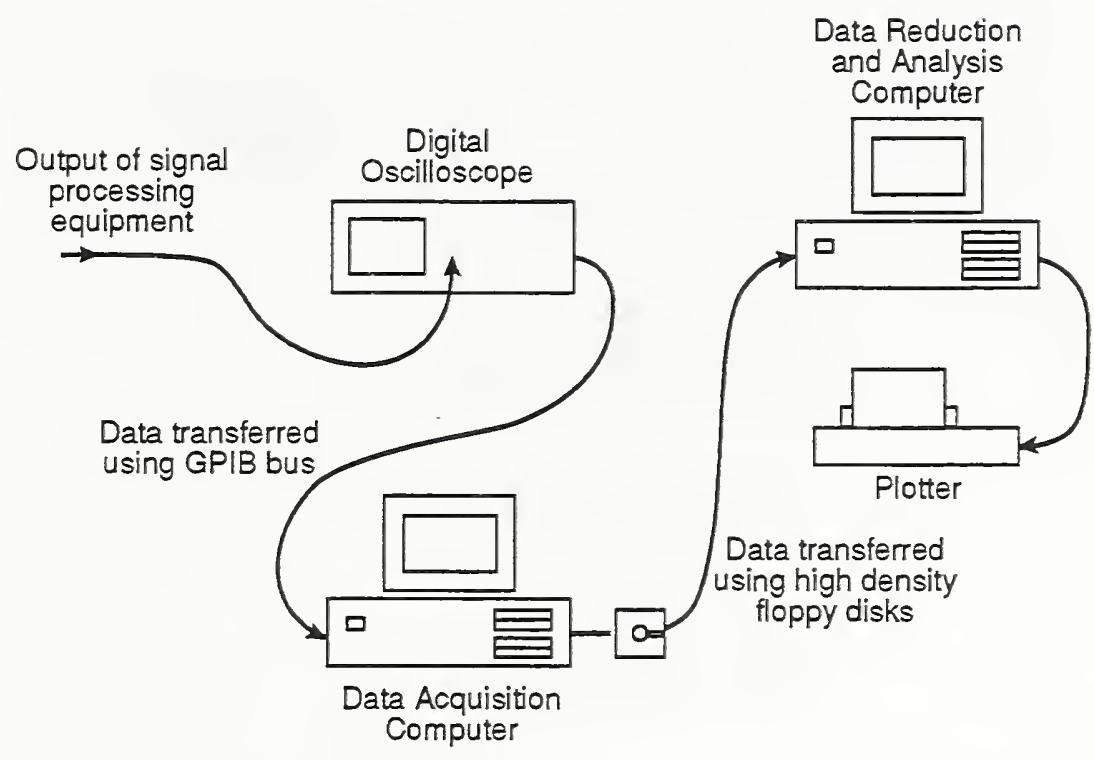

Figure 7-4. Block diagram of the PD analysis system

the standard PCIIa due to its $1 \mathrm{Mb} / \mathrm{s}$ transfer rate and compatibility with the Asyst software package (figure 7-4).

\subsubsection{System software}

\subsubsection{General}

The basic software tool for processing the data is the Asyst program, adapted for a menu-driven, customized process that provides a consistent and user-friendly test procedure. The software consists of three compiled Asyst programs; each fulfills one of the basic steps involved in the test procedure, as shown in the flowchart of figure 7-5. The first step is the transfer of data from the digitizer to the computer and the conversion from the digitizer data format to a format suitable for use with Asyst. The second step involves reducing the data from a waveform into two values per discharge (a signed charge and position). The third step is the analysis of the charge and position values by examining the pulse-height histogram, the position histogram, and using the experience of the operator to analyze mainly the anomalies in the data until the cable defects become clear. Asyst is a high level language program that has many features useful for this application:

- allows use of expanded memory

- high level GPIB command set

- functions for performing histograms 


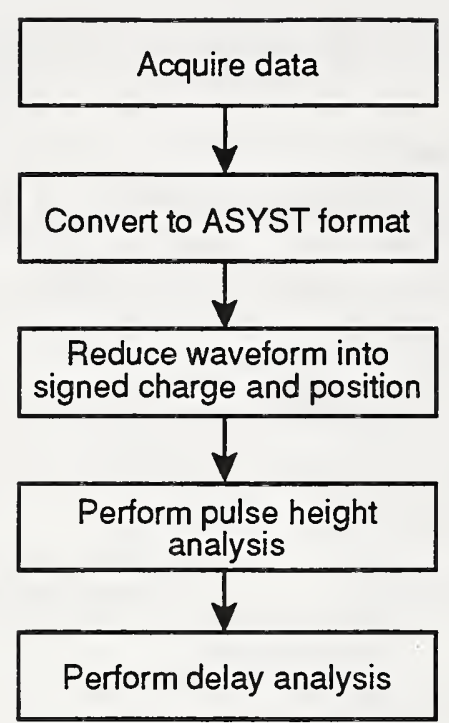

P.0 7-5

Figure 7-5. Flowchart of $\mathrm{PD}$ detection

- functions for creating menus

- functions for generating graphs

\subsubsection{Asystdaq}

The first program is called Asystdaq (for Asyst Data AcQuisition). Asystdaq controls the interface to the digitizer and the data format conversion once the trace information has been transferred to the PC. Figure 7-6 shows a block diagram of the program flow.

The program itself is menu-driven with six steps, as shown in figure 7-7. The first step is the PARAMETER menu, as shown in figure 7-8. The PARAMETER menu has three different items. The first menu item is the NUMBER OF RECORDS (per iteration). This item is the number of sequences the digitizer acquires before transferring them to the PC. A sequence is several successive discharges as recorded by the digitizer in sequential mode. The maximum NUMBER OF RECORDS which can be stored before a transfer is 10. The next item on the menu is the NUMBER OF ITERATIONS, or the number of times Asystdaq performs the above data transfer. The final item is the DISK SIZE for archiving the acquired (and format-converted) data. If low density $(360 \mathrm{kB})$ diskettes are used, 8 are required for the standard 5000 trace group. If high density (1.2 MB) diskettes are used, the group only requires 2 diskettes. The DISCRIMINATION TYPE function is not presently implemented.

Now that all the necessary parameters are set, the oscilloscope must be INITIALIZED. The GPIB bus must first be initialized. Once the bus is initialized and communication with the scope is possible, the digitizer is prompted for information on the waveforms 


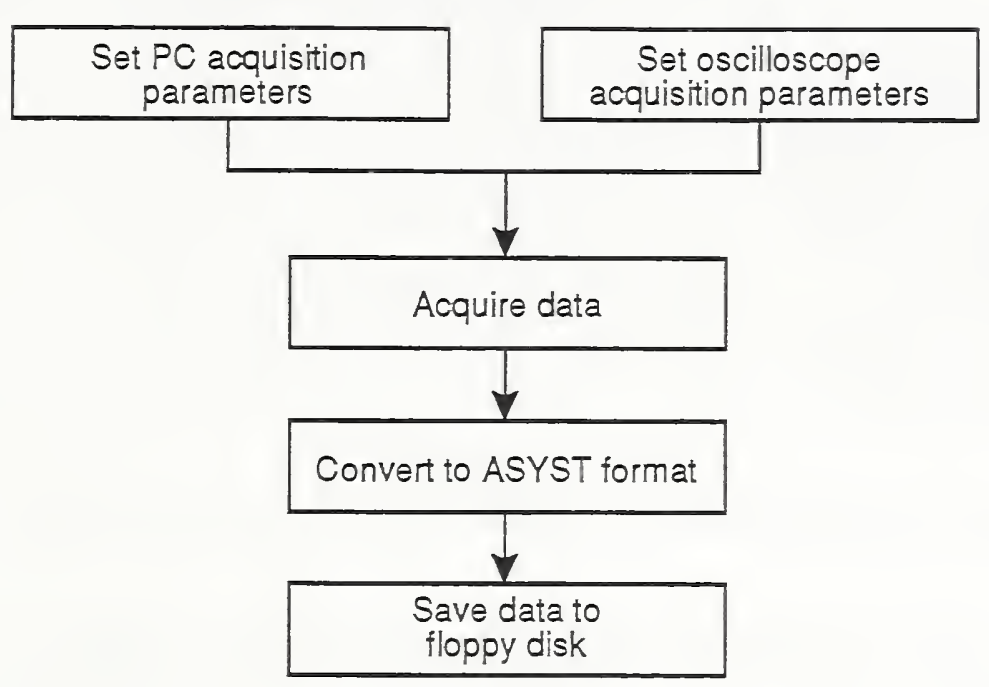

Figure 7-6. Block diagram of Asystdaq

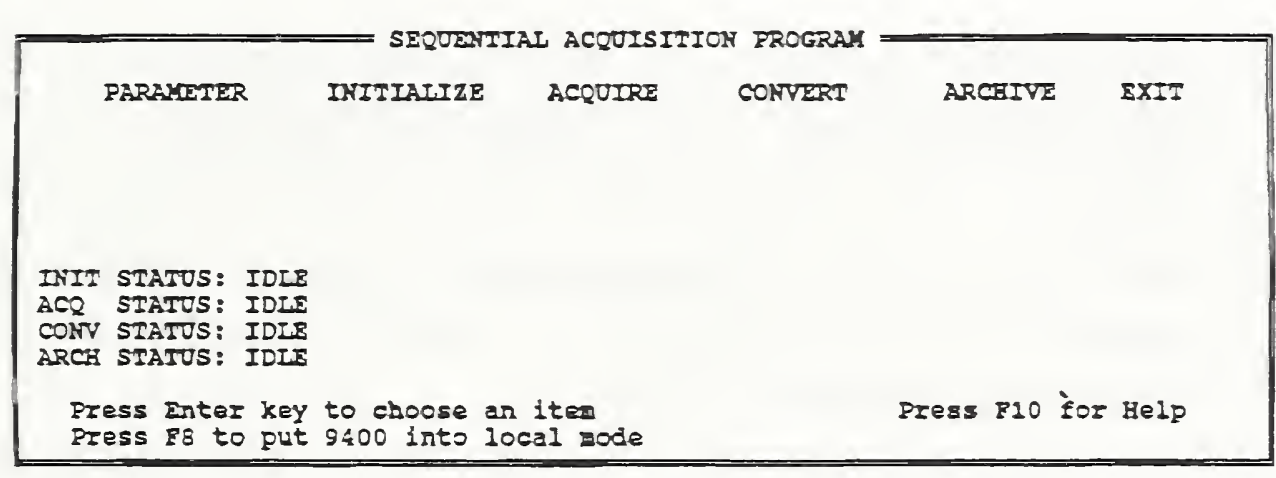

Figure $7-7$. Asystdaq main menu (screen dump)

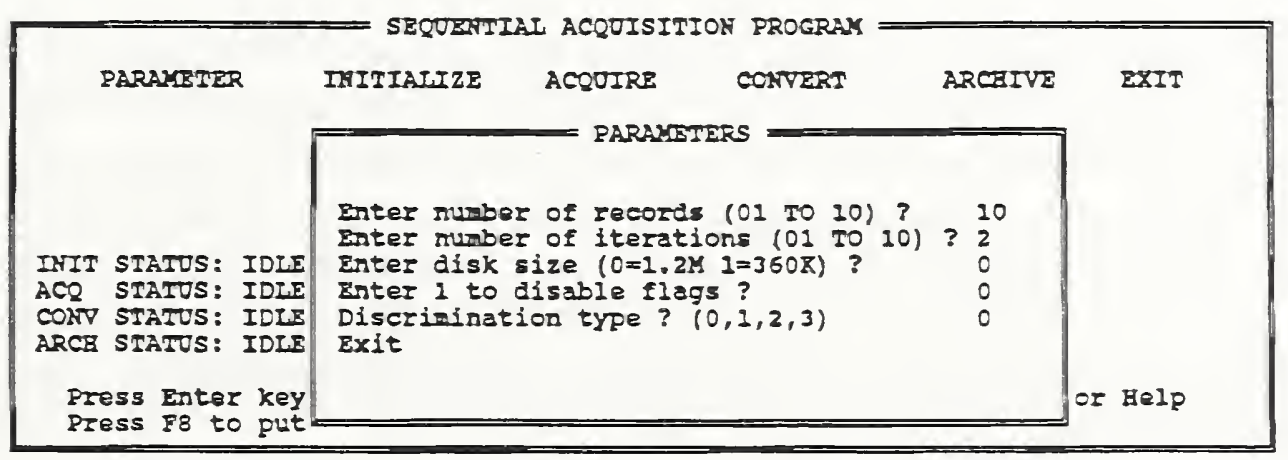

Figure 7-8. Asystdaq parameter menu (screen dump) 


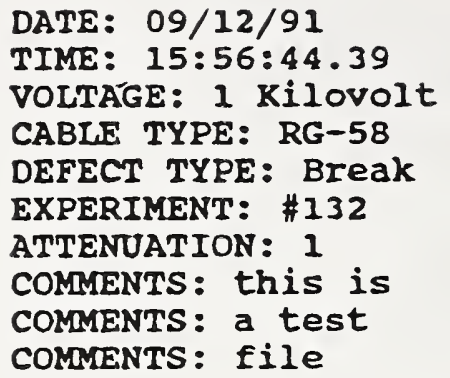

Figure 7-9. Asystdaq RECORD CONTENT (screen dump)

to be transferred. This information consists of array size (amount of data which will be transferred per record) and a descriptor which has the information necessary to scale the binary data transferred by the oscilloscope. Using this information, Asystdaq initializes multiple 'tokens' (a token is a type of Asyst array which can use the expanded memory present in the PC) which will hold the data once the data transfer begins.

Once the INITIALIZATION is complete, data may be taken by selecting ACQUIRE. This routine signals the digitizer to begin acquiring data. When the selected NUMBER OF RECORDS have been recorded (this number is set in the main PARAMETERS menu, the default number of records is 10 ), the digitizer sends a signal to the $\mathrm{PC}$ which then transfers the stored waveforms into its expanded memory. This process is repeated 1 to 10 times (default is 2), depending on the NUMBER OF ITERATIONS selected in the PARAMETER menu.

At this point, all the data reside in the Asyst 'tokens' (or arrays) in expanded memory. There are multiple tokens, one for every record or sequence transferred. The data are in raw binary format exactly as transmitted over the GPIB bus. To be useful to the Asyst program, the data must be converted (by the program step CONVERT) into a scaled binary format which is used by Asyst. This conversion is done by taking the binary levels of the digitizer data and scaling them using the voltage scale data contained in the descriptor string sent by the digitizer during the initialization stage. Once the data have been scaled, they are converted into the Asyst binary file format. The Asyst file created has an ASCII section which has values for voltage, cable type, experiment, defect type attenuation, and comments. The user is prompted to enter these values by the program after CONVERT is selected (figure 7-9). In the menu driven system now implemented, the CONVERT step takes about 10 minutes for a set of 5000 elements ( 20 records of 250 events), during which time the digitizer and the $\mathrm{PC}$ are not available for acquiring data.

The last step in the acquisition process is archiving the data. The main menu of Asystdaq's has a selection ARCHIVE. The ARCHIVE step transfers all the files created in CONVERT and stored on the hard disk over to floppy drives. The files are either 


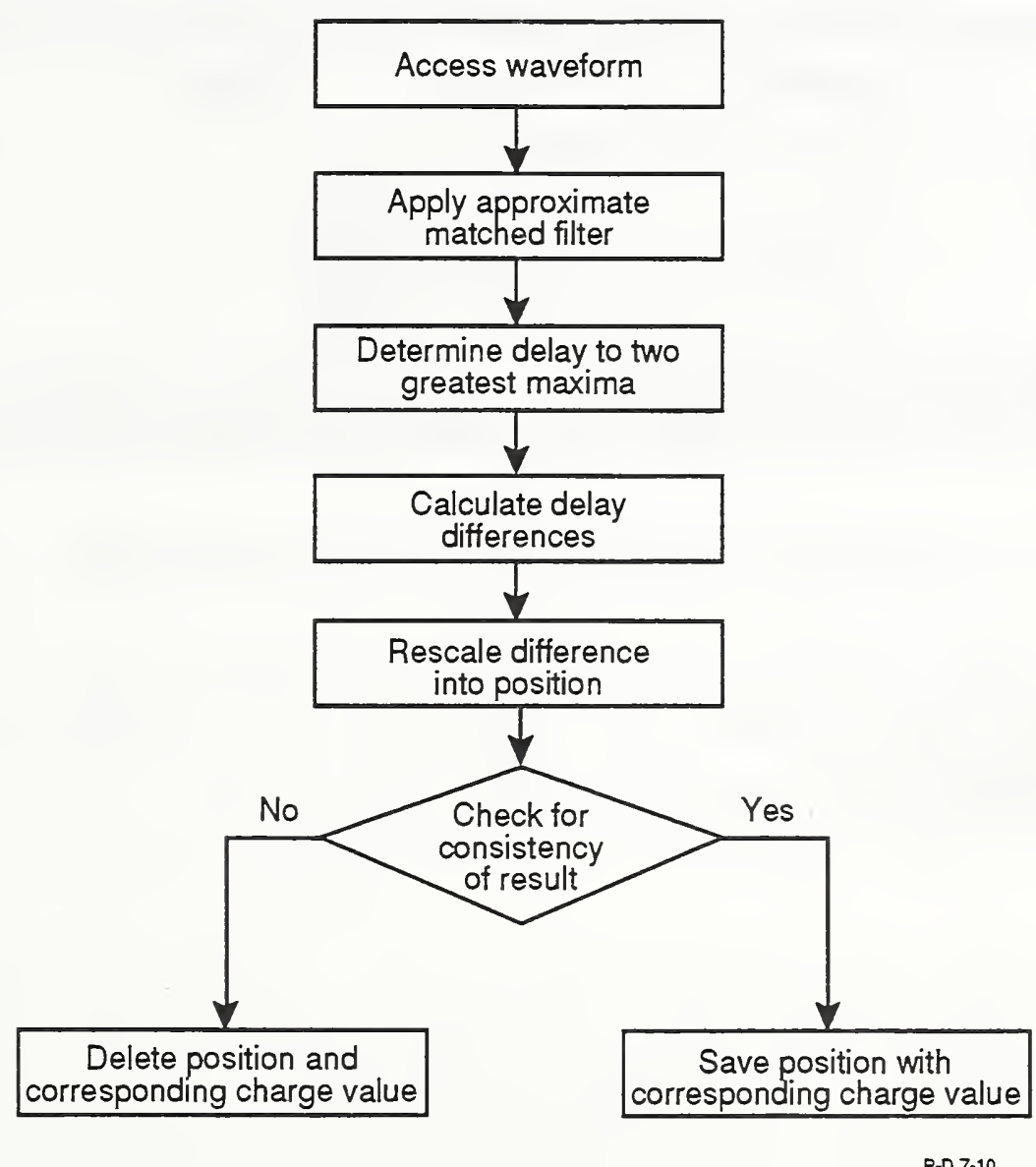

Figure 7-10. Block diagram of Asystred

stored on a $360 \mathrm{kB}$ diskette or a 1.2 MB diskette, depending on what was selected in the parameter section under disk size. Once this is done, the archived diskettes are taken to the second computer for data reduction.

\subsubsection{Asystred}

The second program is called Asystred (for Asyst data REDuction). Asystred is the program which reduces the data to the Asyst data format. Asystred takes the multiple traces collected by the digitizer and reduces each trace to a pair of values. One value represents the estimated amount of charge in the two largest pulses in the trace. The other value represents the distance from the end of the cable to the defect. Figure 7-10 shows the block diagram of the program flow.

The method used to calculate the charge in the partial discharge is one used by Steiner and Weeks (1987), and has been shown to be more accurate than the usual method of peak value. In essence, the technique consists of calculating the energy contained in the waveform and deriving the charge of the pulse from this energy value. It is 


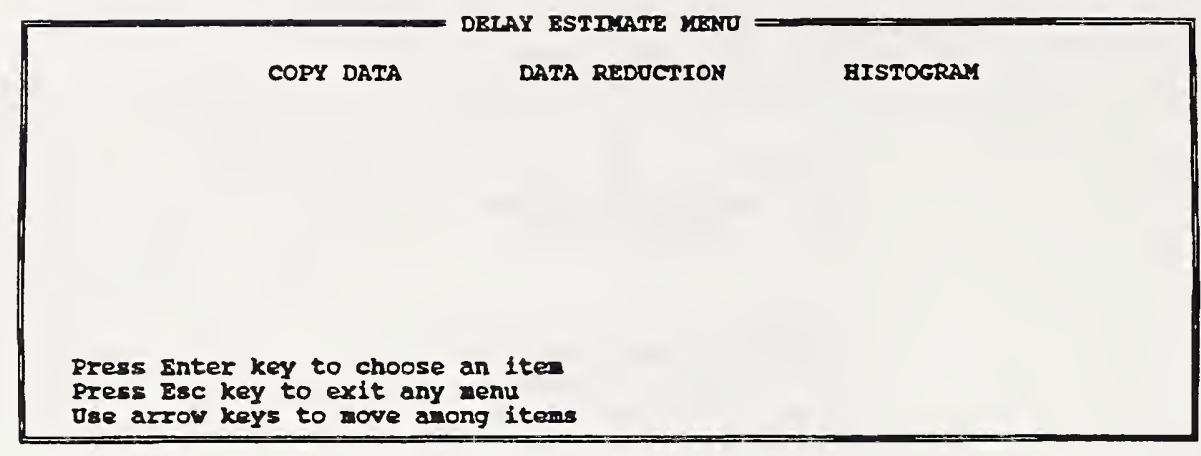

P.D 7.11

Figure 7-11. Asystred main menu (screen dump)

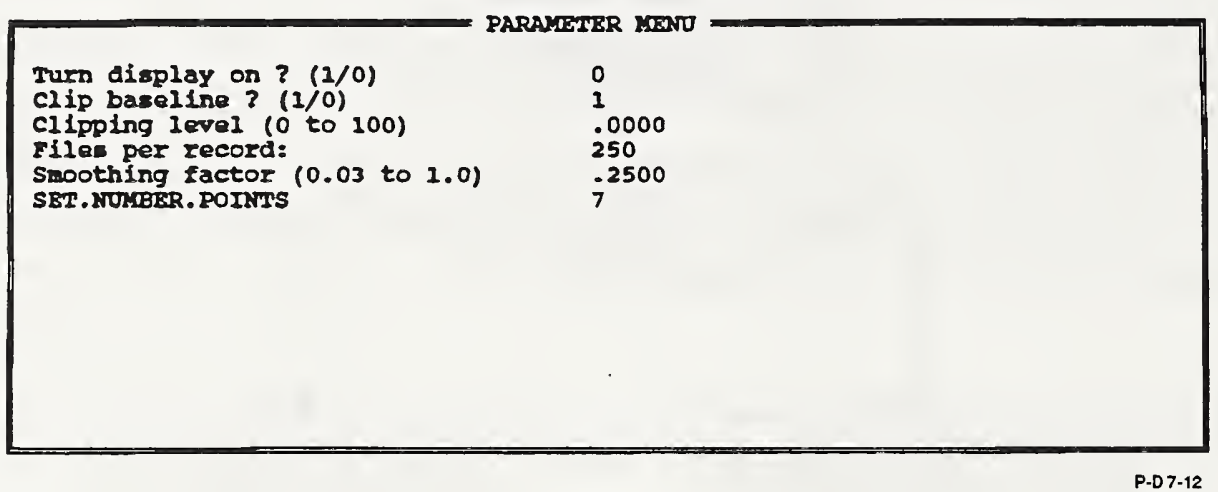

Figure 7-12. Asystred PARAMETER menu (screen dump)

assumed that the defect pulse waveforms are similar to the calibration pulses which are used to normalize the charge data.

When Asystred is run (figure 7-11), the first operation is to COPY the archived files from Asystdaq onto the c: \asy \data directory on the hard drive. When COPY is chosen, a short menu pops up asking whether to CLEAR the SCRATCH DIRECTORY (remove the contents of $c: \backslash$ asy $\backslash$ data), and if the data have previously been reduced (that is, file.dat, not tracexxx.asx). The last step in the COPY menu is EXECUTE. This procedure only takes a few minutes and then the archive disks may be removed. The next menu choice is DATA REDUCTION.

Once DATA REDUCTION is selected, another menu appears. The first choice on the DATA REDUCTION menu is PARAMETERS (figure 7-12). Choosing PARAMETERS lets the user set several options which control the actual data reduction. The first choice on the menu is CLIP BASELINE. The CLIP BASELINE function allows the user to remove pulses under a set level to reduce the effect of noise in the system (the pulse values are taken as the absolute value). If this option is chosen, the user then selects the CLIPPING LEVEL, which is the percentage of the maximum pulse voltage below which level Asyst should remove data. Next, the user sets the amount of traces using FILES PER RECORD. This parameter is set on the digitizer when the data are initially acquired. The SMOOTHING FACTOR parameter sets the 


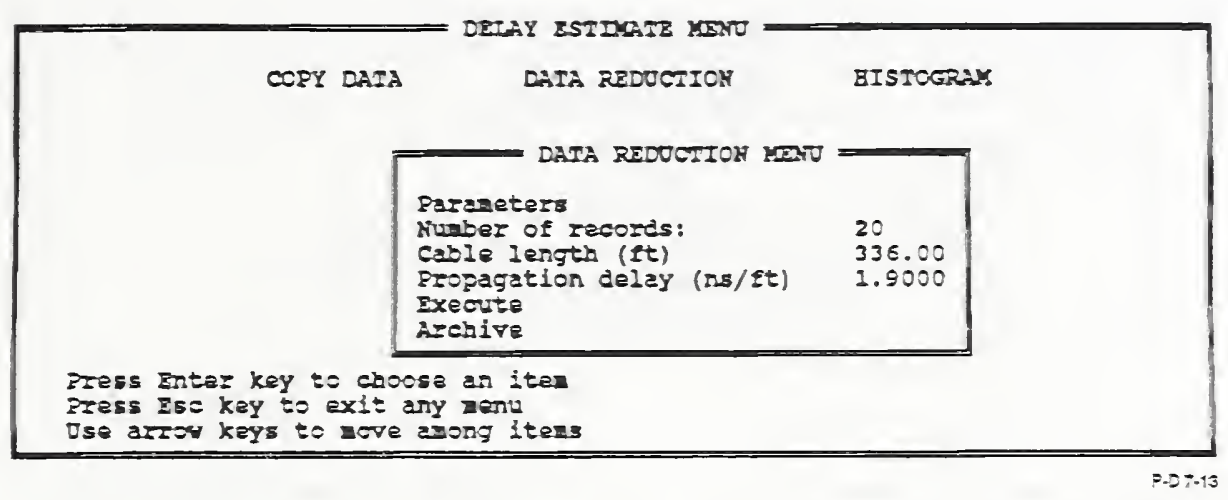

Figure 7-13. Asystred DAIA REDUCIIOA submenu (screen dump)

bandwidth of the approximate matched filter used by Asyst. The last parameter is SET.NUMBER.POINTS; this rariable sets the number of peaks Asyst uses in a preliminary cut before Asyst decides what two pulses are the largest The default is SEVEN, which means Asyst will sort out the seren highest peaks before finding the two highest peaks out of the chosen seven.

After completing the selections in the PARAMETER menu, the user returns to the DATA REDUCTION submenu (figure T-13) and is prompted for NUMBER OF RECORDS (this number is determined by the amount of data originally taken: default is 20). The next selection is CABLE LENGTH. Asyst uses CABLE LENGTH to remore any pulse pairs that are outside of the cable length. By determining the distance from the end of the cable at which the discharge occurred and checking to see if the distance is longer than the cable, Asyst is able to remore some noise. The last choice before executing the data reduction is PROPAGATION DELAY. The propagation delay of the cable is necessary for the program to convert from time units of the delay characteristics into distance units (time $(\mathrm{s}) /$ propagation delay $(\mathrm{s} / \mathrm{ft})=\operatorname{distance}(\mathrm{ft})){ }^{*}$

Once all parameters have been set, Aststred is ready to reduce the data. To reduce the data, the next menu choice, EXECUTE, is selected. Once EXECUTE is selected, Asystred begins to process each data file, displaring the name of the file being reduced on the screen. The DAIA REDUCIION process is rery tedious, taking between 3 and 4 hours to process a typical 20-record data set on an AI type personal computer. The reduced data are stored in the directory $\mathrm{c}: \mid \mathrm{asy} \backslash$ dats in the file: file.dat. When the

*The existing program that was made available to NIST for conducting these experiments in 1989 and 1990 was based on the use of feet as unit of length. Thus, the explanatory figures in this section that are actual screen dumps show feet as unit of length. Typical program-generated plots shown in the explanation of the principle also show length in feet. However, the program-generated plots giving results for specific tests on actual cable specimens in Section 8 have been amended to show both meters and feet as units. 


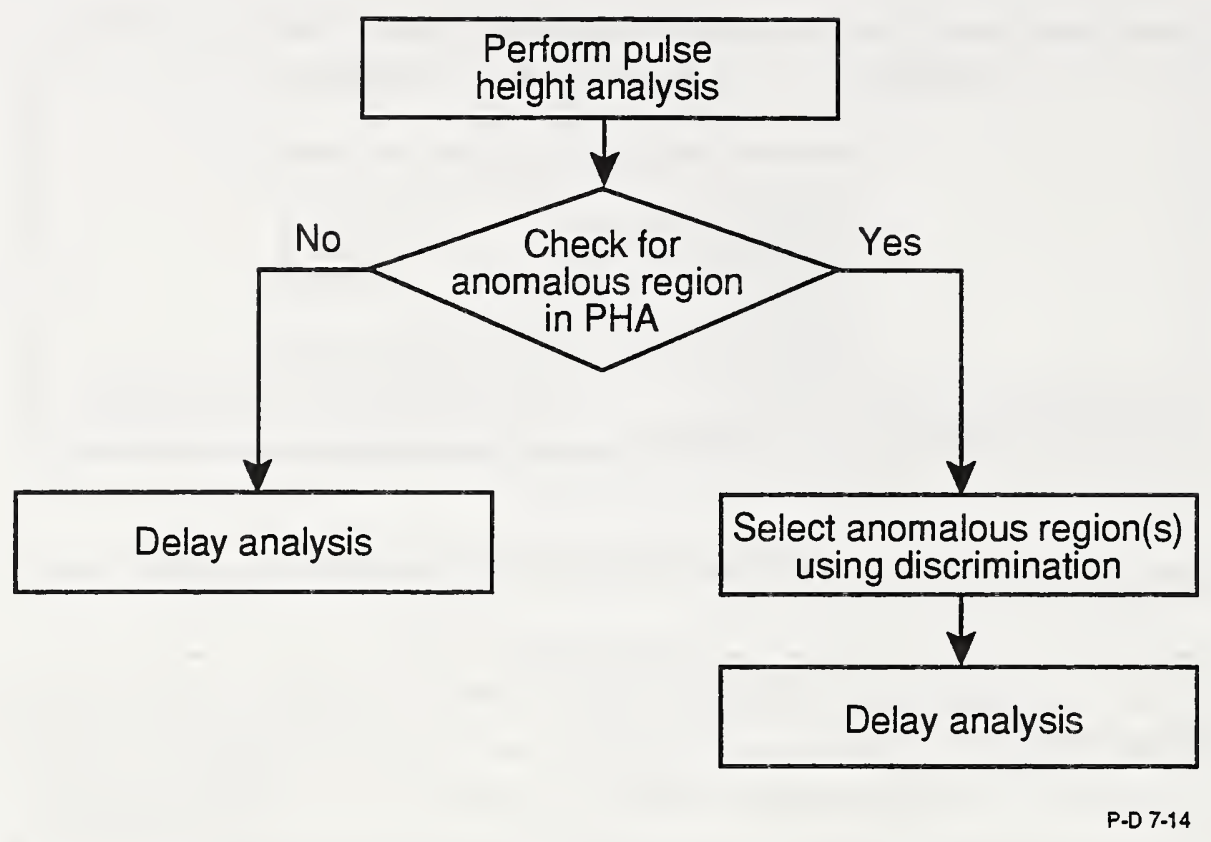

Figure 7-14. Asystanl Block diagram

DATA REDUCTION is done and the file: file.dat is created, the user may ARCHIVE this file to a floppy disk by selecting ARCHIVE.

Though the main function of Asystred is completed at this point, the user may view a HISTOGRAM of suspected partial discharges sites (relative to the end of the cable). This function is mainly used to check if the data followed the form which was expected. The HISTOGRAM function is also part of Asystanl and is discussed in the next subsection.

\subsubsection{Asystanl}

Now that the data have been reduced and the histogram of discharge sites has been checked to confirm that the proper discharge form is present, the data may be manipulated by an expert user to remove some of the noise. The program Asystanl (figure 7-14) allows this to be done to the reduced data file created by Asystred. Figure 7-14 shows the block diagram of the program. Asystanl allows the user to examine the distribution of charges in the cable. Because the distribution of the noise within the cable can be modeled as the distribution of pulses from a partial discharge site, this information can be used to remove noise from the histogram of discharge locations.

Before the PULSE HEIGHT ANALYSIS (PHA) is performed, a CALIBRATION can be run to determine the approximate charge value (figure 7-15). The calibration pulses are generated by applying a fast-rise step of known amplitude to a known capacitance. This pulse is then injected into the open end of the deenergized cable. The calibration 


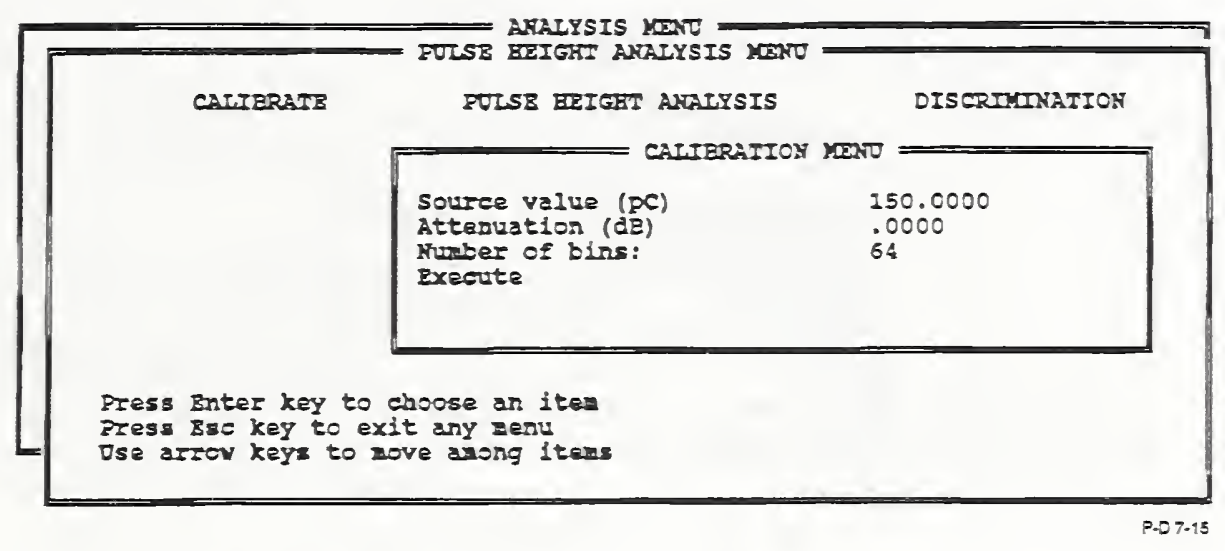

Figure 7-15. Asystanl CAIIBRAIION menu (screen dump)

pulses are recorded using Asystdaq and the data file is then reduced using Asystred. This file is copied onto the hard drive and the calibration procedure within. Asystanl is selected. Once the calibration menu has been selected, the user sets the charge value of the source pulse, the attenuation of the signal processing stage, and the number of bins to use when displaying the histogram. Once these values are set, the calibration can be executed. The program then determines a calibration factor. This calibration factor is a scale factor used to adjust the values of the PD charges when actual data are used. The CALIBRATION routine also displays statistics of the distribution of charges, along with a histogram of the positive and negative charges. While it is possible to make further corrections in the calculation to account for the variation in the attenuation for partial discharges emanating from different locations in the cable, this correction was not deemed necessary at this time. Once the CAIIBRAIION is run, the test data may be loaded in using COPY, then PULSE HEIGHT ANAIYSIS may be run.

The PUISE HEIGHT ANALYSIS involves the sorting of all 5000 pulse pairs (in a standard run) according to their charge as computed in Asystred and normalized by the calibration factor. When Pulse Height Analysis is run, a sub-menu appears (figure 7-16). Here the user enters the calibration factor for the experiment being run. The attenuation of the signal processing equipment is also entered. There are four other parameters to set before executing the pulse height analysis. The DEFAUIT SCALING selection (1/0,1 being on) determines whether automatic scaling is provided. If default scaling is set to 0 (off), then the user may set MAXIMUM VALUE TO PLOT and BIN WIDTH. These parameters determine the scale of the graph and the resolution of the histogram bins. If DEFAUIT SCAIING is set to 1 (on), the resolution of the histogram is set by the next parameter, NUMBER OF BINS.

Selecting EXECUTE will plot two histograms: one histogram for positive charge and one histogram for negative charge. Before displaying the histograms, the program gives some useful information. The first information screen is headed RECORD CONTENTS; this screen shows what file is being accessed (c: $\backslash a s y \backslash$ dats $\backslash$ file.dat) and 


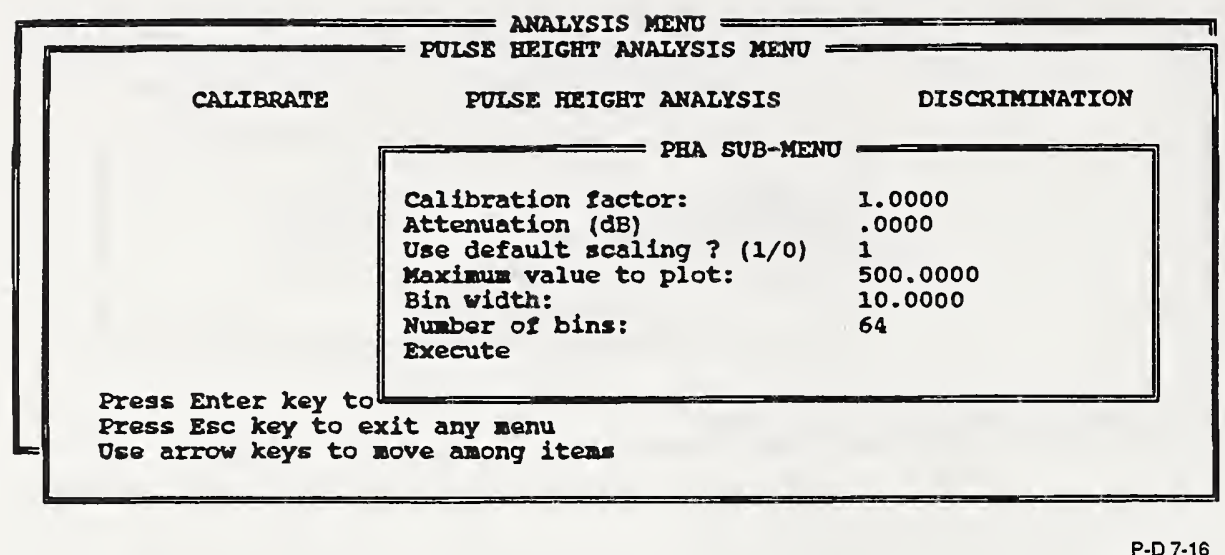

Figure 7-16. Asystanl PHA menu (screen dump)

gives the 10 comment lines entered in the data conversion stage within Asystdaq (date, time, experiment...). The next screen is some statistics of the partial discharge such as mean value, standard deviation, and max-min (figure 7-17). These statistics are given for both the positive and negative pulses. Entering CARRIAGE RETURN after the statistics screen will bring up the HISTOGRAM (figure 7-18). These histograms follow a certain distribution depending on the type of cable and the type and number of defects.

As discussed earlier, the profile of such a histogram in a cable which is free from preferred discharge sites can be expected to be different from the profiles of of cables containing preferred discharge sites (presumably a cable defect). Detecting such an anomaly in the discharge profile can serve as a basis for concentrating further analysis on that part of the histogram. This analysis can enhance the likelihood of isolating the preferred site from the background noise. This selection is an intuitive matter at this moment in the program, but it may be amenable to automated processing through an expert system. Once the expert user has decided what part of the histogram to examine further, the user proceeds to the DISCRIMINATION section of the PULSE HEIGHT ANALYSIS (PHA) menu.

The DISCRIMINATION subprogram lets the user take the histogram of pulse height (as displayed in the PULSE HEIGHT ANALYSIS) and prune the data before doing a DELAY ANALYSIS on the data. The SUBMENU for Discrimination is similar to the submenu for PHA (figure 7-19). The user sets the scale parameters (by hand or automatically). The user also selects whether to examine positive, negative, or positive and negative discharges. When the subprogram is executed, the user is presented with a histogram of discharges (as in PHA) along with two cursor lines. The cursor lines may be positioned using the cursor keys on the right hand side of the keyboard. When the first line is positioned, pressing F9 will allow the other cursor line to be positioned. Typing MARK creates a file called 'file.prn' which contains only the pulses contained between the cursor lines. By exiting DISCRIMINATION 
Pulse Height Analysis Program

Positive Partial Discharge

$\begin{array}{lll}\text { Mean value, positive pd: } & .0168 \mathrm{pc} \\ \text { Standard deviation, positive pd: } & .0017 & \mathrm{pc} \\ \text { number of data points, positive pd: } & 247 & \\ \text { maximum data value, positive pd: } & .0233 & \mathrm{pc} \\ \text { minimum data value, positive pd: } & .0138 \mathrm{pc} \\ \text { number of bins to be used, positive pd: } & 65 & \\ \text { bin width, positive pd: } & .0004 \mathrm{pc}\end{array}$

Negative Partial Discharge

$\begin{array}{lll}\text { Mean value, negative pd: } & -.0156 & \mathrm{pc} \\ \text { Standard deviation, negative pd: } & .0019 & \mathrm{pc} \\ \text { number of data points, negative pd: } & 253 & \\ \text { absolute maximum, negative pd: } & .0232 & \mathrm{pc} \\ \text { absolute minimum, negative pd: } & .0122 & \mathrm{pC} \\ \text { number of bins to be used, negative pd: } & 65 & \\ \text { bin width, negative pd: } & .0004 \mathrm{pc}\end{array}$

To prist screen contents press function key F8, F7 for formfeed type $\langle C R\rangle$ for histogram

Figure 7-17. PHA Statistics (screen dump)

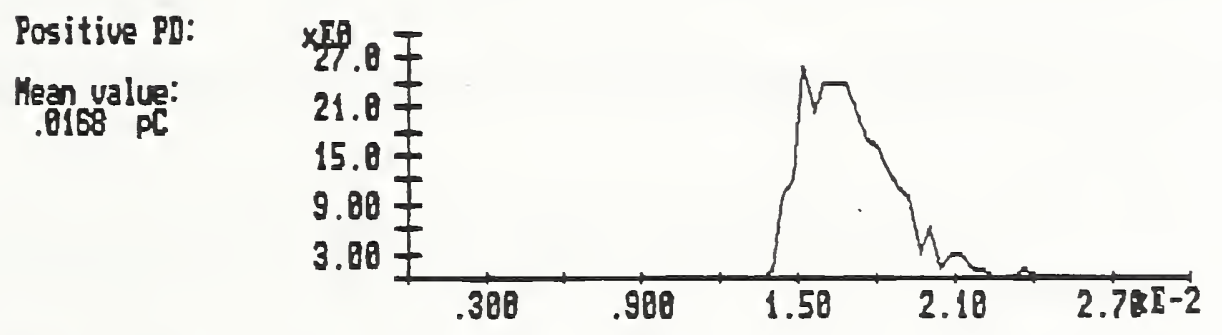

Negative PD:

kean value:

Press 58

to plot

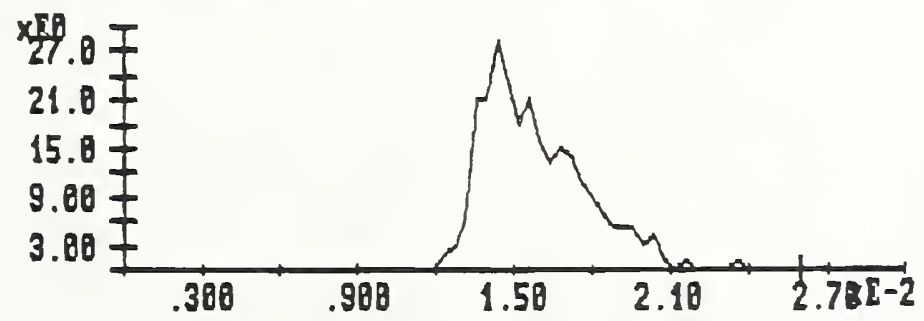

Iype (CR)

to exit

P.D 7-18

Figure 7-18. Asystanl HISTOGRAM (screen dump) 


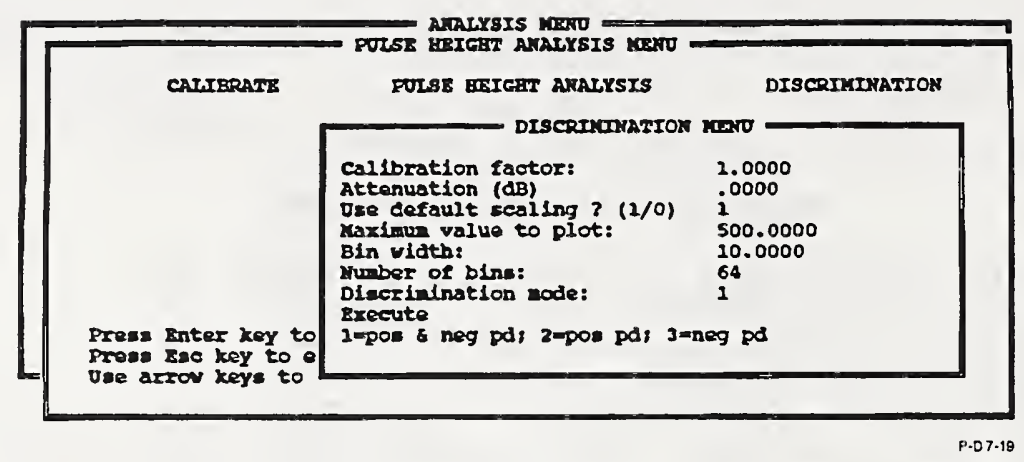

Figure 7-19. Aystanl picture of DISCRIMINATION submenu

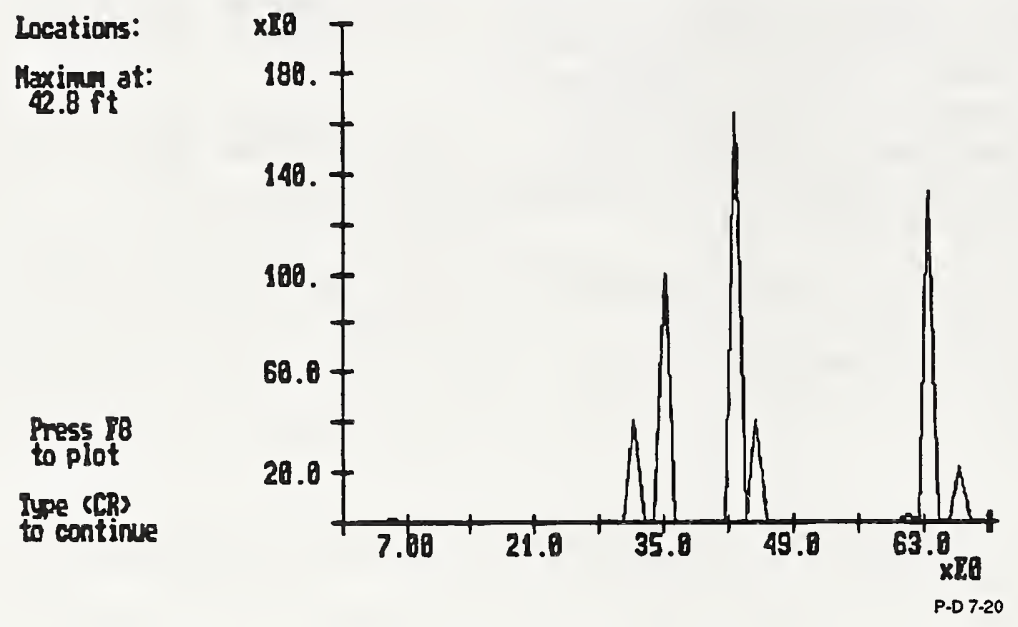

Figure 7-20. Typical Asystanl PD statistics as printed by the program. Horizontal distance axis is printed with feet as built-in parameter.

and PHA and entering DELAY ANALYSIS the user may now examine the histogram of pulses vs distance.

When the DELAY ANALYSIS submenu is entered, there are four parameters related to scaling the histogram; DEFAULT SCALING (1/0), MAX VALUE, BIN WIDTH, NUMBER OF BINS. There is also a question related to the DISCRIMINATION. USE DISCRIMINATION (1/0) will use the file 'file.prn' if 1 is selected and use the file 'file.dat' if 0 is selected. This selection will allow the user to graph a delay histogram of all the pulse data or graph a histogram of just the data pruned using DISCRIMINATION. Once the DELAY ANALYSIS is EXECUTED, the record contents are displayed, followed by the PD statistics. Then the histograms are displayed. They may be plotted by pressing F8, as shown in figure 7-20. Also displayed after the histograms is a representation of the reduced data in tabular form, as shown in figure 7-21. The tabulation yields precise information on the distribution, while the histogram conveys a message of "profile", a useful tool for further analysis. 
HISTOGRAY COUNTS AT CABIS LOCATIONS, PG. 1

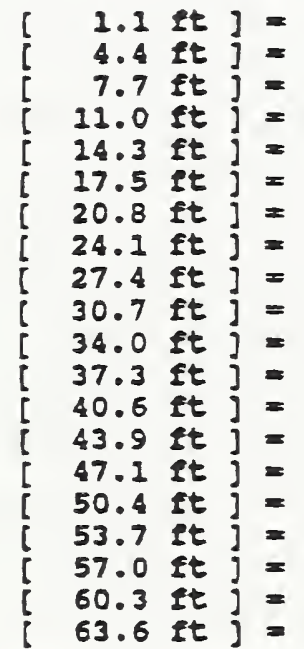

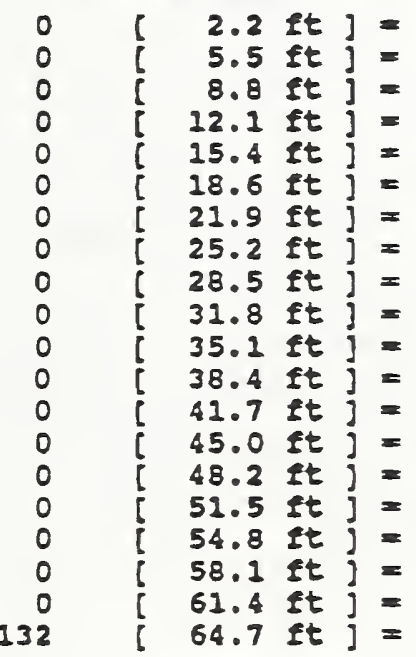

0
1
0
0
0
0
0
0
0
40
99
0
0
40
0
0
0
0
3
0

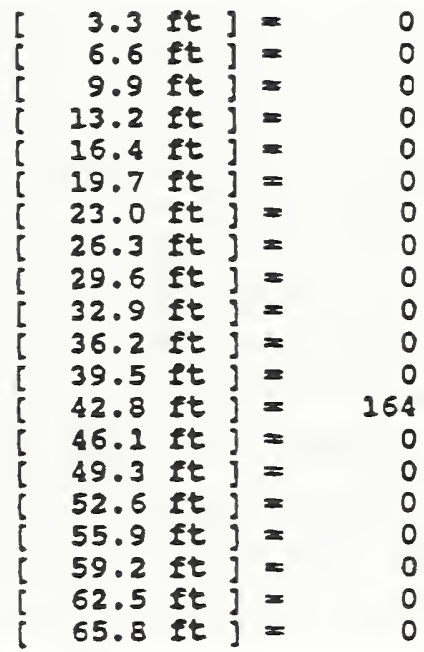

Press 88 to print screen; 77 for form feed; Press < ENTBR > fór next page

Figure 7-21. Typical Asystanl reduced data in tabular form as printed by the program. Horizontal distance axis is printed with feet as built-in parameter. 


\section{Experimental Work}

\subsection{Artificial versus natural defects}

Attempts to obtain cables that had developed known defects under service conditions were not successful, a testimony to the general reliability of cables used in the industry. Thus, the evaluation of the partial discharge (PD) method could be conducted only with artificial defects inflicted upon specimen cables. There is some advantage in using artificial defects that can be controlled at will, as opposed to random and uncontrolled natural defects. However, the nature of the discharges, and thus of the signals, might be different for cables with a natural defect, compared to the cables with inflicted artificially defects that were used in this project.

A desirable, but not yet attained, feature of signal analysis would be to obtain information on the nature of the defect from the characteristics of the signature. If this were possible, more useful information could be obtained from artificial defects in a controlled environment, and the knowledge so acquired could be transferred to the less controlled world of natural defects - whatever they might be. Unfortunately, the state of our knowledge has not reached that point. One problem is the influence of pulse-topulse or phase-to-phase memory propagation that tends to make the PD phenomena susceptible to the effects of nonstationary behavior. Added complications occur in the interpretation of $\mathrm{PD}$-amplitude distributions when the process that controls discharge initiation and growth becomes nonstationary in time. The existence of nonstationary behavior also leads to difficulties in obtaining reproducible data on pulse-height or phase distributions even for relatively simple, well-defined discharge gap conditions.

The existence of memory propagation effects has been verified experimentally for both ac- and dc-generated partial discharge as described in several recent papers (Van Brunt \& Kulkarni, 1989; Van Brunt et al., 1992; Van Brunt \& Kulkarni, 1990; Van Brunt \& Cernyar, 1991). Memory effects arise because of the influence of residuals from previous discharge pulses on the initiation and development of subsequent pulses. The residuals might, for example, be associated with surface charge, ion space charge, or metastable atomic or molecular species that remain in or near the discharge site when the next pulse initiates. As a consequence of these memory effects, there can be strong correlations among successive pulses so that both the amplitude and probability of the occurrence of a PD pulse depend on the amplitude of and time separation from previous pulses. This means that successive pulse amplitudes and their phases or times of occurrence cannot be treated as independent random variables. One consequence of this lack of independence that has not generally been recognized is that the PD pulse-height distributions associated with a particular phase interval of the applied voltage are sensitive to the rate of initiatory electron injection into the discharge site as well as to the local electric-field strength at the site. Small changes in the electron emissivity of surfaces induced by 
the $P D$ activity can result in relatively large changes in the observed pulse-height spectra. This implies that the PD phenomena will tend to exhibit nonstationary behavior, thus making it difficult to precisely define a particular discharge pattern for a specified set of defect site conditions. In fact, there is reason to doubt that one can ever define or control conditions well enough, even in a gap with a relatively simple geometrical configuration, to ensure a particular type of stochastic behavior for the discharge. The inability to obtain reproducibility in the pulse-height spectra of negative-corona pulses in air for a simple point-plane electrode gap under ostensibly identical conditions can be attributed, at least in part, to an inability to gain precise control over the electron emission rate from the point cathode surface (Malik \& Alriny, 1987: Van Brunt, 1991).

Nonstationary behavior of PD phenomena, complicated by memory propagation effects, presents an obstacle to the use of phase-resolved PD occurrence rates or pulse-height spectra for reliable pattern recognition purposes. This obstacle can be partly overcome by measuring conditional pulse-height or phase-of-occurrence distributions (Van Brunt, 1991) that tend to be relatively immune to nonstationary behavior. For example, by determining pulse-height distributions conditioned on fixed time separations between PD pulses, effects due to the dependence of amplitude on time separation can be examined and eliminated, that is, the coupling between these random variables can be effectively removed. The determination of conditional distributions either from direct, real-time measurements or from appropriate statistical analysis of stored data offers the possibility for more refined examination of the stochastic properties of partial discharge and may provide data that is more useful for pattern recognition than the unconditional distributions measured by present systems.

\subsection{Cable specimens}

Four types of cables have been subjected to the PD tests, as follows:

- A two-conductor, \#14 $\mathrm{AWG}^{*}, 600 \mathrm{~V}$ class, neoprene-jacketed, unshielded cable of the type used by Georgia Power Company in their nuclear power plants.

- A 15-conductor, \#18 AWG cable, used for signal transmission, reclaimed from an Ontario-Hydro fossil plant.

- A coaxial cable of the type used in nuclear power plants, obtained from the test program at Sandia.

*AWG: American Wire Gage 
- A general-purpose (RG58) coaxial cable, not qualified for nuclear power-plant service, but tested for comparison with the performance of the nuclear power plant type of coaxial cable.

\subsubsection{Simulation of defects}

Two methods have been used to obtain a cable containing a "defect":

1. The simple approach of damaging a cable specimen that was initially sound is the cleanest, and provides a direct one-to-one, before-and-after comparison. However, it is irreversible and consumes substantial amounts of cable because, to be a realistic test, one specimen requires tens of meters of cable.

2. Another approach is to prepare "standard defects" on a short piece of cable, then insert this defect between two lengths of sound cable. In this approach, the sound cable is not consumed, the process is reversible, and it allows insertion of a variety of standard defects in a sound cable (assuming that the 'sound' cable does not contain defects unknown to the operator).

However, the resulting splices become the site of substantial discharges that can mask the discharge from the defect site. To alleviate the problem of having these substantial discharges drown out those associated with the defect, several attempts were made at reducing the intensity of these discharges by restoring the insulation of the conductors that were stripped for making the splice. Both silicone sealant and silicone putty were used in these attempts, but they were not successful. Another approach was then taken, by immersing the bare splice in $\mathrm{SF}_{6}$ contained in a sealed plastic tubing sleeve (figure 7-3).

As a safety precaution, tubing was provided to inject, then to vent the $\mathrm{SF}_{6}$. This venting arrangement also allowed making a comparison between the discharge pattern in air and in $\mathrm{SF}_{6}$, by alternating the medium around the splice without otherwise disturbing the splice configuration. The comparison clearly indicated the beneficial effect of the $\mathrm{SF}_{6}$ medium. This gas shield technique was also used to connect the cable to the detector in some instances, because that connection appears to be the major source of discharges (a check of the power supply and detector without the cable being connected indicated very little partial discharge activity). The gas experiment was conducted in response to some concern that in a field application, hardware would need to be developed for coupling the in-situ cable to the test gear without creating such spurious discharges.

Several types of artificial defects have been used in the experiments. Cables damaged in service could not be obtained, so that the "defects" used in these experiments should eventually be validated as being representative of "real defects". Using a set of standard artificial defects on short specimens was more effective as it 


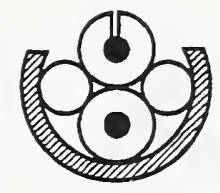

A

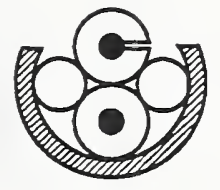

B

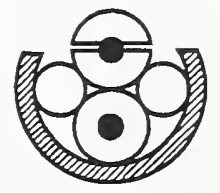

C

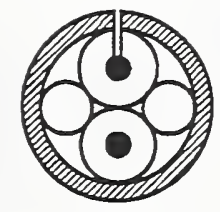

D
A. Single hole, at $0^{\circ}$

B. Single hole, at $90^{\circ}$
C. Double hole, at $90^{\circ}$ and $270^{\circ}$

D. Hole through jacket and conductor

P-D 8-1

Figure 8-1. Artificial defects created in two-conductor cable

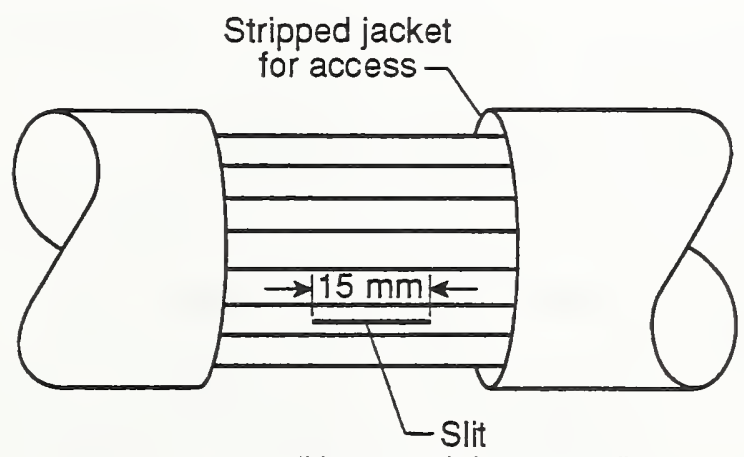

(No material removed)

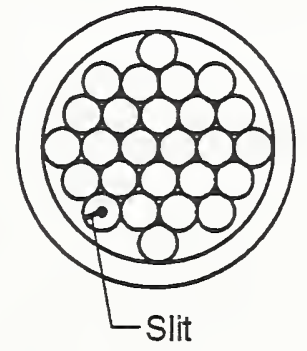

P.D 8.2

Figure 8-2. Artificial defect created in multiconductor cable

allowed replication and comparison of test results. The defects tried on the various cables include those illustrated in figures 8-1,8-2, and 8-3. Other disturbances of the dielectric structure were also created by placing metallic objects near the cable.

Figure 8-1 shows several types of defects created on the two-conductor cable by cutting away the outside jacket and slicing or drilling into the conductor sleeve with a \#60 drill, reaching the conductor. This hole can be drilled in various orientations with respect to the second, undamaged conductor. It is noteworthy that the defect orientation (A) places little dielectric stress on the defect if the voltage is applied with respect to the second conductor. In the tests, that defect could not be detected at the present state of signal processing capability, and it is doubtful that it can be detected at all. Yet, under loss of coolant accident (LOCA) conditions, such a defect could produce significant leakage paths. Other defects were created by drilling two holes, at a distance of $10 \mathrm{~mm}$ along the length of the cable, one in each conductor, at various angular positions.

Figure 8-2 shows the artificial defect created in a multiconductor cable by first removing the jacket over a short portion of the cable, then cutting a slit (without removing any material) in the insulation of a single conductor. In contrast to the drilled hole defect of the two-conductor cable, this defect became readily apparent 


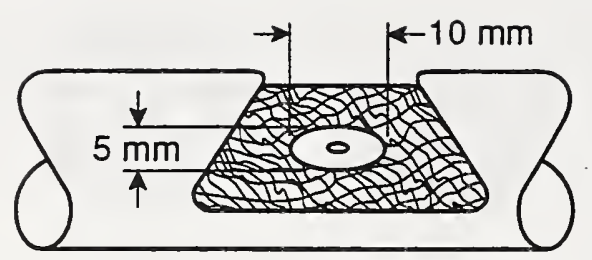

A

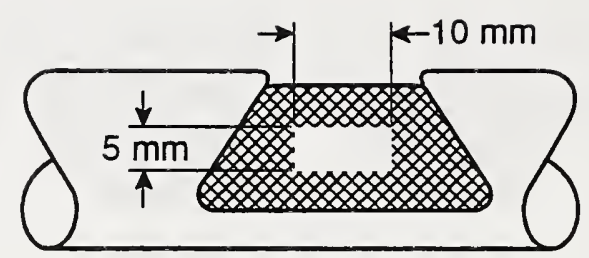

B

A. Window opened into the braided shield (Hole is optional)

B. Window cut into the braided shield

P.D 8-3

Figure 8-3. Artificial defects created in coaxial cable

during the tests, a situation attributable to the multiplicity of grounded conductors near the single conductor being energized.

Figure 8-3 shows the artificial defect created in coaxial cables by prying apart the wires of the braided shield (A) or cutting away part of the braid (B), and eventually drilling a hole into the dielectric, reaching the conductor, should it become necessary to increase the severity of the artificial defect. The nuclear plant grade coaxial cable has a very tight braid that cannot be pried apart, in contrast to commercial grade RG58 coaxial cable, which was not considered representative of nuclear power-plant material. Thus, there was some curiosity as to whether the sharp tips of the cut braid would cause greater discharges than the more gentle prying apart of the RG58 shield. The test results, limited to a few trials, did not show an obvious difference as both defects could readily be found.

While the presence of a metal object adjacent to the cable is not properly a "defect", this presence might produce stress concentrations and thus promote partial discharges that could be interpreted as a defect. The concern here was that an unshielded cable in a cable ladder might be a difficult test case. This concern was in part triggered by anomalies during a series of tests on the two-conductor cable, a large number of background discharges being first attributed to the presence of metal chips picked up when the cable was spooled in a machine shop. A simplified, gross representation of such a situation was established as shown in figure 8-4, with a steel rod tangential to the cable (A), or across the cable (B).

In this context, it should also be noted that loose coils of cable tend to produce noisy traces as the traveling pulses tend to couple across turns. Should the coils be moved, the tests results could be erratic. To avoid that situation in the basic measurement series, the two-conductor cable was wound on a wooden mandrel. The result was a much cleaner pulse pattern. Of course, this simplification would not be available for in-situ tests, so that the matter needs further exploration. 


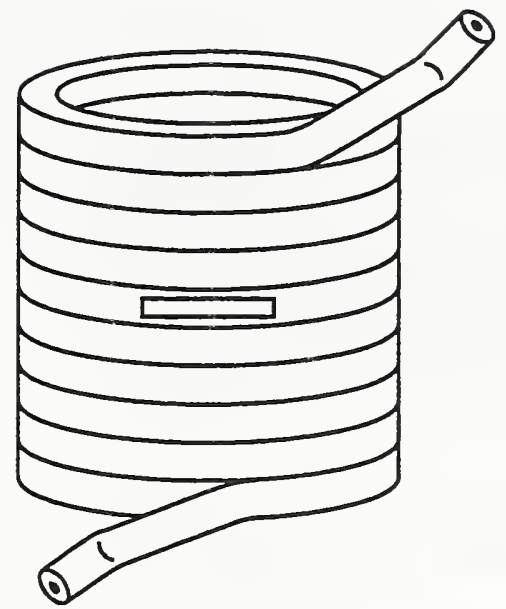

A

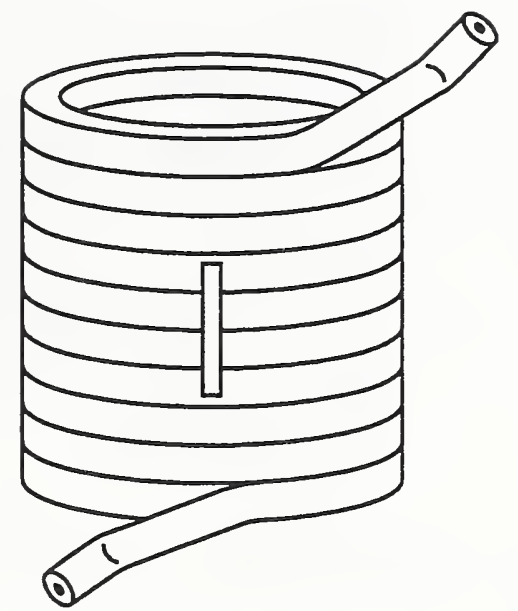

B

A. Adjacent metal object, tangential

B. Adjacent metal object, across conductors

P-D 8-4

Figure 8-4. Adjacent metal objects near cable

\subsection{Measurement results}

This section presents the results obtained on the three types of cables described in Section 8.2, typical of those installed in the low-voltage systems of nuclear power plants. Initial experiments are briefly described to provide a perspective on the final test procedure developed for the menu-driven system described in Section 7. The effectiveness of the test method was demonstrated through a blind test where the existence and location of defects, unknown to the test operator, emerged through a set of measurements performed over a range of applied test voltages and with appropriate pruning of the data.

\subsubsection{Initial experiments}

Some initial experiments were necessary to define the measurement problem. Many questions needed answering with some of the more important questions being:

1) Will the propagation characteristics of the cables limit (or even prevent) the detection of partial discharges?

2) Do defects have partial discharges associated with them?

3) What level of PD sensitivity is necessary for detection of defects? 


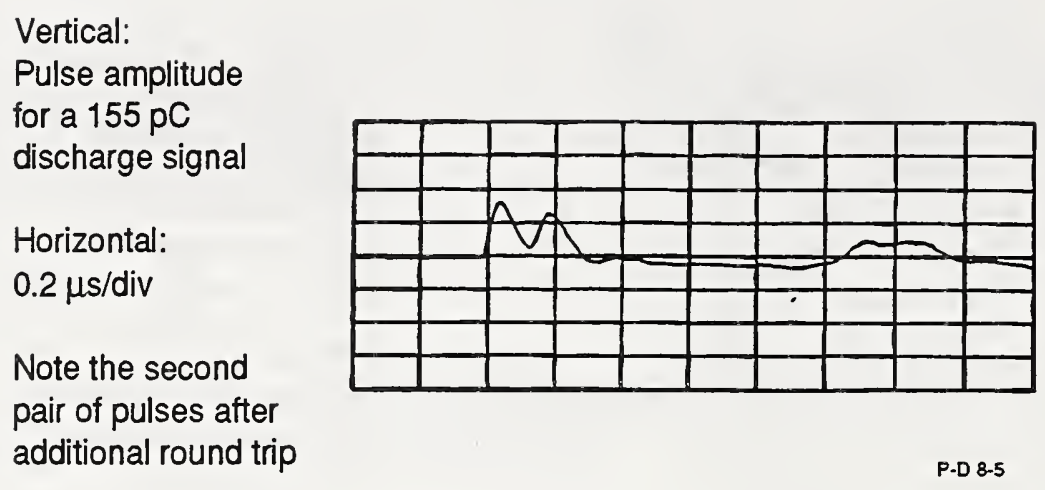

Figure 8-5. Single record of a calibration pulse, direct and indirect arrivals at the detector (near) end of the cable

4) Do other, innocuous, cable anomalies have partial discharges associated with them?

5) Will interference limit the capability for detection of the defects?

6) What is the best way to measure the existence of the defects using partial discharges?

During the initial period when the Asyst-based, menu-driven system was being developed, early experiments were performed using either the acquisition program supplied with the digitizing oscilloscope or with the plotting routines built into the digitizing oscilloscope. These first experiments were conducted on a length of threeconductor, \#12 AWG stranded cable of the type used for heavy duty power cords. This cable was selected because it appeared to be the most difficult of the cases to be considered.

The first question that was answered concerned the propagation characteristics of the cable. This three-conductor type of cable is unshielded and as such is susceptible to interference. Furthermore, being unshielded, external conducting objects in the vicinity will influence any pulses propagating along the cable. This type of cable has also many minor impedance discontinuities distributed along its length since little care is taken in the design and manufacture to make the propagation path uniform. These non-ideal properties make it difficult to measure precisely the propagation characteristics. However, it is not so much the exact characteristics that are of interest here, but rather determining whether a pulse would propagate along the cable. Fortunately, it turns out that pulses do propagate along this type of cable, as can be seen in figure 8-5, and actually maintain a surprising amount of fidelity.

Initial experiments pointed out some problems with the first receiver design. The direct pulse was received clearly but the reflected pulse was severely attenuated and could hardly be observed. The decision was then made to reduce the lower cut-off frequency of the high-voltage receiver network from $850 \mathrm{kHz}$ to $30 \mathrm{kHz}$. Lowering the 


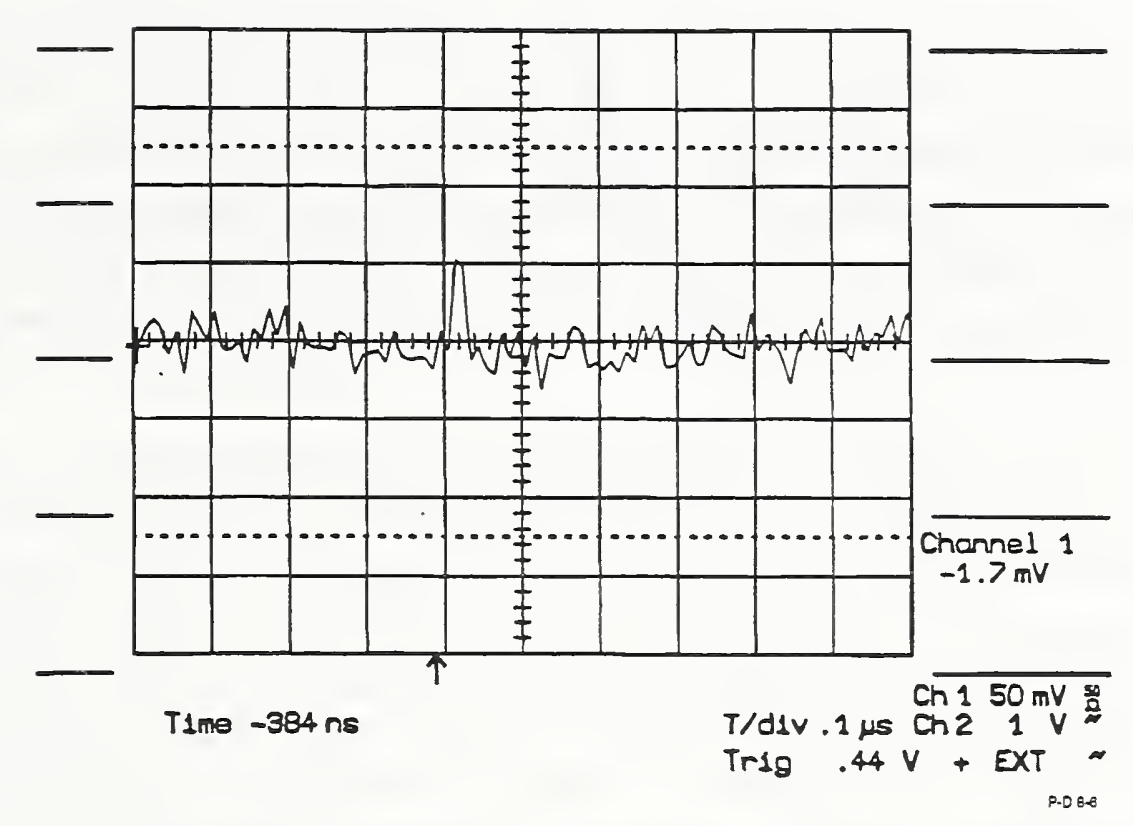

Figure 8-6. Response of measuring system to a $0.01 \mathrm{pC}$ PD calibration pulse

cut-off frequency reduced the attenuation and made both the direct and reflected pulses observable. In the original design, the receiver was matched to the impedance of the cable under test. In the second design, the receiver had a $1 \mathrm{k} \Omega$ input impedance so both the far and near ends of the cable were effectively an open circuit. This opencircuit condition resulted in several reflections for each PD pulse. Figure 8-5 shows some of these secondary reflections with only a moderate amount of attenuation.

The other initial questions were more difficult to answer since they can not be answered separately. Therefore, the approach was to minimize the noise in the test apparatus and then begin testing for the lowest level possible at which partial discharge could be observed. Once again, the worst-case cable was the threeconductor cable because it is unshielded and most susceptible to noise. Careful attention was given to shielding and grounding of the test apparatus and of the measurement equipment to minimize noise. A coaxial geometry for the receiver was developed to minimize noise. Coaxial high-voltage line filters were built to eliminate noise from the power source. Eventually the noise was reduced to a level such that it was possible to detect a $0.01 \mathrm{pC}$ calibration pulse in the test apparatus. A $0.01 \mathrm{pC}$ calibration pulse measured with the test system is shown in figure 8-6. This sensitivity was exclusive of any signal processing used to enhance the capability for detection. This level is much lower than one could expect to achieve in a non-laboratory setting.

Having minimized the noise in the test apparatus, experiments were conducted to determine if partial discharge could be used as an indicator for cable defects. Almost immediately, question four (Do other, innocuous cable anomalies have partial discharges associated with them?) was answered. There are indeed innocuous cable anomalies that have partial discharge associated with them: the ends of the cable exhibited large PD activity. The cable terminations had so much PD activity that 
other "quieter" sites within the cable were completely obscured. A great deal of effort was devoted to finding a "good" solution to this problem. Finally, it was decided that the ends of the cable should be immersed in oil. This method is not a practical solution because of the difficulties of doing this in the field. However, for experimental purposes, it provided a quick solution so that the work could go on toward answering the other questions. A "good" solution is still needed before a practical system can be developed.

Partial discharges at the termination points also limited the number of experiments that could be performed. To be certain that the detected PD pulse is from the intended defect and not from some other source, one needs to be able to determine the origin of the pulse. It is difficult to determine the position of a partial discharge site within a short cable test specimen. The easiest and most reliable method to ensure detection of a known defect is to embed the defect within a long piece of cable. To implement this plan, it was decided to insert a short piece containing defects at a known location along a cable by splicing the test piece between two lengths of sound cable. Unfortunately, the splices themselves became sources of partial discharge and it was impossible to determine whether the partial discharge came from the splices or from the defect placed near the splices. At that time, oil had not yet been considered as a solution to this problem. During the initial testing phase, a fortuitous event took place. Two lengths of cable had been spliced together and the PD activity at the splice was under investigation. For no apparent reason, the partial discharges were especially strong at the splice. The test voltage was applied to the spliced region for an accumulation of many hours while this phenomenon was being investigated. The spliced region failed during this investigation. Upon close examination of the failed portion, it was noticed that this cable had embrittled and, after mechanical stress, had developed a number of cracks in the insulation. This observation was the first indication that $\mathrm{PD}$ testing was capable of detecting a cable defect.

Also, during the initial testing phase, experience showed that the defects did not have a lower inception voltage than the rest of the cable. Initially, a lower inception was expected and assumed; had this assumption been true, then detection of the defects would have been easy. One would simply have to apply a (relatively low) voltage and any observed partial discharge would be from defects. This simple situation definitely is not the case and consequently the detection of defects is compounded. On the brighter side, experience has also shown that defects have significantly different statistical behavior than the other partial discharges in the cable. Defect sites seem to have larger amplitudes and higher count rates than PD sites not associated with a defect. One interesting question that remains to be answered is whether other statistical descriptors can be used to identify defects. For example, it is quite possible that the correlation (memory) between pulse amplitudes and times is markedly different for defects and non-defects. If this were the case, then it would be a much simpler task to automate an analysis program. 


\subsubsection{Preprocessing}

It certainly is preferable to collect all detectable pulses; however, since the measurement system is a general-purpose type, trade-offs have been made. In this case, generality of the instrument was traded for cost, speed, and data-handling capabilities. With these constraints, only a small number (a few thousand) of pulses can be collected and processed.

When the voltage is raised to the inception level, a large number of low-level background PD pulses appear. Mixed in with this low-level activity, there are larger PD pulses from the defects. Experience has shown that the count rate of the smaller, innocuous pulses is much larger than the count rate of the larger pulses associated with the defects. Using the general purpose equipment to collect all partial discharge pulses regardless of there origin results in a data set that contains few if any pulses from the defects. The number of PD pulses from defects detected in this manner is so small that the deviation in the delay histogram is on the order of the variance of the histogram. In other words, the deviations due to defect sites at those positions within the histogram are statistically insignificant and therefore the defect site is not readily detectable.

The best way to collect data is to use a system with a large dynamic range and a large memory. Using such a system allows collection of all of the pulses, even the smallest and the largest. The advantage of collecting a large number of PD pulses is that the variance of the histograms becomes smaller as the number of pulses is increased. Existing measurement systems (Pulse Height Analyzer) based on this fact are routinely used to collect PD data but are not capable of determining the location of the PD site. Even if the measurement system could process large amounts of data, it is still likely that defects could become obscured when the background partial discharge is intense.

One method that has proven useful to improve the detection is discrimination. Discrimination is a method in which limits are placed on a parameter of the data to qualify it as valid. The simplest parameter to use is the amplitude. In this case, only PD pulses with a large enough amplitude are considered valid. This method is well-suited for use with the digitizing oscilloscope because a trigger threshold (discrimination level) must be selected to make it work. So not only is it desirable, but also it is necessary to select a discrimination level.

At this point, there is no clear justification for choosing any particular threshold. Experience with this technique has shown that raising the discrimination level results in improved defect detection. The reason for this behavior is that PDs from defects observed so far have always had larger amplitudes than the background level. On the other hand, setting a low threshold clutters the data-set with events from innocuous sites. The histogram estimates are formed using only a few thousand data points. So even moderate amounts of clutter from the background partial discharge is enough 
to obscure weak defect sites. There is a drawback to using a high threshold: it slows down the rate of acquisitions. While there is no assurance at this point that only the largest signals are necessarily those associated with the defects being sought, so far it has yielded positive results.

The selection of the trigger threshold has considerable influence on the outcome of the test. As discussed in Sections 5 and 6, one could expect a relationship between the amplitudes of the discharges and the nature of the sites (defects) giving rise to the partial discharges. However, at the time these experiments were conducted, this relationship.was not established for the artificial defects inflicted on the specimen cables. It was mentioned that discrimination can be used on any parameter. One could use statistical parameters such as correlation with previous events to discriminate data. As discussed in Section 10, research in this area may be a fertile ground for improvements. Another approach that could be used relies on a Pulse Height Analyzer. If the measurement system included a Pulse Height Analyzer, regions of interest could be selected, based on its measurements, and only pulses from the regions of interest would be processed further.

\subsubsection{Pulse height analysis}

Each PD pulse is collected as a waveform. After the raw data are collected, each waveform is reduced into two data points: an amplitude and a position. The amplitude portion of the reduced data can be presented in tabular form or as a histogram of number of pulses versus charge. Figure 8-7 is an example of this output, with the vertical axis showing the number of counts and the horizontal axis the value of the discharge. For this plot, the data have been separated into positive and negative discharges. The tabulation yields precise information on the distribution, while the histogram conveys a message of "profile", a useful tool for further analysis.

As discussed in Section 6, the profile of such a histogram in a cable free from preferred discharge sites can be expected to be different from that of cables containing preferred sites - presumably a defect. In a cable free from defects, one would expect that all partial discharge sites would be similar. Innocuous PD sites in this case are expected to arise from interfacial partial discharge caused by air gaps between the conductors and the insulation used to cover them or by the air gaps between the insulation covering the separate conductors, as in a multiconductor cable. In cables of the type of interest, one would expect a large number of innocuous sites because the manufacturers do not intend for these types of cable to be operated at the high level of stress being considered here. If this is the case, then it is expected that the resulting histogram of amplitudes are a superposition of similar histograms associated with lower PD levels that could mask the presence of a defect. This type of behavior is illustrated in figure 8-8. 


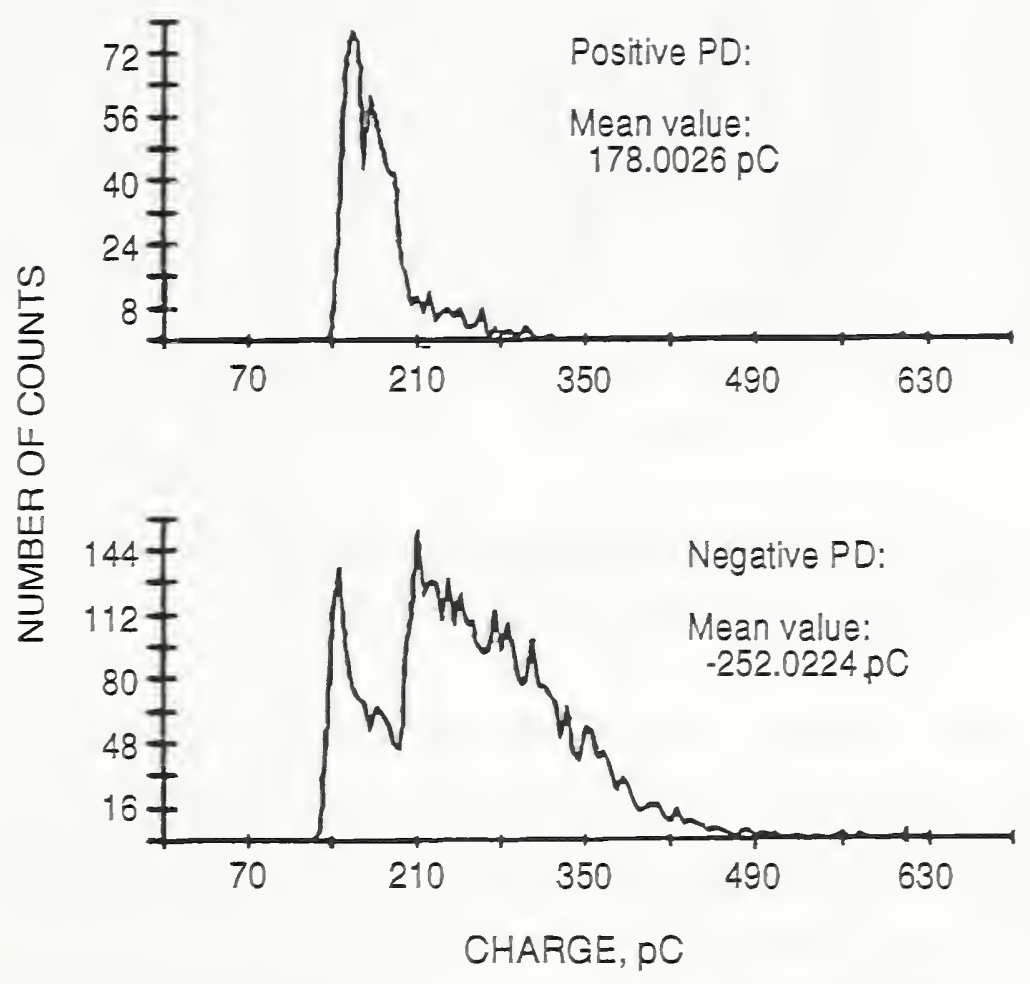

Figure 8-7. Histogram of the distribution of PD pulses as a function of their charge transfer magnitude ("pulse height")

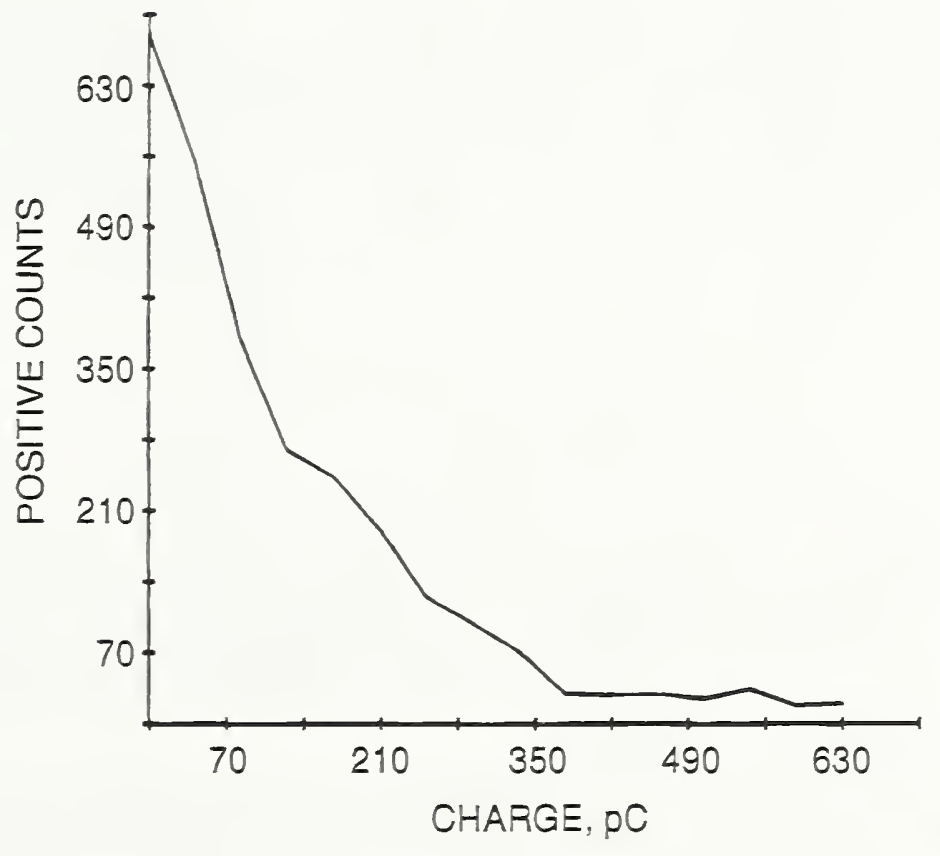

P.D 8-8

Figure 8-8. Typical pulse height distribution for multiple low-level innocuous partial discharges 


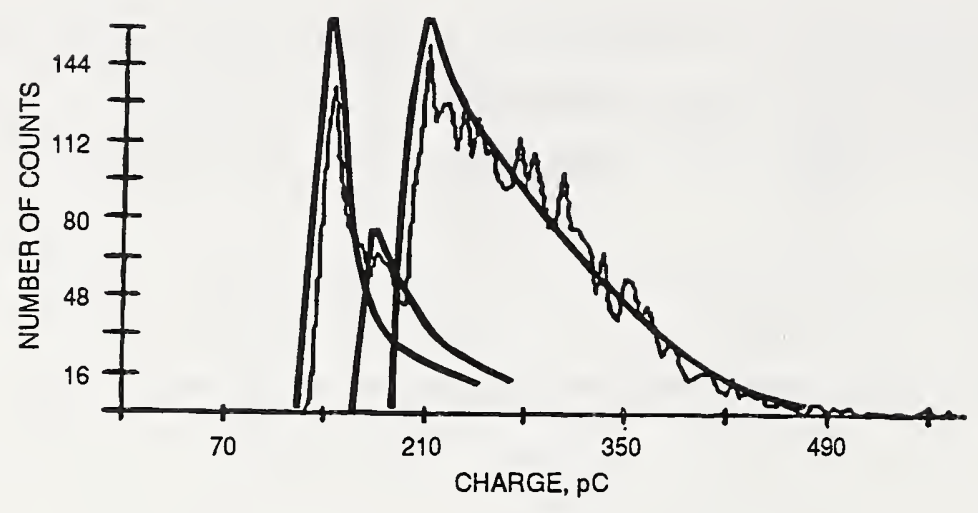

P-D 8-9

Figure 8-9. Histogram of negative partial discharges from figure 8-7, with interpretation of the multiple pulses as the sum of three separate distributions

In contrast to the normal condition, defects are presumed to arise from complete exposure of the conductor through a large air gap (for example, through a crack). In this case, the histogram still superposes with the other innocuous sites but is shifted out to higher PD levels. These shifted histograms appear as irregularities in the histograms. Detecting such anomalies can serve as the basis for concentrating further analysis on that part of the histogram, to enhance the likelihood of isolating the preferred site from the background. In figure 8-7, both histograms obtained from a two-conductor cable specimen show a double-peak pattern, which can be considered to be the superposition of two separate distributions. This interpretation is illustrated in figure $8-9$ by the solid lines manually added to the plot obtained.

Only a small amount of data is collected during any one trial, so one might consider it superfluous to restrict further the data by additional discrimination (recall that one level of discrimination, threshold, has already been applied). However, further restriction by concentrating on the anomalies does serve the purpose of demonstrating the fruitfulness of the procedure. When compared with the resulting delay histograms, the method is vindicated because the result is a single delay peak. This selection criterion is still an intuitive matter, but it may be amenable to automated processing when combined with a Pulse Height Analyzer. The method takes advantage of the increased PD levels associated with defects. Should the defects behave like the rest of the innocuous partial discharges then the method would fail. If the cable has a large number of defects, then it is expected that anomalous regions do not exist because the resulting histograms are a superposition of a large number of defects. In this case, the defects would resemble innocuous background partial discharges but with a high mean amplitude. 


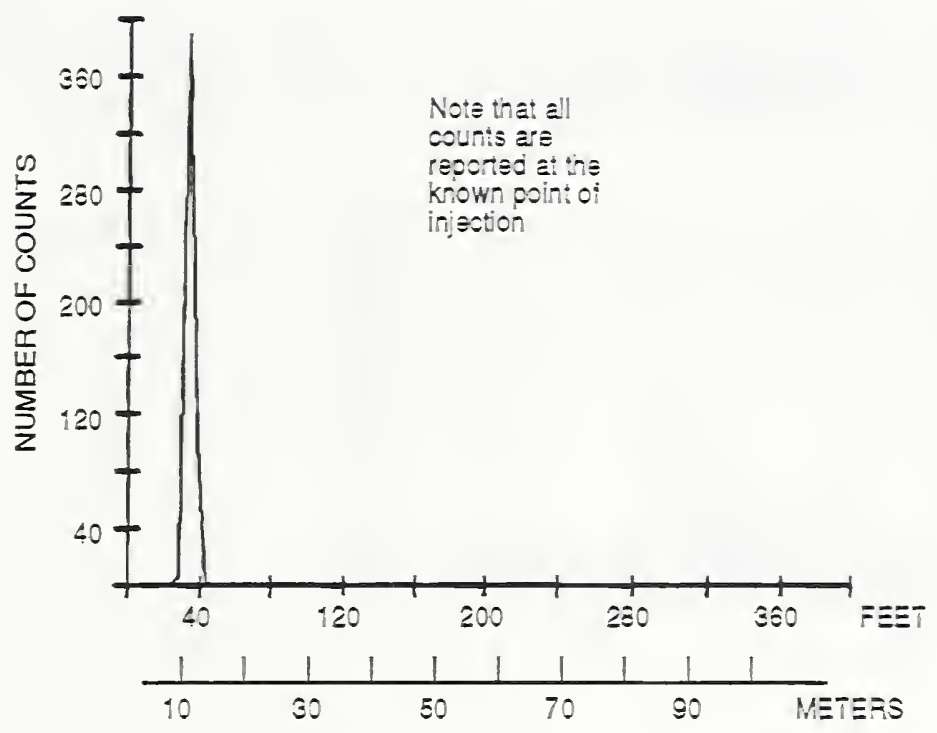

$P-D \leqslant-10$

Figure 8-10. Histogram of time delays, converted into feet from the open end, for 500 calibrated pulses injected at $12 \mathrm{~m}$ ( $35 \mathrm{ft}$ ) from the far end of the two-conductor test cable

\subsubsection{Delay analysis}

It was mentioned that each PD waveform is reduced to two data points. The position of the PD pulse is calculated from the delay as discussed in section 6. The delay analysis method is based on the hypothesis that the two major peaks recorded in any one event ("element") are in fact the two pulses (direct and reflected) arriving from the same site. Thus, the Asyst software processes the data for each erent by searching for the two absolute maxima in each single-event record. Actually; a fraction of all events in any sequence may contain a pair of two major but unrelated pulses originating from separate partial discharges. The software does not make a discrimination and will interpret these other pairs as coming from the same site and show the results in the data-reduction output. However, these unrelated pairs occur at random and have no consistent correlation, while the paired pulses truly coming from the same site will yield a fixed location in the counting process. The probability of this random occurrence is small at the roltage levels used. The separation between pulses of this type is random and thus will not lead to a count rate that favors any particular site. On the other hand, the histogram showing the difference of time between the arrivals of paired pulses will exhibit a greater number of pairs at the fixed sites.

To illustrate this point, return to figure $8-5$ which shows a simple example of a single waveform of a pulse being injected by a calibrator circuit at an artificial defect created in the test cable at $12 \mathrm{~m}$ ( $35 \mathrm{ft})$ from the open end. In this manner, the defect site is the only source of paired pulses. With such a clean data base, the delay analysis, predictably; shows all the emanation points at a single site. Figure 8-10 shows the 


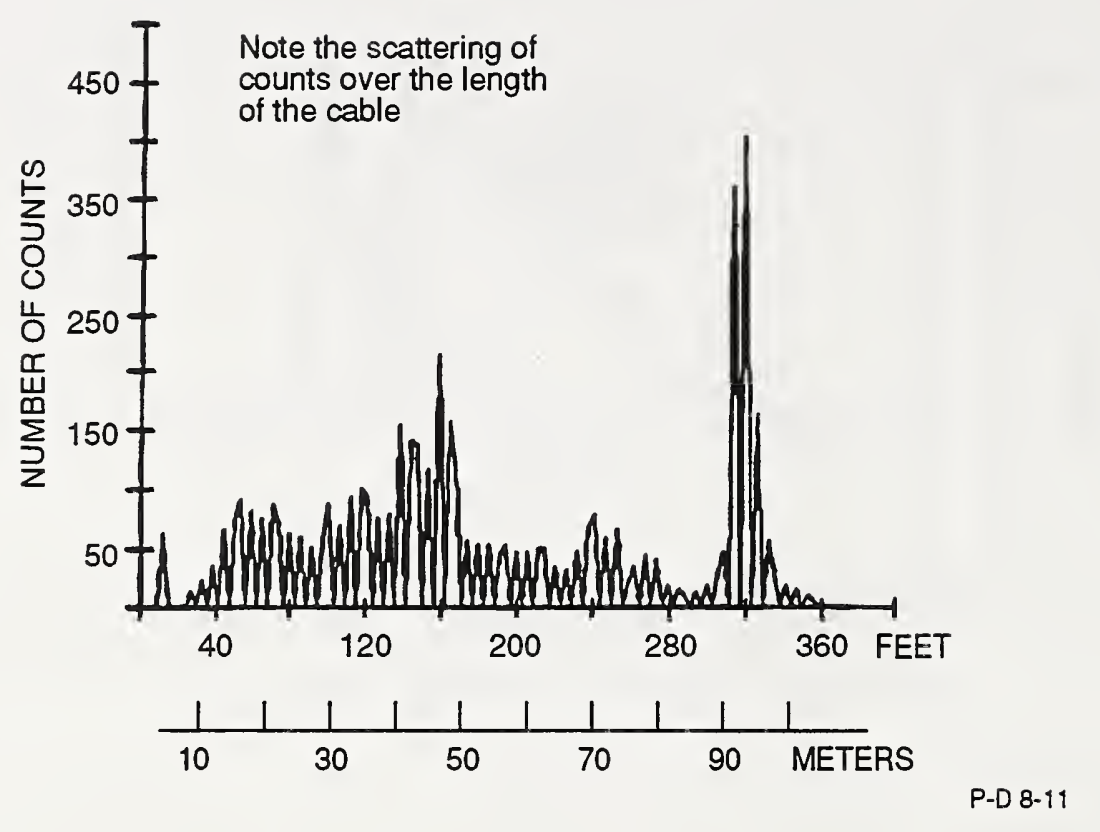

Figure 8-11. Histogram of delay times between the two major pulses in each event of a 5000 -element record containing true pairs and random pairs

histogram generated for 2 records of calibrator pulses (total of 500 elements). The number of discharges is shown on the vertical scale; the distance from the open end of the cable is shown on the horizontal scale. The histogram has a triangular shape because the bin width selected for sorting process allocated counts in more than one bin.

In the data that follow, there appears to be an inconsistency in reported locations. The variations are slight and are caused by the manner in which the histogram bins are allocated by the software. The total histogram length and number of bins used in the histogram determine the boundaries of each bin. As a consequence, delays falling within the boundaries of any one bin are reported to have delays equal to the center of the bin. The important information relayed by the histogram is that the partial discharge is in the interior of the cable and not at the end.

If the discrimination level is set low enough to the point that the innocuous background partial discharges dominate the count rate, then the delay histogram will not show preferred sites. In the ideal case, where all the background sites are statistically similar, the delay histogram will correspond to a uniform distribution, that is, the sites are uniformly spread along the length of the cable. Figure 8-11 shows an example of this situation, which at first appears hopeless. However, the techniques of applying different levels of voltage stress, of selecting "suitable" threshold levels during the data acquisition, and of pruning the data from the pulse-height analysis, all contributed to the successful demonstration described later in this report.

To illustrate the effectiveness of discrimination as a preprocessing step, the analysis program incorporates a procedure to select a region of interest from the pulse-height 
histogram. The resulting delay histogram, in the experiments performed, always showed that the anomalous regions of the pulse-height distribution correspond to a preferred site.

To perform this selective delay analysis, the screen cursors described in Section 7 were used to select a portion of the histogram and discard the data points outside of the selected area. Not only does this selection speed up the process of data reduction, but it promotes the emergence of distinctly abnormal points in the histogram of delays. At this point, however, the selection of which part of the histogram should be analyzed remains a matter of intuition. As discussed in Section 10, statistical analysis methods may be developed to implement an automated, expert-system. This development would require a substantial effort from signal processing specialists, an effort which was not within the scope of the project, that is, the assessment of the capability of known test methods.

\subsubsection{Experimental procedure}

The initial experiments discussed in the preceding paragraphs helped define an approach. Many initial trials not described in this report aided in the development of software programs used to acquire and analyze the data. The systematic experimental procedure that was finally developed is the following:

- The specimen was prepared for the test. The length was measured as precisely as possible. All foreign particles were removed from the jacket of the cable under test. The cable was fastened to a wooden fixture (only the two-conductor cable required this step). The ends of the cable were prepared. The jacket was stripped back at least three inches and the conductor to be tested was physically separated form the other(s) to provide high-voltage isolation. At least one end of the cable was immersed in oil.

- The measurement system was calibrated. A 150 pC calibration pulse was injected at the near end using a broadband calibrator. The propagation delay of the cable was determined using the measurement system. From this time-delay calibration, a velocity was calculated. The known PD pulse magnitude provided an amplitude calibration. Attenuators were used to reduce the magnitude of the PD pulses to maintain them within the range of the oscilloscope display. The attenuators were accurate to $1 \mathrm{~dB}$, so that calibration at a single level was deemed adequate.

- Voltage was applied to the specimen until some low-level PD pulses were observed. This voltage level was called the PD inception voltage and corresponded to receiving at least two PD pulses per second. 
- The test voltage was raised a predetermined percentage above the inception voltage. The discrimination level (trigger level) was set to the appropriate level for the test.

- Data were collected and archived on diskettes for further analysis. The data acquired for signal analysis consist of several records. In this context, "record" is defined as several successive discharges as recorded by the digitizing oscilloscope operating in the sequential mode. In the test procedure implemented by the menu-driven process used in these tests, one record consists of 250 individual pulses, recorded whenever their amplitude exceeds a preset threshold. Typically, a test consists of collecting 20 records (5000 individual pulses).

- The data were analyzed using the computer programs developed for this purpose.

\subsubsection{Coaxial cable experiments}

The coaxial cable is the simplest of the cable types to be considered. The propagation characteristics are well defined, as is the stress placed on the dielectric material. The test procedure was applied to two different coaxial cables. During the initial tests, a length of RG-58 cable was used as an example of coaxial cable. Later on, a coaxial cable of the type used in nuclear power plants was obtained from the test program at Sandia. The major observable difference between the two types of cable was the shield configuration. The shield of the nuclear cable appeared to be a more complete (tighter weave braid) and adhered to the surface of the dielectric more tightly. The defect inflicted on the coaxial cables consisted in first removing part of the cable jacket, then cutting the individual wires of the braided shield, that is, the case (B) of figure 8-3. There was concern about the sharp points of the braid cut, but even the RG-58 cable with a window produced by prying the wires apart readily appeared as a defect, and no difference was observed with a window made by cutting the braid.

During the test, when the specimen was raised to the PD inception voltage, the defect site was immediately recognized from the patterns on the digitizing oscilloscope. Partial discharge at the termination points of the cable did not interfere with these experiments. Computerized analysis of data from this specimen immediately revealed the presence of the defect, as shown in figure 8-12. This particular specimen exhibits the attributes of the idealized statistical model previously discussed. Careful examination of the delay histogram reveals partial discharges distributed, in a somewhat uniform manner, along the entire length of the cable. The other aspect of the behavior is the large difference in statistical behavior between the defect and the background discharges; the count rate at the defect is orders of magnitude larger. 


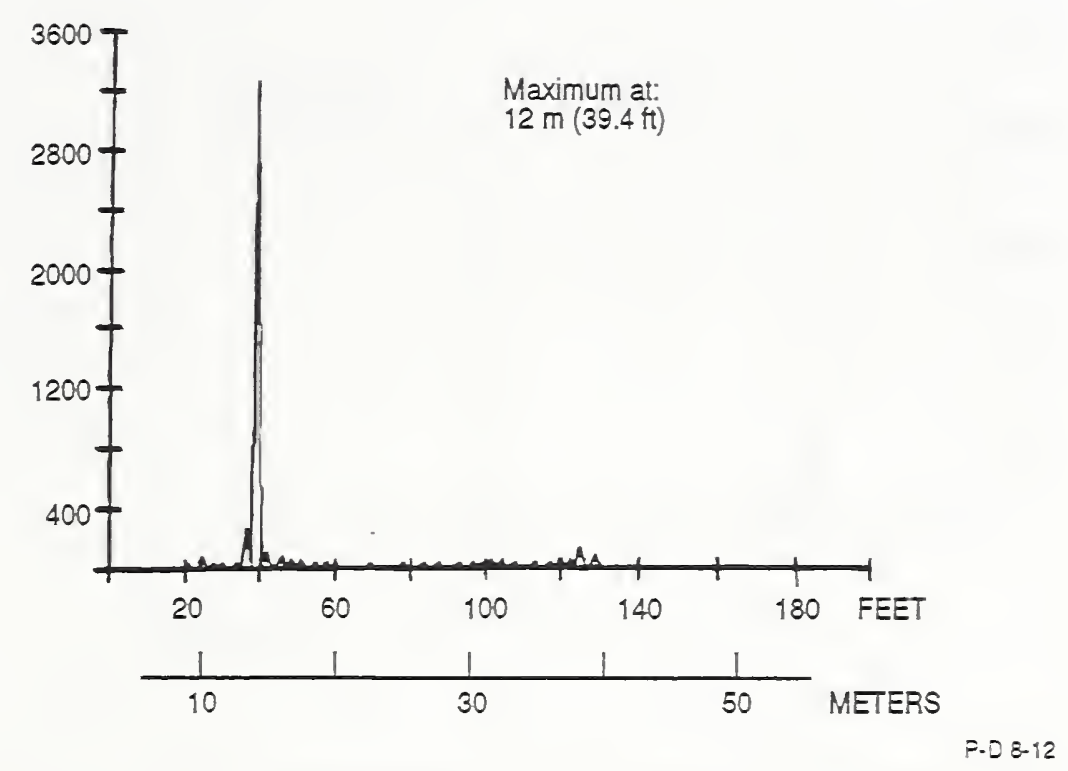

Figure 8-12. Histogram of time delays in nuclear-rated coaxial cable

\subsubsection{Multiconductor cable experiments}

This cable specimen was a 15-conductor, \#18AWG cable, used for signal transmission, reclaimed from an Ontario-Hydro fossil plant. The defect inflicted on this cable consisted in first removing part of the cable jacket, then cutting a slit in the insulation of only one of the wires, as shown in figure 8-2.

The test voltage was applied between that conductor and all the others connected in parallel. The analysis on this specimen immediately revealed the presence and location of the defect, as shown in figure 8-13. The dimension of the damaged conductor is small compared with the remaining conductors, so in effect the damaged conductor would behave in a manner similar to that of a single wire abore a ground plane. Had the damaged conductor been buried within the bundle of grounded conductors, it would have behaved in a manner similar to that of a coaxial configuration. It appears that a large number of grounded conductors in close proximity will make defects simpler to identify.

\subsubsection{Two-conductor cable}

This cable specimen was a two-conductor, \#14AWG, 600-V class, neoprene-jacketed, unshielded cable of the type used by Georgia Power Co. in their nuclear power plants. This type of cable turned out to be the most difficult to test. A total of 30 tests were conducted (each containing 5000 elements), for the various defect orientations shown in figure 8-1, before a clear pattern began to emerge. The breakthrough occurred after recognizing the influence of the test voltage on the pattern of PD pulses. 


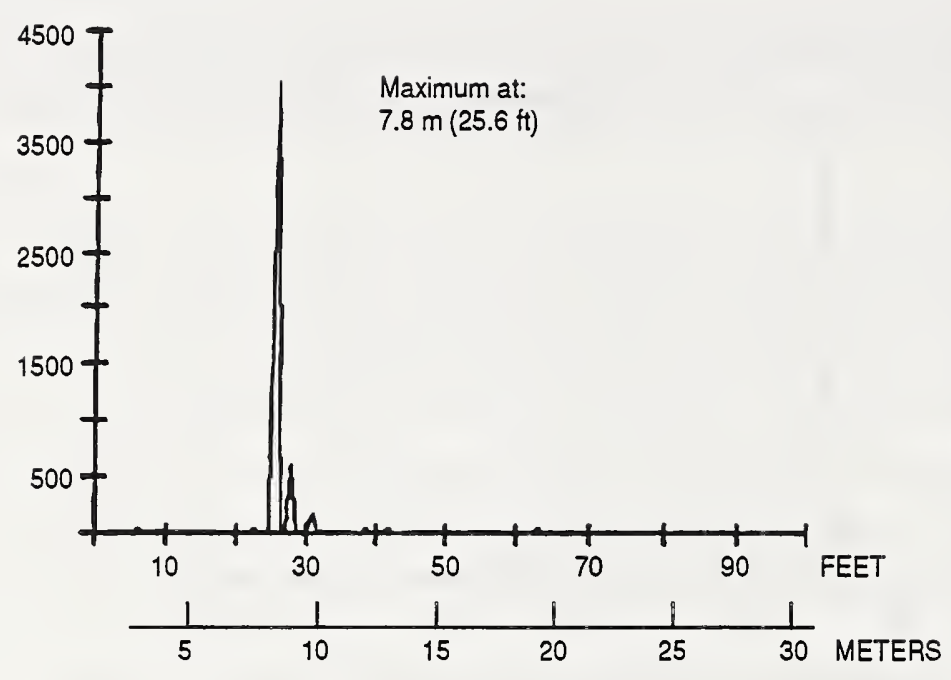

P-D 8-13

Figure 8-13. Histogram of time delays in a multiconductor cable with single defect

\subsubsection{Effect of test voltage}

Only two parameters of the partial discharges (amplitude and position) had been used in the analysis of the preceding experiments. There are a number of other parameters that could be useful. In this particular case, the inception voltage of the individual sites provided further insight. To understand why this makes a difference, the point process model needs to be extended. Only the simplest, time-invariant Poisson model has been proposed as a description of the partial discharge. This model is not very good if one is interested in the fine details of the PD process. However, this simple model does provide intuitive insight into the behavior of the process and how to deal with the multiple sites.

To include the inception voltage into the model for the intensity parameter, $\lambda_{i}$, at the $i^{\text {th }}$ site, $\lambda_{i}$ needs to be modified by including an inception parameter

$$
\lambda_{i}\left(V_{i}\right)=\frac{1}{2} \lambda_{i}\left[1+\operatorname{sgn}\left(V-V_{i}\right)\right]
$$

where $V$ is the applied voltage and $\mathrm{V}_{i}$ is the $\mathrm{PD}$ inception voltage of the $\mathrm{i}_{t h}$ site. The function, $\operatorname{sgn}()$, is the signum function defined as +1 for positive arguments, 0 for a zero argument, and -1 for negative arguments. The result is an intensity parameter that turns on at the inception voltage.

To use this new set of parameters, the test voltage is systematically increased and measurements are made at each new voltage. The change at each step is then observed. As the inception voltage of each site is exceeded, the partial discharge "turns on." This improved procedure uses a systematic application of increasing voltage steps and proper selection of the discrimination level, complemented by selective pruning of the data. 


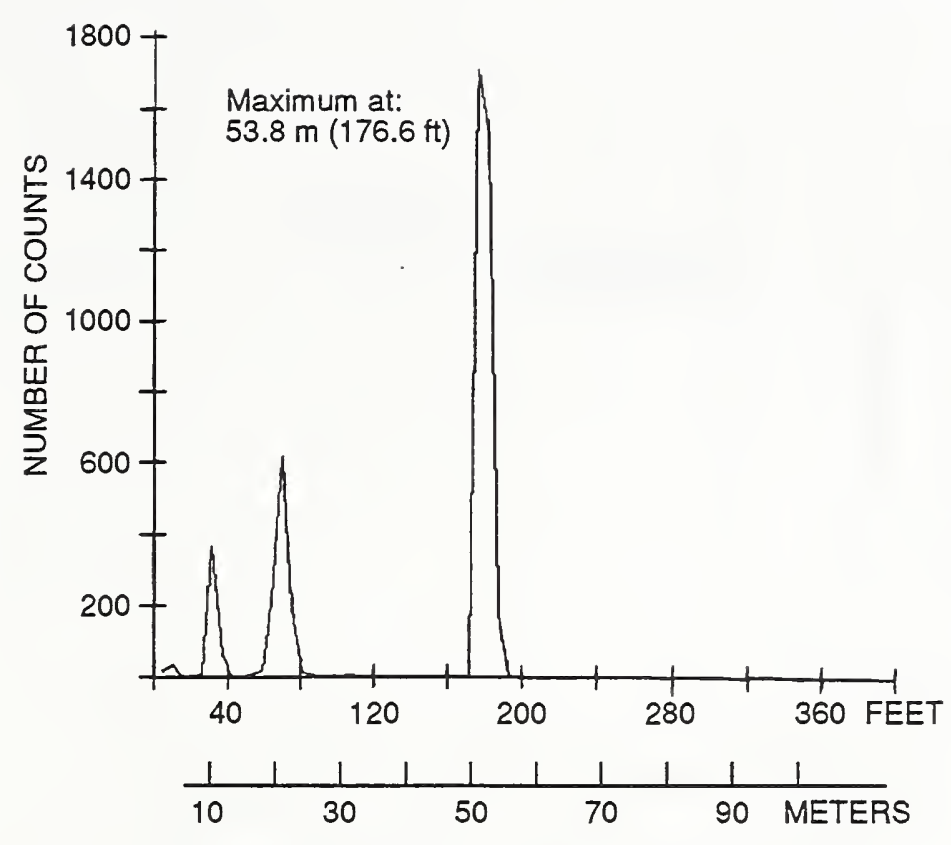

P-D 8-14

Figure 8-14. Histogram of time delays in the two-conductor cable with three defects for $5 \mathrm{kV}$ applied voltage

The additional procedure resulted in a sequence of plots. This sequence is shown in figures 8-14 through 8-19. As can be seen in this sequence, the PD sites suddenly "turn on" when the test conditions are changed. When they turn on, they are fairly intense and in some cases obscure the presence of the other sites. The reason that they obscure the other sites is, that once they "turn on," they have higher count rates than those that are already on.

Table 8-1 summarizes the emergence of the several defects for each of the voltage levels. The additional procedure resulted in a sequence of plots. This sequence is shown in figures 8-14 through 8-19. The histograms of figure 8-14 through 8-19 display peaks covering several bins, so that the total number of counts covers a range on both sides of the maximum distance plotted out on each histogram by the software program. An arbitrary scale of 1 to 100 covering the vertical axis, rather than the number of counts, was used to rank the magnitude of all the peaks appearing at the various distances, a 100 being the highest peak for each histogram. Note how the relative magnitudes among the locations vary from one voltage to another. Some locations are present in all records, while other locations are revealed in only a few. 


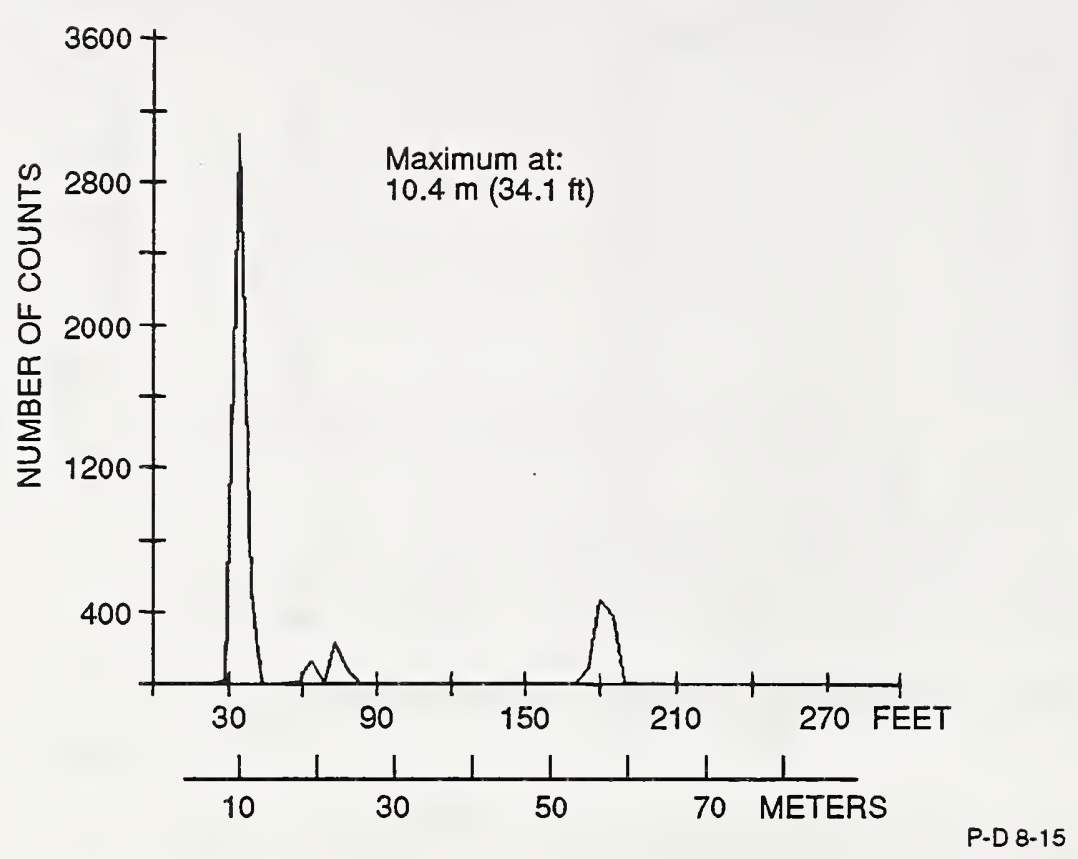

Figure 8-15. Histogram of time delays in the two-conductor cable for $5.2 \mathrm{kV}$ applied voltage

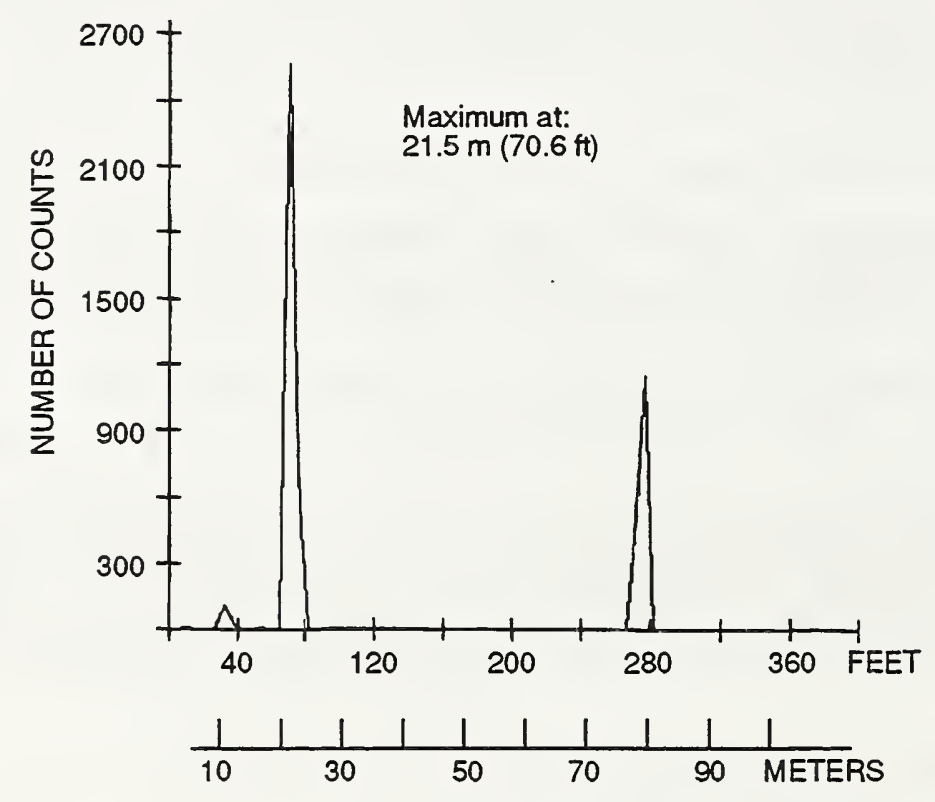

P-D 8-16

Figure 8-16. Histogram of time delays in the two-conductor cable for $5.5 \mathrm{kV}$ applied voltage 


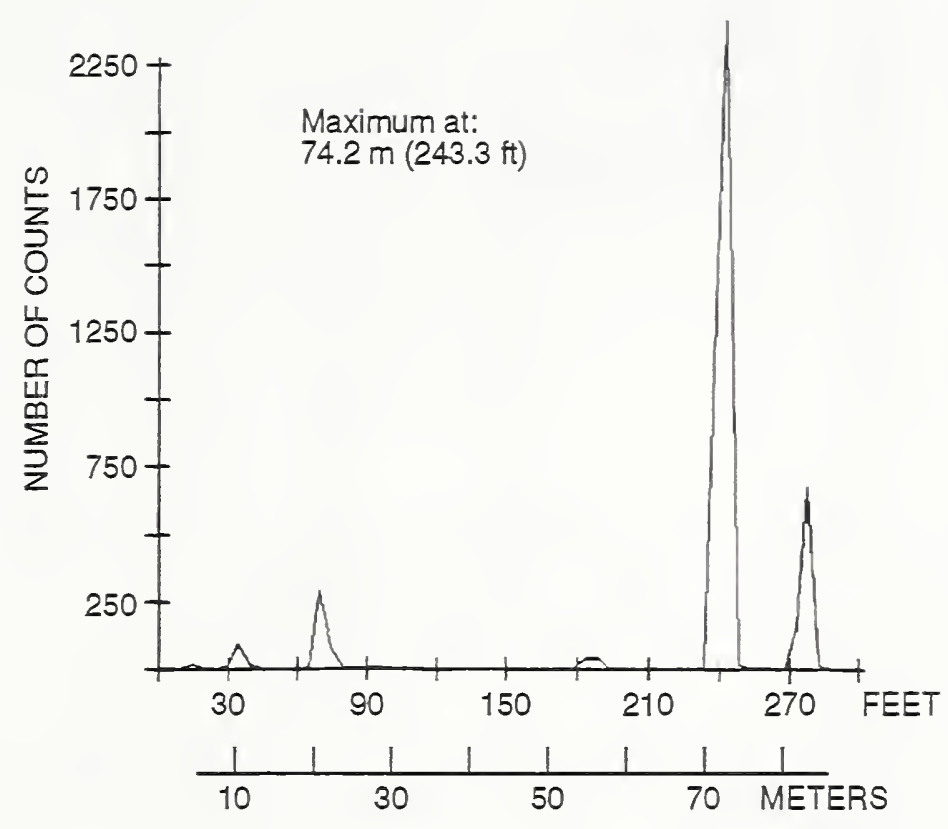

P.0 $8-17$

Figure 8-17. Histogram of time delays in the two-conductor cable for $6 \mathrm{kV}$ applied roltage

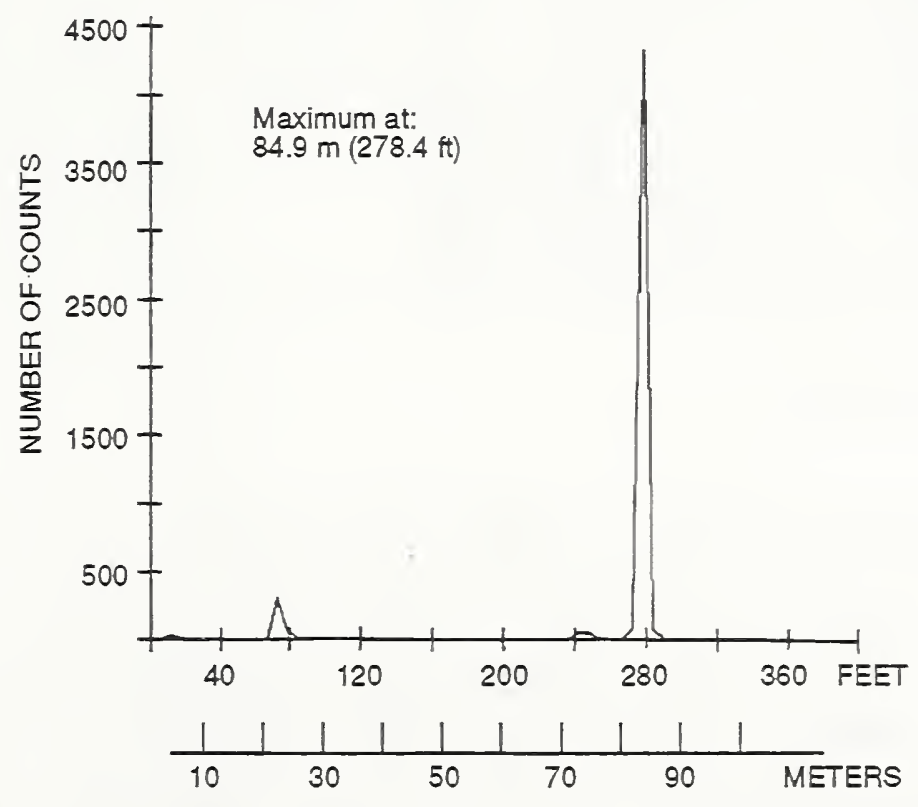

P.D 8-18

Figure 8-18. Histogram of time delays in the two-conductor cable for $6.5 \mathrm{kV}$ applied voltage 


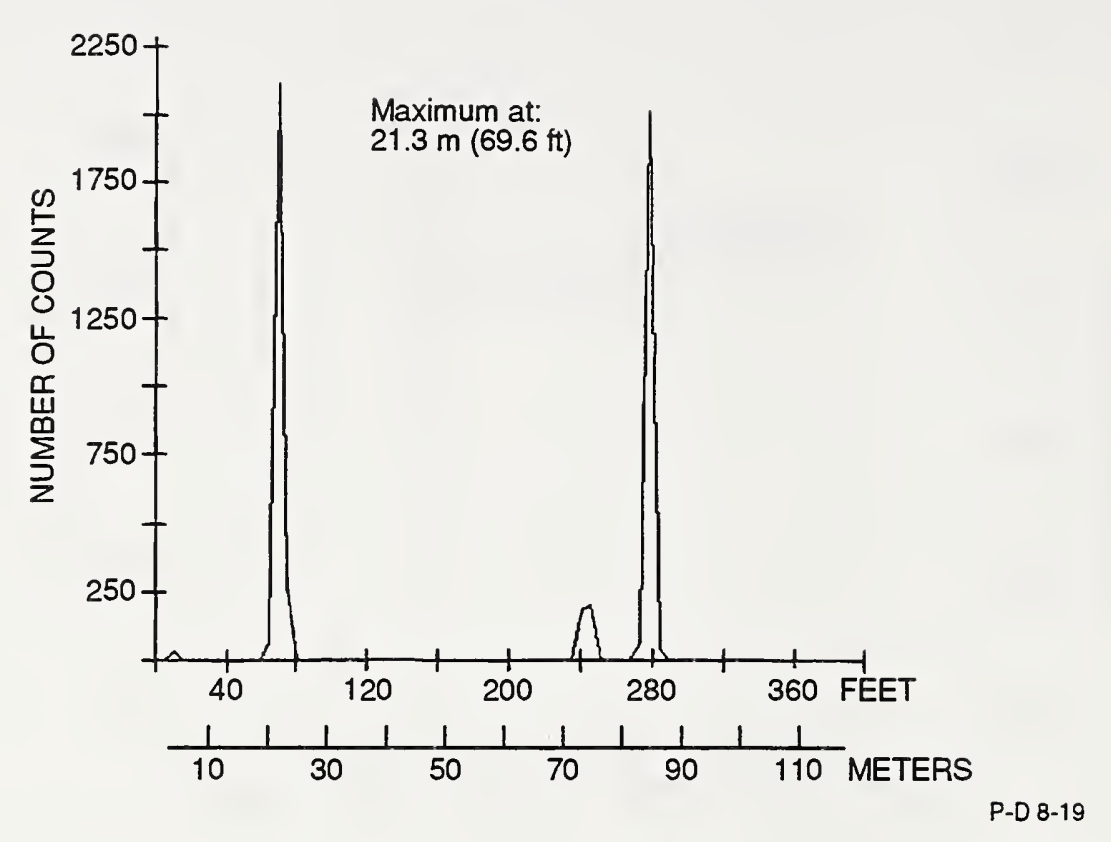

Figure 8-19. Histogram of time delays in the two-conductor cable for $7 \mathrm{kV}$ applied voltage

Table 1. Relative magnitude of emerging defects

\begin{tabular}{|c|c|c|c|c|c|c|}
\hline $\begin{array}{c}\text { Distance from } \\
\text { far end of cable }\end{array}$ & $\begin{array}{l}\text { meters } \\
\text { feet }\end{array}$ & $\begin{array}{r}9.6 \\
32\end{array}$ & $\begin{array}{l}21 \\
70\end{array}$ & $\begin{array}{r}54 \\
180\end{array}$ & $\begin{array}{r}72 \\
240\end{array}$ & $\begin{array}{r}84 \\
280\end{array}$ \\
\hline \multicolumn{2}{|c|}{ Test Voltage $(\mathrm{kV})$} & \multicolumn{5}{|c|}{ relative magnitude (highest $=100$ ) } \\
\hline 5.0 & & 22 & 45 & 100 & & \\
\hline 5.2 & & 100 & 8 & 17 & & \\
\hline 5.5 & & 5 & 100 & 2 & & 45 \\
\hline 6.0 & & 4 & 12 & 3 & 100 & 32 \\
\hline 6.5 & & 2 & 8 & & 3 & 100 \\
\hline 7.0 & & 2 & 100 & & 10 & 95 \\
\hline
\end{tabular}

\subsubsection{Final blind test}

From the totally useless record shown in figure 8-11, progress was made through many additional tests that provided more information and insight on refining the test procedure, such as the effect of the test voltage as well as pruning data before performing the analysis. This progress culminated in a blind test where the operator, applying his educated intuition, correctly identified the location of three defects inflicted on a new length of presumably sound cable, respectively at $10.5,21$, and 72 meters $(35,70$, and 240 feet) from the far end of a 100-meter (333-feet) long cable. Figures 8-20, 8-21, and 8-22 show three histograms from the blind test series on that cable, for different test voltages and a second level of discrimination based on apparent anomalies in the pulse height analysis histograms. This series ultimately revealed all defects, but with different relative distribution of the counts among the 


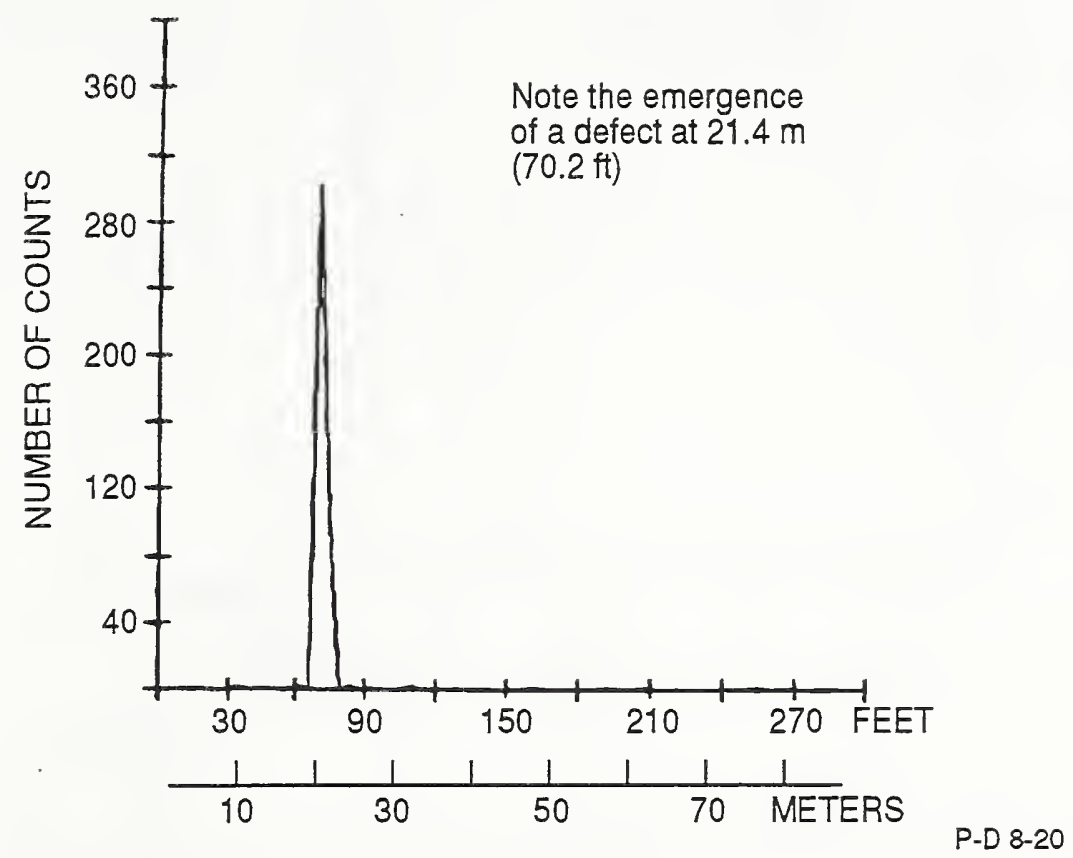

Figure 8-20. Histogram of time delays at $5 \mathrm{kV}$ and pruned data

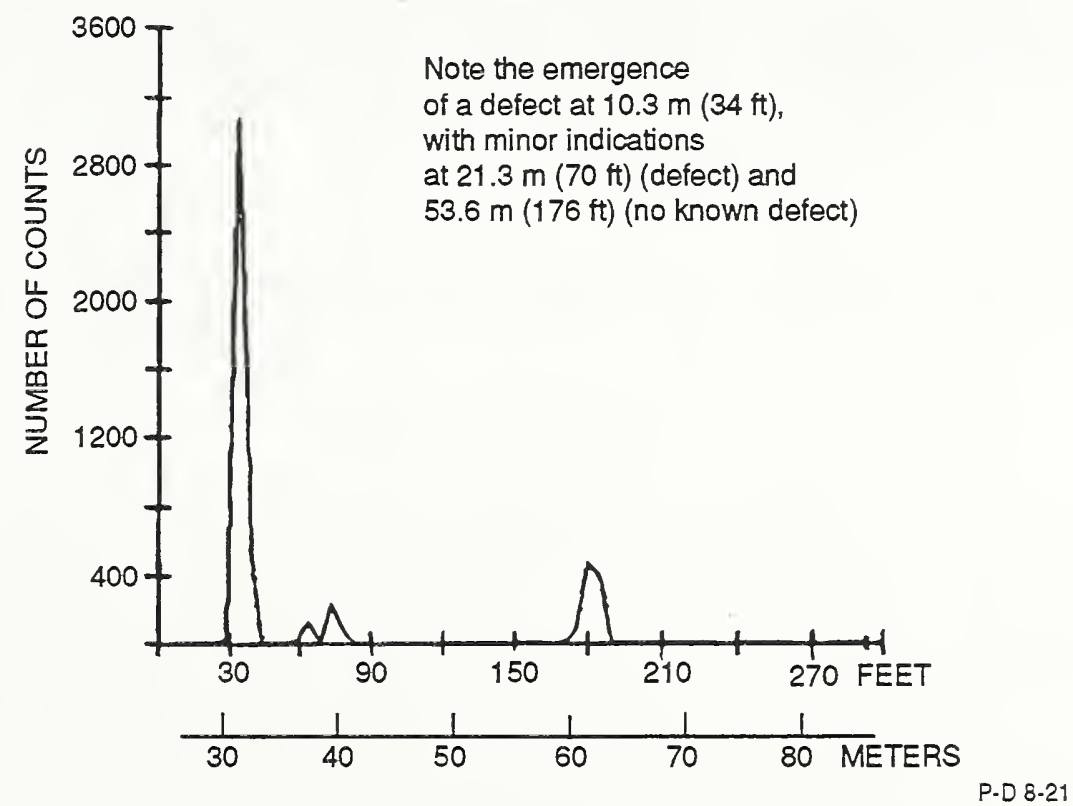

Figure 8-21. Histogram of time delays at $5.2 \mathrm{kV}$ and pruned data 


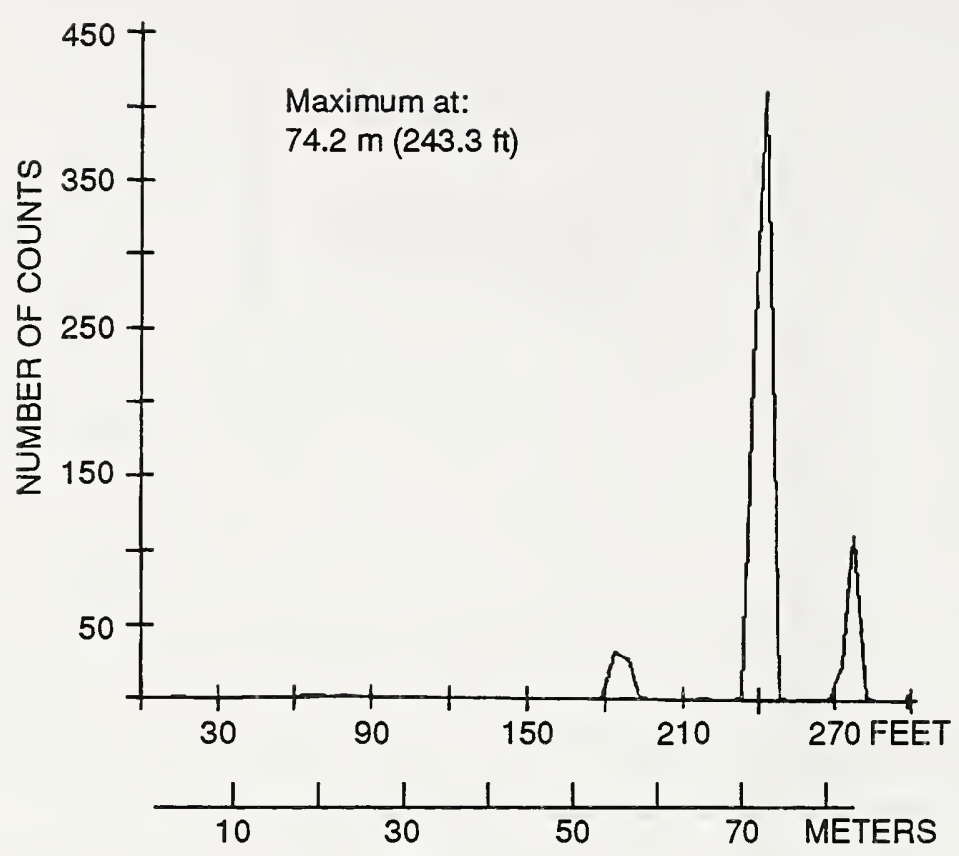

P-D 8-22

Figure 8-22. Histogram of time delays at $6 \mathrm{kV}$ and pruned data

three locations of 9.6, 21, and 72 meters. In addition, a fourth defect also appeared at 5.28 meters (176 feet). This defect was not intentionally introduced into the cable and did not appear in earlier tests. It is not clear whether this defect was innocuous, but it did show up during the final tests. This defect is shown in figure 8-23 and might be an example of a manufacturing defect that became worse because of the extended high electrical stress, considering that high voltage was applied to this cable for more than a total of 100 hours over the course of many trials. 


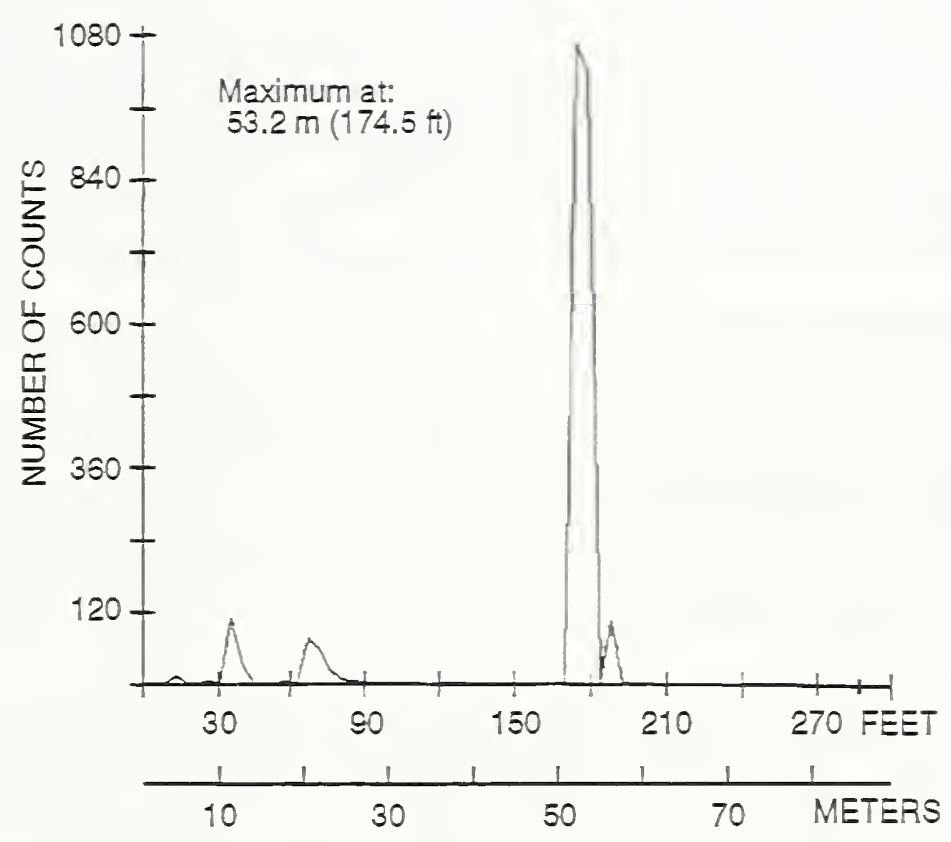

F.D $8-23$

Figure 8-23. Emerging defect in the test cable after an extended period of exposure (more than 100 hours) to high voltage 


\section{Discussion of Results}

\subsection{General}

The results described in the preceding section demonstrated that the method of partial discharge analysis (PDA), first developed for medium-voltage cables, can be successfully applied to low-voltage cables. The tests that were performed provided new insights on both the principles and the experimental aspects of detecting partial discharges in cables. The tests were performed in a laboratory environment, clearly a more benign environment than that of a power plant, so that some of the recognized concerns about noise filtering have yet to be addressed. The initial project plan envisioned a field trial of the method, but, as discussed in Section 9.3 below, the method did not gain sufficient acceptance among the electric utility industry within the time span of the project to make possible such a field trial. Thus, the method was proven to be applicable - the scope of the project - but also left a number of questions - beyond the scope of the project - unanswered. These questions have both technical and non-technical aspects.

\subsection{Technical aspects}

\subsubsection{Nature of the cables}

The results obtained during the last stages of the project clearly demonstrated the capability of the PDA method to detect defects in coaxial and multiconductor cables. For two-conductor cables, defects can also be found but might require further refinements in the test procedure to reveal all defects present in one cable. Tests were not performed on single-conductor (unshielded) cables because of the absence of a ground plane in such a case; however, a bundle of single cables might make it possible to detect defects, as illustrated by the case of the multiconductor experiment.

\subsubsection{Natural vs. artificial defects}

All the tests performed during this projects involved cables where artificial defects were inflicted on the test cable by mechanical damage to the insulation. Ideally, tests should be performed on cables obtained from the field in which natural defects might have developed. However, no such cables could be obtained. Conversely, if the behavior of the artificial defects created during this project could be compared to the behavior of natural defects (either occurring in the field or as the result of an accelerated aging program), some insight could be gained on the differences between 
the artificial mechanical damage and the natural defects. A proposal was made to make available one of the mechanically-damaged test cables to the Sandia National Laboratories where LOCA testing is performed; however, the logistics involved in performing such an a posteriori test made the proposal impractical.

\subsubsection{Practical methods for field tests}

The tests performed in the laboratory benefitted from a more benign environment than the environment that would be encountered in field tests. At this stage of the project, attention was focused on the major questions of principle rather than on "details" of practical application. It should be recognized, however, that some of these "details" might become major obstacles for field application. One example of such an unresolved question is the occurrence of partial discharges at the point of connection of the test equipment and at the far end of the cable. As described in Section 8.3.1, this problem was identified but set aside by the expedient of immersing the connection or termination in oil, clearly impractical for the field.

An even more serious potential technical problem in making the method acceptable is the need to disconnect any equipment at the far end of the cable. Understandably, power plant operators will not welcome the requirement of such a disconnection. Realistically, however, no electrical test method has been suggested as applicable to finding cable-only characteristics that does not require isolating the cable from connected equipment.

\subsubsection{Operator skill vs. expert system}

At this stage of the progress, the successful demonstration still relied on the skill and intuition of the operator when performing the data analysis. This situation is acknowledged, and a discussion of further developments that could reduce dependence on this skill is presented in several sections of this report. One should keep in mind that the scope of the project was to identify and assess existing methods, not to engage in research and development of new methods.

In view of the unique and highly specialized types of cable configurations that are likely to be encountered in nuclear power plants, one should question the advisability of using normal maintenance personnel in performing cable testing. It is unlikely in the forseeable future that PD measurement systems can be made sufficiently "smart" to eliminate the need for operation by specialists. With presently available technology, any use of partial discharge as a cable diagnostic must be viewed as a research and development project involving sophisticated instrumentation and computer analysis techniques. 


\subsubsection{Test voltage}

One of the initial expectations, namely that the inception voltage at a defect site would be lower than the PD inception voltage for a "sound" cable, was found incorrect. With hindsight, this expectation was not realistic. Thus, the question arises that the test procedure will involve test voltages above the inception voltage of a sound cable, giving rise to concerns about the possibility that such voltages might induce defects, or aggravate defects, in a cable that otherwise might still be in satisfactory condition. Such a concern is a leitmotif in any discussion of insulation test methods. One should not lose sight of the fact that the duration of application of the test voltage can be made considerably shorter than required for conventional high-voltage testing, thanks to further improvements in the speed of data acquisition that could be achieved with a dedicated hardware/software system, compared to the general-purpose system that was used in this project.

An unexpected result that holds great promise is the effect of applying the test voltage in a series of steps, producing a "turn-on" effect at various discharge sites that occurs for some voltage levels and does not occur at some other levels. Thus, by scanning a range of test voltages, it might be possible to effectively scan the range of types of defects contained in the cable, looking for all defects.

\subsubsection{Retrospective}

In retrospect, other methods would also have been well-suited for identifying the partial discharge sites. One method would have plotted the reduced data on threedimensional axes. One axis would have been position, another pulse height and the other the number of counts. This plot would have resulted in a display representing a pulse-height analyzer as a function of position.

Another method would have plotted only the mean value of the PD as a function of position. This method would have emphasized only the strongest partial discharge sites. Hence, one could collect both small and large pulses and the higher count rate of the smaller innocuous pulses would not obscure the low count rate of large pulses. One could add a third dimension, the excitation voltage, and observe the partial discharge sites "turn on." The three-parameter matrix of Table 8-1 presents this type of summary.

Other statistical measures could also be plotted versus position. It is likely that higher order statistical quantities would be good indicators of the type of partial discharge. It has been shown that different types of partial discharge behave differently (Van Brunt \& Kulkarni, 1990) when higher order statistics are compared. The difference in the partial discharge results from the nature in which they are generated. Innocuous PD sites are expected to occur primarily at interfaces between insulators or between insulators and conductors. Defects are presumed to arise from complete exposure of the conductor through a large air gap (for example, through a crack). This, undoubtedly, would be a fruitful approach. 


\section{Possible Further Developments}

The scope of the project, as mentioned earlier, was to assess existing methods, not to engage in research. However, in the course of the work, a number of possible further derelopment opportunities were identifed. Other research teams are also identifying areas of promising research. For the sake of completeness, these opportunities are briefly described in this section. Note that Section 6.5 already described possible enhancements to signal analysis methods in the context of the general discussion of partial discharge in cables. The following sections provide a summary of the state of the art (as of 1992), and describe possible refinements to existing defect location. and possible refinements to defect identification techniques.

\subsection{State-of-the-art}

The state-of-the-art can be riewed from two different perspectives: from the algorithmic point of riew, or from the technology point of riew. Current signal processing algorithms, data acquisition systems and high-speed computers are rery sophisticated and there is no doubt that a system could be assembled with the latest equipment if enough capital were arailable. However, for an instrument that is ultimately to be used in the field, a compromise must be made between function and cost.

From the practical point of riew, the main cost in implementing a test program using the partial discharge analysis (PDA) method is not the cost of the instrument but that of the manpower. To minimize the manpower costs, the instrument should be designed to operate with as little human interrention as possible, and at the fastest speed possible. Another incentive, previously cited, for high speed is the beneft of reducing the duration of exposure to the test voltage. The systems described in this report used technology dating to the mid- to late eighties, because it was then readily arailable. Continuing progress in technologr and decreasing hardware costs will make this approach feasible.

Alternately, a system could be built 'from the ground up' with the adrantage of a more compact, self-contained instrument that could be less expensive after writing off the development costs. However, the expected market for such an instrument is rather limited, so that the likelihood for a manufacturer to undertake the development is low, especially in riew of the present state of acceptance of the method.

Many instrument manufacturers use a building block approach because it minimizes both development resources and time. Building blocks can proride higher performance because of the mature state of their development, compared to custom designs. However, the complete instrument then includes features that may not be 
needed for the particular application, making it larger and less easy to transport to the site.

Comparing these two approaches, the building block approach is the only one making sense. The development efforts would be limited to the interfacing of existing systems and to the software development of algorithms. One such configuration could be based on a PC-based platform using a 486 Central Processing Unit (CPU), resulting in an approximate hundred-fold improvement in performance compared to that reported here. Results could then be obtained in real time. Data acquisition would be sequential, with as many channels as necessary, controlled by an IEEE 488 interface. The high-voltage power supply would be a small, portable, resonant type, controlled by the computer.

\subsection{Refinements to defect location techniques}

The present system software uses one of the simplest delay estimators to minimize the amount of computation. Even so, the analysis portion of the program required several hours to execute. With the accelerated computations made possible by the future system described in the preceding section, the door would be open to a wide variety of algorithms that can enhance performance. Many situations could arise, described in the following, that will necessitate implementing a more sophisticated system.

It is recognized that all the testing was performed in a laboratory with low electromagnetic interference. From the test results on the different specimens, it became clear that the partial discharge (PD) sensitivity required for this type of testing is not very high. This fact is an advantage because high levels of PD mean a higher signal-to-noise ratio. In the laboratory, this situation means that no steps were required to eliminate the noise effects, a subject discussed in Section 6. In the environment of a power plant, there will be high levels of noise and steps will be required to eliminate the noise.

As indicated in Section 6, there are different methods that can be applied to eliminate noise. If noise is present and no steps are taken to eliminate it, the delays used to identify the defect sites can have large errors. To avoid this uncertainty, the noisy waveforms should be preprocessed prior to analysis for location of the partial discharge. Depending on the complexity of the implementation chosen, different techniques could be used. One successful method is generalized correlation in which the received signal is prefiltered, using an optimum filter to eliminate the noise. If the noise is stationary, this technique works well, as demonstrated in Section 6. If the noise is nonstationary (the statistics vary with time), then a filtering scheme known as adaptative interference cancellation would be effective, assuming that the nonstationary noise varies slowly enough. This method would essentially measure the 
interfering noise and subtract it from the noisy signal in a manner similar to a bridge technique.

Another factor influencing the performance of the system is the attenuation in the cables. The cables used in the laboratory tests were relatively short and attenuation was only a minor factor. As the cable length increases, as might be the case for a power plant, the losses increase exponentially. The performance of the simple estimators degrades and it becomes necessary to use more sophisticated methods. One problem is that the blind spot increases in length. The other is that the dynamic range of the system is not great enough to detect the reflected PD signals. The best solution to these two problems is to use a cross-correlator, but that requires access to both ends of the cable. If only one end is available, then it would be necessary to extend the cable as discussed in Section 6 and use a matched filter method to increase the sensitivity of the measurement.

Distortion also plays a major role in the ability of the measurement system to determine the position of the partial discharge site along the cable. It is possible that unusual grounding arrangements could adversely affect the propagation of the PD pulse along the cable. This one aspect was not investigated during the laboratory tests. The advent of such a situation would also require more sophisticated techniques.

Highly distorted waveforms are difficult to deal with and completely satisfactory algorithms do not exist. Two basic approaches are possible: deconvolution, or modelbased estimation. In the case of deconvolution, the desired result is to undo the distortion. The distorted waveform is modeled as an undistorted waveform passing through a linear transformation that distorts it. There are different approaches to deconvolution, but the basic idea is to obtain a model of the distortion (often by measurement), then apply an inverse transformation to undo the distortion. However, there is a tradeoff; as more of the distortion is removed, more noise corrupts the estimate, limiting the amount of distortion that can effectively be removed. The other technique is to use a model-based approach such as the maximum likelihood proposed by (Knapp, 1990).

\subsection{Refinements to defect identification techniques}

\subsubsection{Theoretical modelling and discharge physics}

Even for the simplest electrode configurations, partial discharges are complex stochastic processes that are not readily predictable by present theoretical models. The complexity is a consequence of the factors that control discharge initiation and growth. Partial discharges generally occur in regions where the unperturbed electric 
field is highly nonuniform. Moreover, the local electric field is significantly modified during the development of the discharge because of the accumulation of space charge and/or surface charge. In fact, discharge-generated charge densities generally become sufficiently large to reduce field strengths below the levels sufficient to sustain the continuation of discharge growth.

The charges generated during a PD pulse also produce memory effects whereby the residual charges from preceding discharge events can significantly influence the initiation and growth of subsequent pulses. This has been shown to be the case for acgenerated PD on dielectric surfaces (Van Brunt \& Cernyar, 1991). An understanding of how PD phenomena are affected by the accumulation of surface charge in insulating materials requires an understanding of the charge in disposition and dissipation mechanisms. It is known that the surface charges produced by PDs often exhibit complex, fractal-like density distributions. A satisfactory model that can predict such fractal patterns does not yet exist.

The use of PD measurements to assess insulation performance and integrity would be served by a better understanding of the fundamental discharge processes. Although complete theoretical models with universal predictive capability may be out of reach due to the enormous complexity of the phenomenon, there are aspects of the physics that could, if better understood, provide a guide to the types of discharge behavior that are to be expected under different conditions encountered in practical insulation.

Calculations of the unperturbed electric-field distributions associated with particular types of point defects such as voids or cracks within solid dielectrics would provide an indication of the likelihood that PDs could be initiated at these defects for particular stress levels. More information is needed about the mechanisms of electron ejection from charged and uncharged surfaces likely to be encountered at defect sites. This is important because the rate of electron release from surfaces is often the primary mechanism that determines the probability of $\mathrm{PD}$ initiation at given level of electrical stress. Both secondary and primary electron release processes are also important in sustaining PD activity once it is initiated. A possible secondary electron release mechanism that could be significant in sustaining the discharge is the Auger-type quenching at metal surfaces of atomic or molecular species in the gas that have been excited during prior discharge activity to electronic metastable states. The quenching process with associated electron release may be enhanced significantly by the presence of the high electric field levels required for discharge growth. Although this process has been predicted from quantum theory, it has never been directly verified by experimental observations. The importance of this process in sustaining $\mathrm{PD}$ activity requires more attention.

A complete theory of partial discharge will require incorporation of memory effects. Such a theory must necessarily be a stochastic theory which would only be capable of predicting probabilities for the occurrences at any given time of discharge pulses with a given magnitudes. It is possible for memory to extend far back in time to many 
previous half-cycles of the applied voltage. This is, in part, due to the fact that times between PD events on successive half-cycles are on the order of milliseconds whereas the times required for dissipation of charge deposited on dielectric surfaces by PD activity are typically on the order of many minutes. Thus, the factors that affect the charging and discharging dynamics of dielectric surfaces must be understood in order to develop a complete model for the PD process. Surface charge dissipation rates, for example, are known to be affected by adsorbed water as well as carbon deposits.

Because of the importance of memory effects, there is reason to question the possibility of correlations between observed PD magnitude such as indicated by a mean pulse height and the physical size of a point defect in the insulation. Development of a more realistic theory of PD phenomena must go hand-in-hand with measurements of $\mathrm{PD}$ pulse characteristics under controlled conditions.

\subsubsection{Stochastic analysis}

It was noted previously in this report that PD phenomena are inherently stochastic processes in the sense that they can be represented by a set of time-ordered random variables. The mechanisms of $P D$ initiation and growth can only be described in probabilistic terms. Thus it can be expected, as observed, that the PD process will exhibit significant statistical variability in such characteristics as pulse amplitude, pulse shape, and time (or phase) of occurrence. The process is also inherently nonMarkovian, that is, its stochastic properties are significantly influenced by memory propagation.

As demonstrated by the data presented here, an analysis of the results from PD measurements can only provide a statistical description and, at best, suggest a most likely cause. The reliability of such analysis depends on knowledge about the physics of the PD process, which, as noted before, is generally inadequate. Presently used standard PD-measurement systems provide only limited information about the statistical properties of PDs. For example, conventional methods may give only an average value for PD magnitudes. More advanced systems are capable of determining PD pulse-height distributions. Systems are now being used for research purposes to provide phase-resolved data on PD amplitudes or occurrence rates. Consideration has been given by some groups to the use of data from the latter types of measurements to provide a signature for the type of $\mathrm{PD}$ process, for instance, whether it occurs at a metal-dielectric interface or in an occlusion within a solid dielectric. The reliability of such a signature analysis approach has yet to be demonstrated.

Even phase-resolved PD data may be of limited usefulness for nonstationary PD processes that seldom yield well-characterized, reproducible results. The difficulty of obtaining reproducible $P D$ pulse-height distribution data for rather simple discharge geometries, for instance, point-plane gaps, has been noted previously (Van Brunt, 1991). Because of the memory effects associated with the PD process, there can be a strong coupling between the amplitude of a PD pulse and its time (or phase) 
separation from earlier PD events. This means that pulse amplitude and pulse time (or phase) of occurrence are not independent random variables. Thus small changes in discharge initiation probability which may correspond to changes in electron release rates from surfaces are reflected in corresponding changes in the pulse-amplitude distribution. This presents an enormous obstacle in the interpretation of pulse-height distribution data and in defining patterns in phase-resolved data that might be useful for pattern recognition purposes.

With a recognition of this difficulty, research is underway in the development of more refined $\mathrm{PD}$ pulse-height analyzers that might yield results that are relatively immune to nonstationary behavior and would thus be more suitable in defining signatures that can be used for pattern recognition. The refined method allows for the measurement in real time of numerous phase-restricted conditional pulse-amplitude and time (or phase) of occurrence distributions. For example, it is possible with this system to measure the amplitude distribution of only those pulses that are separated from the previous pulse by a fixed time if the amplitude, of the previous pulse lies within a specified range. The data from conditional distribution indicate whether or not the pulse amplitude is correlated with the time separation from and/or the amplitude of the preceding PD pulse and will provide a measure of the strength of these correlations. The system can be configured to measure many different types of conditional distributions and allows for examination of phase-dependent correlations. It can, for example, be configured to determine the probability distributions of the amplitudes and phase-of-occurrence of the $n$th pulse $(n=1,2,3, \ldots)$ within a particular half-cycle conditioned on the amount of charge deposited by PDs on the dielectric surface during an earlier half-cycle.

By imposing restrictions on the pulse-amplitude and phase distributions, one is, in a sense, separating out the effects of the dependencies of one random variable on the other variables. Not only are the effects of memory assessed in these measurements, but also effectively removed. One thus has an opportunity to examine a pulseheight distribution which is more characteristic of the particular properties (field configuration or composition) of the defect site.

At the present time these advanced PD measurement systems (stochastic analyzers) are not commercially available and are still being developed as tools for basic research into the detailed stochastic behavior of PD phenomena. Because of the relatively low levels of current research in this area, it appears unlikely that such devices will be available in the foreseeable future for general purpose insulation testing.

An alternative to the use of an instrument to do stochastic analysis in real time is to perform such an analysis with computer software using digitally recorded data such as presented in this report. Given that one has recorded data on the phase and amplitudes of all successive PD events, an analysis via computer that can give conditional distributions is straightforward. However, up to this time there have been no known attempts to implement this software approach. There are serious limitations to the use of stored data to determine stochastic properties. In order to 
ensure that there are sufficient data to perform a reliable stochastic analysis, it may be necessary to use enormous data files that lie outside of the storage capacity of all but "main frame" type computers. Moreover, there are distinct advantages to performing stochastic analysis in real time as data are acquired. First of all, it overcomes the problem of having to store enormous quantities of data. Secondly, it is possible to determine immediately the extent to which the process is nonstationary; and thirdly, the measured distributions can be displayed immediately and continuously as the data are accumulated.

Of course, the hardware approach to stochastic analysis also has its limitations. It can become unreliable, for example, under the conditions where the data have a high noise content or where the spacing between pulses become so small that it is impossible to record all events or to resolve successive events. There are certain types of pulse-burst corona discharges that could not be examined reliably with the existing stochastic-analyzer systems because the spacings of pulses within the burst fall below the limits required to detect all pulses defined by the data acquisition electronics.

It can be argued that both the hardware and software approaches to stochastic analysis of partial discharge should be developed in parallel. In many respects these approaches can be considered as complementary. The weakness of one may often be compensated by the strengths of the other. In any case, there appears to be a necessity for a more refined stochastic analysis of PD data if there is to be any hope of using such data for pattern recognition purposes.

Stochastic analysis by itself, however, will not ensure reliable pattern recognition. The factors that affect the stochastic behavior of PDs need to be identified and better understood. To achieve this it will be necessary to amass and evaluate stochastic data for a wide range of conditions likely to be encountered in practical systems. Data are needed to test the dependencies on various parameters that define the condition of a defect site. These parameters should include: solid material composition, void or crack geometry, dielectric surface conductivity, and composition of gas within a void. Additionally, dependencies on applied voltage, frequency and gas pressure need to be considered. One should acquire a data base on PD stochastics for well defined conditions. This data base can serve as a guide in establishing a data base for specific types of high-voltage equipment, e.g., different types of cables. It will probably be necessary that each type of equipment has its own data base. This is simply because the differences in noise characteristics, PD detection sensitivity, geometrical configuration, etc. could invalidate meaningful comparisons among data from different types of equipment.

It should also be realized that the use of advanced $\mathrm{PD}$-measurement techniques discussed in this report is not trivial. The state-of-the-art in PD measurements is still rapidly advancing, and at present it can be argued that not enough is known to build "smart" PD-measurement systems that can reliably identify the nature of a defect from a PD pattern. This is an active area of research where measurements must still be performed by expert scientists and engineers. Although the new methods show 
promise, a much higher level of investment will be required if those methods are to find general applicability to practical insulation testing in the near term.

\subsubsection{Artificial enhancement of PDs.}

In making tests of insulation integrity, especially in cables, it is desirable, if possible, to artificially induce PD activity at suspected defect sites without applying excessive overvoltage. The probability for PD initiation can, under some conditions, be enhanced significantly by either exposing the insulation to high-energy radiation (ultra-violet or x-rays) or by introducing high-ionization-rate gases such as helium or other nonelectronegative rare gases.

In case of irradiation, the effect is to enhance the release of free electrons into the high-field gaseous region of a fault site and thereby increase the probability for PD initiation at any given voltage level. The use of this method is still considered to be experimental and has only recently been applied successfully to the testing of epoxy spacers used in compressed-gas insulated cables (Braun et al., 1991; Rizzetto et al., 1988). For thick insulation, the radiation must be sufficiently energetic to penetrate through the material. One must therefore be concerned about the possible damaging effects of the radiation in deciding on the exposure times and intensity levels.

The effect of introducing a high-ionization-rate gas is to lower the PD inception voltage. This method is also experimental and is not without its limitations. It will only be effective if the gas can efficiently diffuse into the defect site and displace a large fraction of the gas of higher inception level that was originally present. In the case of capsulated defect sites that are not exposed to the atmosphere, the times required for diffusion of gas into the defect site can be excessively long. This method is therefore likely to be useful only for defects that occur at or near the outer surfaces of an insulator. This is especially true in the case of cables.

It should also be realized that although it may be possible to induce PD inception at voltages below those at which they might occur under "normal" conditions, the effect of these "lower-voltage" PDs in causing further damage at the defect site need not be any less than would be caused by PDs at higher voltages. This is because PDinduced damage generally results from the energetic electrons (in excess of $10 \mathrm{eV}$ ) that are produced in the discharge, and the mean time-average electron energy at the PD site may be relatively insensitive to the applied voltage. In fact, at a given electric-field strength, the mean kinetic energy of electrons in a high-ionization-rate gas like helium can be significantly higher than in a lower ionization-rate gas like air. It must be remembered that helium not only has a higher ionization rate than air at a given field strength, it also has a higher ionization potential. Therefore, in order to cause ionization in helium, the mean energy of electrons should be higher than for air. Calculations and measurements have previously demonstrated that this is indeed the case. 


\section{Conclusions and Recommendations}

Detection of incipient defects by the method of partial discharge (PD) signal processing appears promising in principle, based on a the successful tests performed on three types of cables used in power plants as described on this report. However, further demonstration is needed to explore the limitations resulting from the present requirements for generating usable $\mathrm{PD}$ signals. Limitations and promising aspects are listed below.

\subsection{Limitations}

- The PD inception voltage for a cable containing a defect does not appear to be lower than the PD inception voltage associated with a sound cable. Thus, the test may require a relatively high test voltage, which may become objectionable.

- This relatively high voltage is most likely unacceptable for the equipment connected at the far end of the cable. Thus, applying the partial discharge test will in general require that the equipment be disconnected at the far end of the cable.

- The method based on time-delay estimation requires that the two peaks from the direct and reflected pulses be separated sufficiently for the software to recognize them. Thus, a blind zone would exist at the end of the cable where defects cannot be located. However this problem can be overcome if a matching cable extension is connected at the end of the cable, after it is disconnected from the equipment.

- In its present form, the connection between the test leads and the cable to be tested can be the site of substantial discharges that tend to drown out the discharges originating from defect sites. While in principle the signal processing should be able to discriminate these, they place an added burden on the system.

- Unshielded, single conductor cables may not be amenable to the detection of partial discharges resulting from an incipient defect. Likewise, a defect limited to one side of one conductor in a two-conductor cable, oriented away from the other conductor, has a low probability of detection. On the other hand, those defect sites that have the lowest probability of detection are also those that are the least likely to cause a cable failure.

- Noise background in practical in-situ conditions may make the measurements difficult with portable, field-type instrumentation.

- The ultimate goal of performing in-situ tests using staff maintenance personnel will require a substantial amount of signal processing and "expert system" 
development - an activity which was not included in the present scope of the project. Nevertheless, the promising aspects of this method deserve consideration, and are discussed in the following subsections.

\subsection{Promising aspects and further work needed}

It is clear from the results of the work presented here that it is possible to identify and locate "point defects" in cables by the detection of induced PD activity. With further work on development of stochastic analysis, it should be possible to perform more reliable pattern recognition for identifying the type of discharge site.

Present commercially available PD measurement systems are not reliable enough to provide positive identification of incipient faults in remote testing of cables. More work is needed in development of broad-band pulse detection, signal processing, and stochastic analysis of data. Considerably more work is needed to investigate the basic physics of PD phenomena in order to better understand the factors that determine the stochastic behavior of PDs under different conditions. Future research should include computer simulations of partial discharge that can be used in the testing and calibration of measurement systems.

\subsubsection{Technical work}

The work reported in this document demonstrates that partial discharge can be used as a diagnostic tool for low-voltage cables. The method is complicated and substantial work remains to be done if it is to be refined to the point where it can be translated into a practical instrument. Several key areas need to be addressed some areas are simple because solutions are readily available; other areas involve testing to determine the limitations of the method.

\subsubsection{Electromagnetic field analysis}

Study of the electromagnetic field configurations for the different cable constructions is an important aspect of this method. In one respect it is important to understand how the signals propagate along cables. In another respect it is important to understand what field stress is actually applied to the insulation system.

For the analysis algorithms to work properly the partial discharge pulse must have a certain fidelity. It is possible to correct for moderate distortions, however if the signal is too distorted, it is not possible to obtain reliable estimates of the location of the $\mathrm{PD}$ site. In extreme cases, it may not be possible to detect a pulse. 
The fidelity of the received PD pulse is intimately related to the propagation characteristics of the cable system under test. Utilities use different cable routing strategies, so it is a fair assessment that many varying structures exist. Not only are the routing structures different but the cable construction is not uniform throughout the industry. To understand the limitations of this method it will be necessary to make field measurements of the propagation characteristics of typical cable installations.

A uniform, conducting cable tray provides a reasonable ground plane. However, if the continuous nature of this tray is interrupted, then this will introduce an impedance discontinuity in the signal path. This is true eren if the cable tray is grounded via another connection. This is an important effect that needs study. Along with this effect, the influence of nearby grounded structures should be studied. Nearby grounded structures influence signal propagation because significant portions of the signal propagate in the region external to the insulation.

Partial discharge pulses see a relatively uniform propagation path as they travel along multiconductor cables. If a partial discharge occurs between the cable and the cable tray; a completely different path is taken. Initial indications suggest that when this alternate path is used, the received signal contains a telltale distortion that is readily identified. Further study of this case is needed so that partial discharges external to the cable can be distinguished from internal partial discharges.

Different types of insulations have different losses. One primary physical limitation of this method is the signal loss incurred during propagation from the defect site to the receiver. Also related to this limitation is the cable length since the signal attenuation is exponentially related to the cable length. This type of information is not readily available and must be measured. To specify the limitations of this method a data base of material properties will have to be developed.

Another critical issue is what criteria must the defect meet to assure its detection. When voltage is applied to the cable it is expected that partial discharge will start at a defect point. In the simplest case, where there are only two conductors, it is easy to see that the electric field is most intense in the region between the conductors, while the field is lowest in the region exterior to the cable. Eventually, as the roltage is increased the field on the exterior will become strong enough to cause partial discharge there also. While one can state that a PD inception voltage exists for these external defects, this does not guarantee detectability. As the inception voltage for this external defect is reached, other innocuous partial discharges mat obscure the defect. At this point it is not clear what defects are detectable and which are not.

Questions about the detectability could be answered using a two-prong approach. First, rigorous models should be developed for trpical multiconductor configurations. Numerical modeling will provide insight into the magnitude of the electric field at various positions within the cable. Also, this analysis would aid in selecting the 
best grounding strategy for the other conductors and nearby cables. Cracks due to embrittlement are small and irregular and would be nearly impossible to model on a computer. The second approach would encompass testing of various defects located at different positions within the cable. This step would provide the verification of the computer models used to assess electric field strength. Verification would be difficult because the experiment would have to designed to eliminate (or differentiate between) extraneous partial discharges. Furthermore, a realistic method for inducing defects into cables would need developing.

\subsubsection{Signal processing}

Partial discharge signals are very weak signals and their detection can be made difficult by the fact power plants present electrically harsh environments. In the experimental work of this report, interference was minimal. Some advanced noise reduction techniques exist that would significantly enhance the performance of the measurement in electrically harsh environments. One effective technique involves realtime measurement of the statistical characteristics of the interference and incorporates this information into an optimum filter to suppress the interference. Another method uses a second reference channel, perhaps an adjacent cable, to measure the interference. The interference in the cable under test and the reference cable are compared to determine their relationship. Once this relationship is determined the interference from the reference channel is filtered in an optimum manner so that it is as identical as possible to the interference in the cable under test. The filtered interference from the reference cable is then subtracted from the received signal in the cable under test. The result is that most of the interference is eliminated and only the PD pulse remains.

One other method that would be fruitful is referred to as deconvolution. As the partial discharge propagates along the cable under test it becomes distorted. This distortion limits the effectiveness of the PD method. If one knows the mathematical form that the distortion takes, then, in principle, the effects of the distortion can be undone. There are limits to this technique. Although it is usually impossible to predict the mathematical form of the distortion, it can often be measured. Real world signals are always noisy and the numerical representation is finite. Both of these limit the effectiveness of numerical deconvolution. The main distortion is a band-limiting effect and deconvolution can restore some of the lost bandwidth. In practice, bandwidth restoration is limited to factors of two to five before the ill-conditioned nature of the procedure introduces unacceptable levels of noise. 


\subsubsection{Blind spot elimination}

One of the limitations of the present measurement system is that there are blind spots in the cable. These blind spots are due to ambiguities in the determination of the PD location. If the PD site is located too close to the ends of the cable, it can be confused with partial discharges from the terminations of the cable or noise pulses that are coupled into the ends of the cable. A simple but effective method is available that eliminates these blind spots. By adding a piece of sound cable at the far end, the length of the cable is increased slightly, moving the blind spot into a dummy section from which no data are sought.

\subsubsection{Pulsed power supply}

The instrumentation system used continuous voltage to energize the cable. The data acquisition system collected data rapidly but transfer to the computer was very slow. The data collection period covers only a fraction of a second. Intermediate data transfer to the computer took several minutes with the voltage maintained at the cable terminals. Signal processing boards are now available that could economically increase the processing speed by two orders of magnitude.

\subsubsection{Cable termination}

One of the difficulties that surfaced during the investigation was the occurrence of partial discharge at the cable terminations. The activity was so significant that large portions of the collected data were not valid because they were due to the partial discharge at the termination. This places added burden on the data acquisition system as well as greater demands on the processing system. An elaborate scheme, using $\mathrm{SF}_{6}$ was used to reduce unwanted partial discharges. Immersing the terminations in oil also helped reduce the unwanted partial discharges. Neither of these methods is suitable for use in the field. Efforts should be directed at determining a simple, cost-effective method for eliminating partial discharges at the cable terminations.

\subsubsection{Defect identification}

During the investigation it was demonstrated that a highly trained indiridual could accurately interpret the results and correctly identify the positions of man-made defects. It is unrealistic to assume that the instrument operator would always be able to make reliable interpretation of $P D$ data. The instrument should include a expert-system data base to assist in the interpretation. 


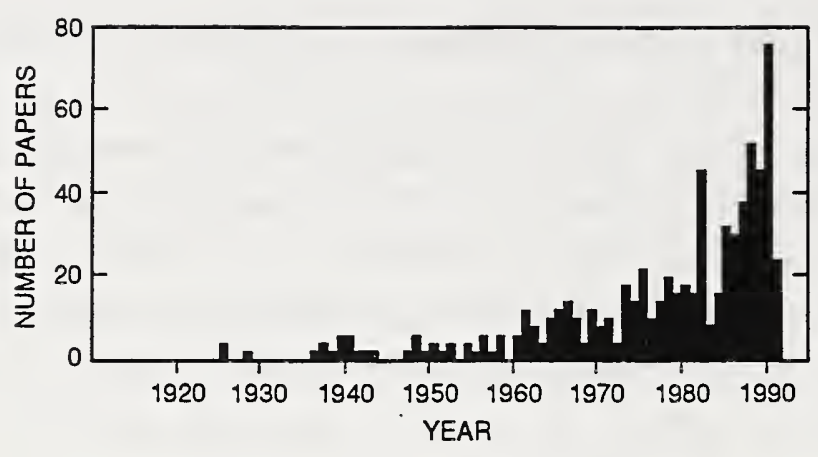

Figure 11-1. Growth of world-wide literature on partial discharge (source: Van Brunt, 1991)

\subsubsection{Acceptance of the method by utilities}

Some of the limitations of the method, described in Section 11.1, will unavoidably act as a deterrent for utilities to accept the validity of the method. However, these limitations should be balanced against the promising aspects of the method, which would gain more recognition if the additional research described under Section 11.4 could be undertaken.

As pointed out in the introduction, the detection of localized defects is only one of the possible tools that can be applied to one of the many possible types of incipient defects. Thus, the argument that some defects might not be detected by PD signal processing is not valid. A sustained awareness of the wide range of test methods will place each in perspective, which is the theme of the Nuclear Regulatory Commission (NRC) programs, of the related programs at the Electric Power Research Institute (EPRI), and of the work conducted in some U.S., Canadian, and other utilities.

\subsection{Recommendations for action}

The need for continuing research in the field of $\mathrm{PD}$ phenomena aimed at nuclear power plant aging studies should be considered in the context of current efforts in the general area of PD studies. It has already been stated that fundamental research is beyond the scope of the investigation covered in the present report; nevertheless, it is significant to note the near-exponential growth of general publications on this subject (figure 11-1), but still a lack of acceptance in the case of the nuclear power plant industry. The tasks identified in the following three subsections represent a challenging menu, both for the technical aspects of the research and for the allocation of resources. Notwithstanding the issue of resource allocation, the following recommendations are offered from the purely technical point of view. For convenience of presentation, they are separated in three categories, but of course they are interrelated and complementary. 


\subsubsection{Basics on partial discharge phenomena}

Little information is available on the nature of cable defects that are likely to be sites for $\mathrm{PD}$ formation. One of the reasons is the very success of industry in providing sound cable insulation. Nevertheless, cables removed from the field and presumed to contain incipient defects should be made available for laboratory tests in order to investigate the nature of partial discharge in such defects, as opposed to artificial defects. With some power plants reaching the stage of decommissioning, such cables might become more readily available.

Different cable geometries are stressed differently when a high test voltage is applied. Fundamental to the success of the PDA method is the understanding of the relationship between the applied test voltage and the resultant electric field applied across the dielectric. Experimental and numerical modeling would greatly aid in the understanding of this relationship and the level of applied field necessary to cause partial discharge.

The use of ionizing gases has been established as one means of reducing the voltage necessary to reach the PD inception, voltage. Technology is readily available for pumping gas down the conductors of insulated wires. This method is one avenue that could prove fruitful as an enhancement to the PDA method.

Once the PD pulse has been launched in the cable, the propagation characteristics of that cable will influence the waveform of the pulse arriving at the instrument. Hence, better understanding of the propagation characteristics is needed to take advantage of methods that include the waveform patterns in the analysis of the PD data.

The PDA method depends on the statistical nature of the discharges. Very little information is available on the statistical differences that may exist between the partial discharge related to incipient defects and that related to innocuous background.

\subsubsection{Hardware development}

One of the limitations of the PDA method as implemented during this project is the acquisition of a large number of irrelevant data that must then be eliminated by the software-driven analysis. Discrimination against these irrelevant data by hardware filtering before analysis should be investigated as a means to shrink the volume of data to be processed by the software.

The data acquisition hardware used in the experimental work of this project is antiquated, four years after initiation of the project and commitment to the hardware complement. Digitizing oscilloscopes have become available that can capture in 30 seconds data that previously took 20 minutes. Using such hardware would 
dramatically increase the amount of experimental work that could be performed, and the resultant understanding of the PD phenomena associated with incipient cable defects.

Practical methods are needed for attaching the test equipment to the cable under test in such a manner that no overpowering PD signals would be generated at the point of connection.

One of the primary goals of the instrumentation system should be to reduce the duration of exposure of the cable to the high voltage during the test. Using a power supply that can be energized under computer control would permit applying the voltage only during the time when actual data acquisition takes place. The high voltage would be turned off during data transfer and archiving procedures.

Testing with a conventional $60-\mathrm{Hz}$, corona-free transformer is cumbersome because of the size and weight of the equipment. Alternative methods should be investigated that eliminate bulky equipment.

\subsubsection{Software development}

Three tasks have been identified as potential or necessary enhancement to the PDA method as demonstrated during this project:

- Develop a generalized matched filter that would improve the PD pulse recognition for analysis after the attenuation along the cable.

- Develop a cross-correlation method that can be applied when both ends of the cable are available. With the likely necessity of disconnecting any equipment at the far end of the cable, this is a realistic opportunity.

- Last but not least, the success and eventual acceptance of the method demand that the equipment be operated by maintenance personnel available to the utilities, not by highly specialized experts on PD technology. Therefore, the expertise of the specialists need to be converted into a data base accessible to an expert system incorporated with the instrument that will assist the maintenance personnel in performing meaningful tests.

Implementation of a reliable partial-discharge measurement method for evaluating the integrity of cable insulation will require a major research effort. Studies of PD phenomena should be carried out not only using cables similar in construction and configuration to those actually installed in power plants, but also in well defined discharge gap geometries that simulate the types of faults that can occur. Techniques for complete stochastic analysis of PD pulse behavior now being developed and tested, need to be applied to the PD measurement process. 


\section{Acknowledgements}

The work reported here covered a period of four years and involved a multidisciplinary team at NIST, supported by the U.S. Nuclear Regulatory Commission Research Program, where W.S. Farmer and J.P. Vora provided guidance on the program objectives and review of the manuscript of this report.

Technical management at NIST was provided by W.E. Anderson, B.A. Bell, and O. Petersons.

The technical team, in addition to the authors, included D.R. Flach, E.F. Kelley; T.F. Leedy, M.E. Parker, and A.G. Perrey.

Graphics were contributed by R.J. Mele and R.H. Palm.

Organization and typesetting of the report were contributed by Mrs. R.J. Martucci. Additional review of the program was provided by $\mathrm{K} . \mathrm{N}$. Mathes and through the participants of the EPRI Workshops organized by G. Sliter. The draft of this report was reviewed by K.I. Stricklett. All these contributions are gratefully acknowledged. 


\section{References}

Ahmed, S.; Carfagno, S. P. and Toman, G. J. Inspection, Surveillance, and Monitoring of Electrical Equipment Inside Containment of Nuclear Power Plants With Applications to Electrical Cables, NUREG/CR-4257 ORNL/Sub/83-28915-1, Oak Ridge National Laboratory, Oak Ridge, TN, September 1985.

Anderson, W. E.; Ramboz, J. D. and Ondrejka, A. R. Final Report: Technical Contributions to the Development of Incipient Fault Detection/Location Instrumentation, NBSIR 86- 3392, National Bureau of Standards, Gaithersburg, MD, April 1986.

Anderson, W. E.; Ramboz, J. D. and Ondrejka, A. R. The Detection of Incipient Faults in Transmission Cables Using Time Domain Reflectometry Techniques: Technical Challenges, IEEE Transactions PAS-101, No. 7, July 1982.

Bammert, U. and Beyer, M. Partial Discharges Measured with an Automated System in Epoxy Resin and Polyethylene, IEEE Trans. Elec. Insul., Vol. 23, pp. 215-225, 1988.

Barclay, A. L.; Sweeney, P. J.; Dissado, L. A. and Stevens, G. C. Stochastic Modelling of Electrical Treeing: Fractal and Statistical Characteristics, J. Phys. D: Appl. Phys., Vol. 23, pp. 1536-1545, 1990.

Bartnikas, R. A Commentary on Partial Discharge Measurement and Detection, IEEE Transactions EI-22, pp. 629-653, 1987.

Bartnikas, R. Detection of Partial Discharges (Corona) in Electrical Apparatus, IEEE Transactions EI-25, pp. 111-123, 1990.

Beyer, M.; Kamm, W.; Borsi, H. and Feser, K. New Method for Detection and Location of Distributed Partial Discharges (Cable Faults) in High Voltage Cables Under External Interference, IEEE Transactions PAS-101, pp. 3421-3438, No. 9, Sept., 1982.

Boggs, S. A. Partial Discharge - Part I: Overview and Signal Generation, IEEE Electrical Insulation Magazine, Vol. 6, pp. 33-39, 1990.

Boggs, S. A. and Stone, G. C. Fundamental Limits in the Measurement and Detection of Corona and Partial Discharge, IEEE Transactions EI-17, pp. 143-146, 1982.

Brancato, E. L. Insulation Aging: A Historical and Critical Review, IEEE Transactions EI-13, No. 4, August 1978.

Braun, J. M.; Rizzetto, S.; Fujimoto, N. and Ford, G. L. Modulation of Partial Discharge Activity in GIS Insulators by X-ray Irradiation, IEEE Transactions on Electrical Insulation, Vol. 26, pp. 460-468, 1991. 
Bulinski, A. T.; Bamji, S. S. and Densley, R. J. Factors Affecting the Transition from a Water Tree to an Electrical Tree, Conf. Record-1988 Int. Symp. Elec. Insul., IEEE publication CH 2594-0/88, pp. 327-334, 1988.

Bustard, L. D. Definition of Data Base, Code, and Technologies for Cable Life Extension, SAND86-1897UC-78, Sandia National Laboratories, Albuquerque, NM, March 1987.

Clough, R. L. and Gillen, K. T. Investigation of Cable Deterioration Inside Reactor Containment, Nuclear Technology, Vol. 59, November 1982.

Crichton, G. C.; Karlsson, P. W. and Pedersen, A. A Theoretical Derivation of the Transients Related to Partial Discharges in Ellipsoidal Voids, Conference Record of the 1988 IEEE Int. Symp. on Elec. Insul., Boston, MA, IEEE publication CH25940/88, pp. 238-245, 1988.

Crichton, G. C.; Karlsson, P. W. and Pedersen, A. Partial Discharges in Ellipsoidal and Spherical Voids, IEEE Transactions EI-24, pp. 335-342, 1989.

Davis, J. C. The Physics of Partial Discharges in Solid Dielectrics, IEEE Transactions EI-19, pp. 475-495, 1984.

Dinsel, M. R.; Donaldson, M. R. and Soberano, F. T. In Situ Testing of the Shippingport Atomic Power Station Electric al Circuits (Draft), NUREG/CR-3956 EGG-2443 (DRAFT), EG\&G Idaho, Inc., Idaho Falls, ID, March 1986.

Dissado, L. A. Characterization of the Growth of Water Trees, IEEE Transactions EI-17, pp. 392-398, 1982.

Dissado, L. A. Theoretical Basis for the Statistics of Dielectric Breakdown, J. Phys. D: Appl. Phys., Vol. 23, pp. 1582-1591, 1990.

Dissado, L. A. and Hill, R. M. The Statistics of Electrical Tree Inception, IEEE Transactions EI-25, pp. 660-666, 1990.

Egli, W. and Eliasson, B. Numerical Calculation of Electrical Breakdown in Oxygen in a Dielectric-Barrier Discharge, Helvetica Physica Acta, Vol. 69, pp. 302$305,1989$.

Eliasson, B. and Kogelschatz, V. Modeling and Applications of Silent Discharge Plasmas, IEEE Trans. Plasma Science, Special Issue, April 1991.

EPRI Workshop, reported in Proceedings: Workshop on Power Plant Cable Condition Monitoring, EPRI EL/NP/CS/-5914-SP, July 1988.

Franks, L. E. Signal Theory, Chapter 9 Dowden and Culver, Stroudsburg, Pa., 1981.

Gardner, J. B. and Shook, T. A. Status and Prospective Application of Methodologies from an EPRI Sponsored Indenter Test Project, Proceedings: Workshop on Power Plant Cable Condition Monitoring, EPRI EL/NP/CS/-5914SR, July 1988. 
Gardner, J. B. and Winslow, J. W. Aging and Test Standards Related to Cable Condition Monitoring, Proceedings: Workshop on Power Plant Cable Condition Monitoring, EPRI EL/NP/CS/-5914- SR, July 1988.

Geymer, D. O. Polypropylene in The Radiation Chemistry of Macromolecules Vol. 2, M. Dole ed., Academic Press, New York, 1973.

Goamez-Garcia, M.; Bartnikas, R. and Wertheimer M. R. Modification of XLPE Exposed to Partial Discharges at Elevated Temperature, IEEE Transactions EI-25, pp. 688-692, 1990.

Gradin, L. P. Evolutionary Development of Industry Standards Related to Cable and Present Standards Activities for Cable Condition Serviceability Determination, Proceedings: Workshop on Power Plant Cable Condition Monitoring, EPRI $E L / N P / C S /-5914-S R$, July 1988.

Gulski, E. and Kreuger, F. H. Computer-aided Analysis of Discharge Patterns, J. Phys. D: Appl. Phys., Vol. 23, pp. 1569-1575, 1990.

Hedwig, P. Electrical Conductivity of Irradiated Polymers in The Radiation Chemistry of Macromolecules Vol. 1 M. Dole ed., Academic Press, New York, 1972.

Hero, A. and Schwartz, S. A New Generalized Cross-Correlator, IEEE Transactions ASSP-33, pp. 38-45, No. 1, Feb., 1985.

Hikita, M.; Yamada, K.; Nakkamura, A.; Mizutani, T.; Oohasi, A. and Jeda, M. Measurements of Partial Discharges by Computer and Analysis of Partial Discharge Distribution by the Monte Carlo Method, IEEE Transactions EI-25, pp. 453-467, 1990.

Huzumi, N.; Okamoto, T. and Fukagawa, H. Simultaneous Measurement of Microscopic Image and Discharge Pulses at the Moment of Electrical Tree Initiation, Japanese J. Appl. Phys., Vol. 27, No. 4, 1988.

Jacobs, P. T. An Evaluation of Information Sources and Requirements for Nuclear Plant-Aging Research With Life-Extension Implications, IEEE Transactions NS-34, No. 1, February 1987.

Jaworska, E. and Kaluska, I. The Influence of the Dose Rate on Polyethylene Cross-Linking in the Presence of Additives, Proceedings of the Fifth Tihany Symposium on Radiation Chemistry, Vol. 2, 1095, 1983.

Jeppeson, D. Equipment Qualification Testing Methodology Sandia Laboratories, pp. 305-316, NUREG/CP-0041 Vol. 5, U. S. Nuclear Regulatory Commission, NBS, Gaithersburg, MD, October 1982.

Kelley, E. F.; Nehmadi, M.; Hebner, R. E.; Pace, M. O.; Wintenberg, A. L.; Blalock, T. V. and Foust, J. V. Measurement of Partial Discharges in Hexane Under DC Voltage, IEEE Transactions EI-24, pp. 1109-1119, 1989. 
Knapp, C. H.; Bansal, R.; Mashikian, M. S. and Northrop, R. B. Signal Processing Techniques for Partial Discharge Site Location in Shielded Cables, IEEE Transactions PWRD, 5, No. 2, April 1990.

Kreuger, F. H. Discharge Detection in High Voltage Equipment, American Elsevier, New York, 1965.

Kulkarni, S. V.; Van Brunt, R. J. and Lakdawala, V. K. Transition from Trichel-Pulse Corona to Dielectric Barrier Discharge, 1990 Annual Report Conference on Electrical Insulation and Dielectric Phenomena, IEEE, New York, pp. $267-274,1990$.

Kwaaitaal, Th. and van den Eijnden W. M. M. M., Dielectric Loss Measurement as a Tool to Determine Electrical Aging of Extruded Polymeric Insulated Power Cables, IEEE Transactions EI-22, No. 1, February 1987.

Lee. R. H. New Developments in Cable Systems Testing, IEEE Transactions IA-13, No. 3, May/June 1977.

Legler, W. The Statistics of Electron Avalanches in Electronegative Gases at High Field Strengths and for Large Gas-Amplification, Zeitshrift fur Naturforschung, Vol. 16A, pp. 253-261, 1961.

Malik, N. H. and Alrainy, A. A. Statistical Variation of DC Corona Pulse Amplitudes in Point-to-Plane Air Gaps, IEEE Transactions EI-22, pp. 825-829, December 1987.

Mandelkern, L. Radiation Chemistry of Linear Polyethylene in The Radiation Chemistry of Macromolecules Vol. 1, M. Dole ed., Academic Press, New York, 1972.

Margulis, L. A.; Gorelick, B. A.; Semenenko, E. I. Some Aspects of the Oxidation of PVC under Irradiation, Proceedings of the Fifth Tihany Symposium on Radiation Chemistry, Vol. 2, 875, 1983.

Mashikian, M.S.; Bansal, R. and Northrop, R. B. Location and Characterization of Partial Discharge Sites in Shielded Power Cables, IEEE Transactions PWRD 5, No. 2, April 1990.

Mashikian, M. S.; Northrop, R. B.; Bansad, Rajcev, and Nikas, C. L. Method and Instrumentation for the Detection, Location and Characterization of Partial Discharges and Faults in Electric Power Cables, U. S. Patent 4887, 041 , December 12, 1989.

Mason, J. H. Discharges, IEEE Transactions EI-13, pp. 211-238, 1978.

Mathes, K. N. Electrical Properties: Relationship to Evaluation and Performance of Cable Insulation, Notes for Power Cable Engineering Clinic, University of Wisconsin, 1987.

Meininger, R. D. and Dinsel, M. R. Passive Surveillance: A Technique to Characterize the Condition of Power and Control Circuits in a Nuclear Plant, Idaho National Engineering Laboratory, Idaho Falls, ID, 1985. 
Mopsik, F. I. The Use of Time-Domain Dielectrics Spectroscopy to Evaluate the Lifetime of Nuclear Power Plant Cables, NISTIR 4486, 1992

Mopsik, F. I. Precision Time-Domain Dielectric Spectrometer, Review of Science Instrum., Vol. 55, No. 1, January 1984.

Morrow, R. Theory of Negative Corona in Oxygen, Phys. Rev. A, Vol. 32, pp. 1799-1809, 1985.

Okamoto, T. and Tanaka T. Prediction of Treeing Breakdown from Pulse Height of Partial Discharge on Voltage-Phase Angle, Japanese J. Appl. Phys., Vol. 24, pp. 156-160, 1985.

Pedersen, A. Partial Discharges in Voids in Solid Dielectrics. An Alternative Approach, 1987 Annual Report - Conference on Electrical Insulation and Dielectric Phenomena, IEEE Publication 87 CH2462-0, pp. 58-64, 1987.

Petty, K. A. Application, Construction, and Testing of Generating Station Cables, EPRI EL/NP/CS-5914-SR, Proceedings: Workshop on Power Plant Cable Condition Monitoring, EPRI EL/NP/CS-5914-SR, July 1988.

Phillips, P. J. Characterization of Cable Aging, Proceedings: Workshop on Power Plant Cable Condition Monitoring, EPRI EL/NP/CS-5914-SR, July 1988.

Rizzetto, R.; Fujimoto, N. and Stone, G. C. A System for the Detection and Location of Partial Discharge using X-Rays, Conference Record - 1988 IEEE Int. Symp. on Elect. Insul., IEEE publ. CH-2594-0/88, pp. 262-266, 1988.

Salovey, R. Poly(vinyl Chloride), in The Radiation Chemistry of Macromolecules Vol. 2 M. Dole ed., Academic Press, New York, 1973.

Schifani, R. A Novel Histogram for Partial Discharge Signals in HV Insulating Systems, IEEE Trans. Elec. Insul., Vol. EI-21, pp. 89-99, 1986.

Schmidt, R. O. A Signal Subspace Approach to Multiple Emitter Location and Spectral Estimation, Ph.D. Thesis, Stanford Univ., Stanford, Calif., Nov., 1981.

Scott, D. A. and Haddad, G. N. Negative Corona in Nitrogen - Oxygen Mixtures, J. Phys. D: Appl. Phys., Vol. 20, pp. 1039-1044, 1987.

St. Onge, R. J. Cable and Electrical Apparatus Monitoring Program at San Onofre Nuclear Generating Station (SONGS) Unit 1, Proceedings: Workshop on Power Plant Cable Condition Monitoring, EPRI EL/NP/CS-5914-SR, July 1988.

Steiner, J. P. Digital Measurement of Partial Discharge, Ph.D. Thesis, Purdue University, School of Elec. Eng., West Lafayette, Ind., Chapters 3-4, May, 1988.

Steiner, J. P. Partial Discharge - Part IV: Commercial PD Testing, IEEE Electrical Insulation Magazine, Vol. 7, pp. 20-33, 1991. 
Steiner, J. P. and Martzloff, F. D. Partial Discharges in Low-Voltage Cables, Conf. Rec. 1990 IEEE Int. Symp. on Elec. Insul., Toronto, Canada, IEEE publication 90-CH2727-6, pp. 149-152, 1990.

Steiner, J. P. and Weeks, W. L. Digital Estimation of Partial Discharge, CEIDP Annual Report, (IEEE Conf. Record 87CH2462:0), Gaithersburg, MD, pp. 73-78, 1987.

Steiner, J. P.; Weeks, W. L. and Furgason, E. S. Robust Deconvolution of Correlation Functions, IEEE Ultrasonics Symposium, Denver, Colo., 1987.

Stone, G. C.; Sawyer, D. M. and Gupta, B. K. Electrical Testing of Generator Station Cables, Proceedings: Workshop on Power Plant Cable Condition Monitoring, EPRI EL/NP/CS-5914-SR, July 1988.

Stonkus, D. J. Physical Degradation Assessment of Generator Station Cables, Proceedings: Workshop on Power Plant Cable Condition Monitoring, EPRI $E L / N P / C S-5914-S R$, July 1988.

Tanaka, T. Internal Partial Discharge and Material Degradation, IEEE Trans. Elec. Insul., Vol. EI-21, pp. 899-905, 1986.

Toman, G. J. and Gardner, J. B. Development of Nondestructive CableInsulation Tests, Proceedings: ANS Topical Meeting on Operability of Nuclear Power Systems, 1986.

Van Brunt, R. J. Water Vapor-Enhanced Electron Avalanche Growth in $\mathrm{SF}_{6}$ for Nonuniform Fields, J. Appl. Phys., Vol. 59, pp. 2314-2323, 1986.

Van Brunt, R. J. Common Parameterizations of Electron Transport, Collision Cross Section, and Dielectric Strength Data for Binary Gas Mixtures, J. Appl. Phys., Vol. 61, pp. 1773-1787, 1987.

Van Brunt, R. J. Stochastic Properties of Partial-Discharge Phenomena: A Review, IEEE Transactions EI-26, October 1991.

Van Brunt, R. J. and Cernyar, E. W. Influence of Memory Propagation on Phase-Resolved Stochastic Behavior of AC-Generated Partial Discharges, Appl. Phys. Lett., Vol. 58, pp. 2628-2630, 1991.

Van Brunt, R. J. and Kulkarni, S. V. Method for Measuring Stochastic Properties of Corona and Partial Discharge Pulses. Rev. Sci. Instrum., Vol. 60, No. 9, pp. 3012-3023, September 1989.

Van Brunt, R. J. and Kulkarni, S. V. Stochastic Properties of Trichel-Pulse Corona: A Non-Markovian Random Point Process, Phys. Rev. A, Vol. 42, pp. 49084932, 1990.

Van Brunt, R. J. and Leep, D. Characterization of Point-Plane Corona Pulses in $\mathrm{SF}_{6}, J$. Appl. Phys., Vol. 52, pp. 6588-6600, 1981. 
Van Brunt, R. J.; Misakian, M.; Kulkarni, S. V. and Lakdawala, V. K. Influence of a Dielectric Barrier on the Stochastic Behavior of Trichel-Pulse Corona, IEEE Trans. Elec. Insul. (submitted, 1991).

Van Brunt, R. J.; Stricklett, K. L.; Steiner, J. P.; and Kulkarni, S. V. Recent Advances in Partial Discharge Measurement Capabilities at NIST, IEEE Trans. Elec. Insul. Vol. 27, pp. 114-129, 1992.

Van Trees, H. L. Detection, Estimation and Modulation Theory, Part pp. 309, Wiley, New York, 1968.

Vora, J. P. Nuclear Plant Aging Research - An Overview (Electrical and Mechanical Components, Office of Nuclear Regulatory Research - U.S. Nuclear Regulatory Commission), 1986.

Weeks, W. L. Electromagnetic Theory for Engineering Applications John Wiley and Sons, Inc., New York, 1964.

Weeks, W. I. and Steiner, J. P. Instrumentation for the Detection and Location of Incipient Faults on Power Cables, IEEE Transactions PAS-101, pp. 2328-2335, No. 7, July, 1982.

Weeks, W. L. and Steiner, J. P., Improvement in the Instrumentation for Partial Discharge Location in Cables, IEEE Transactions PAS-104, No. 4, April, 1985.

Wilson, J. E. Radiation Chemistry of Monomers, Polymers, and Plastics, Marcel Dekker, Inc., New York, 1974.

Proceedings: Workshop on Power Plant Cable Condition Monitoring, EPRI $E L / N P / C S /-5914-S R$, July 1988. 
NIST-114A

(REV. 3-90)
U.S. DEPARTMENT OF COMMERCE NATIONAL INSTITUTE OF STANDARDS AND TECHNOLOGY

\section{BIBLIOGRAPHIC DATA SHEET}

1. PUBLICATIOH OR REPORT NUMBER
NISTER 4487

2. PERFORMIHG ORGAMIZATIOH REPORT HUMBER

3. PUBUCATION DATE

JULY 1992

4. TITLEAND SUBTTILE

Detection of Incipient Defects in Cables by Partial Discharge Signal Analysis

5. AUTHOR(S)

F.D. Martzloff, E. Simmon, J.P. Steiner, R.J. Van Brunt

6. PERFORMINO ORGAMIZATION (IF JOINT OR OTHER THAN NIST, SEE INSTRUCTIONS) U.S. DEPARTMENT OF COMHERCE

MATIONAL INSTITUTE OF STANDARDS AND TECHNOLOGY

CANTHERSBURG, MD 20899

9. SPONSORINQ ORGAMIZATIOH MAME AND COMPLETE ADDRESS (STREET, CITY, STATE, ZIP)

U.S. Nuclear Regulatory Commission

Washington, DC 20555

10. SUPPLEMENTARY HOTES

11. ABSTRACT (A 2OO-WORD OR LESS FACTUAL SUMMARY OF MOST SIGNIFICANT INFORMATION. IF DOCUMENT INCLUDES A SIGNIFICANT BIBLUOGRAPHY OR UTERATURE SURVEY, MENTION IT HERE)

As one of the objectives of a program aimed at assessing test methods for in-situ detection of incipient defects in cables due to aging, a laboratory test system was implemented to demonstrate that the partial discharge analysis method can be successfully applied to low-voltage cables. Previous investigations generally involved cables rated $5 \mathrm{kV}$ or higher, while the objective of this program focused on the lower voltages associated with the safety systems of nuclear power plants.

The defect detection system implemented for this project was based on commercially available signal analysis hardware and software packages, customized for the specific purposes of the project. The test specimens included several cables of the type found in nuclear power plants, including artificial defects at various points of the cable.

The results indicate that indeed, partial discharge analysis is capable of detecting incipient defects in low-voltage cables. There are, however, some-iimitatiogs-ofitechnical and non-technical nature that need further exploration before this method can be accepted in the industry.

12 KEY WORDS (6 TO 12 ENTRIES; ALPHABETICAL ORDER; CAPTTALIZE ONLY PROPER MAMES; AND SEPARATE KEY WORDS BY SEMICOLONS) cable defects; LOCA; ĩuclear power plant; numerical analysis; partial discharge; time-domain spectroscopy

14. NUMBER OF PRIHTED PAGES

144

15. PRICE 

